\title{
Embryo culture media in human IVF
}

Citation for published version (APA):

Kleijkers, S. H. M. (2016). Embryo culture media in human IVF: effects on the offspring. [Doctoral Thesis, Maastricht University]. Datawyse / Universitaire Pers Maastricht. https://doi.org/10.26481/dis.20161027sk

Document status and date:

Published: 01/01/2016

DOI:

10.26481/dis.20161027sk

Document Version:

Publisher's PDF, also known as Version of record

\section{Please check the document version of this publication:}

- A submitted manuscript is the version of the article upon submission and before peer-review. There can be important differences between the submitted version and the official published version of record.

People interested in the research are advised to contact the author for the final version of the publication, or visit the DOI to the publisher's website.

- The final author version and the galley proof are versions of the publication after peer review.

- The final published version features the final layout of the paper including the volume, issue and page numbers.

Link to publication

\footnotetext{
General rights rights.

- You may freely distribute the URL identifying the publication in the public portal. please follow below link for the End User Agreement:

www.umlib.nl/taverne-license

Take down policy

If you believe that this document breaches copyright please contact us at:

repository@maastrichtuniversity.nl

providing details and we will investigate your claim.
}

Copyright and moral rights for the publications made accessible in the public portal are retained by the authors and/or other copyright owners and it is a condition of accessing publications that users recognise and abide by the legal requirements associated with these

- Users may download and print one copy of any publication from the public portal for the purpose of private study or research.

- You may not further distribute the material or use it for any profit-making activity or commercial gain

If the publication is distributed under the terms of Article $25 \mathrm{fa}$ of the Dutch Copyright Act, indicated by the "Taverne" license above, 


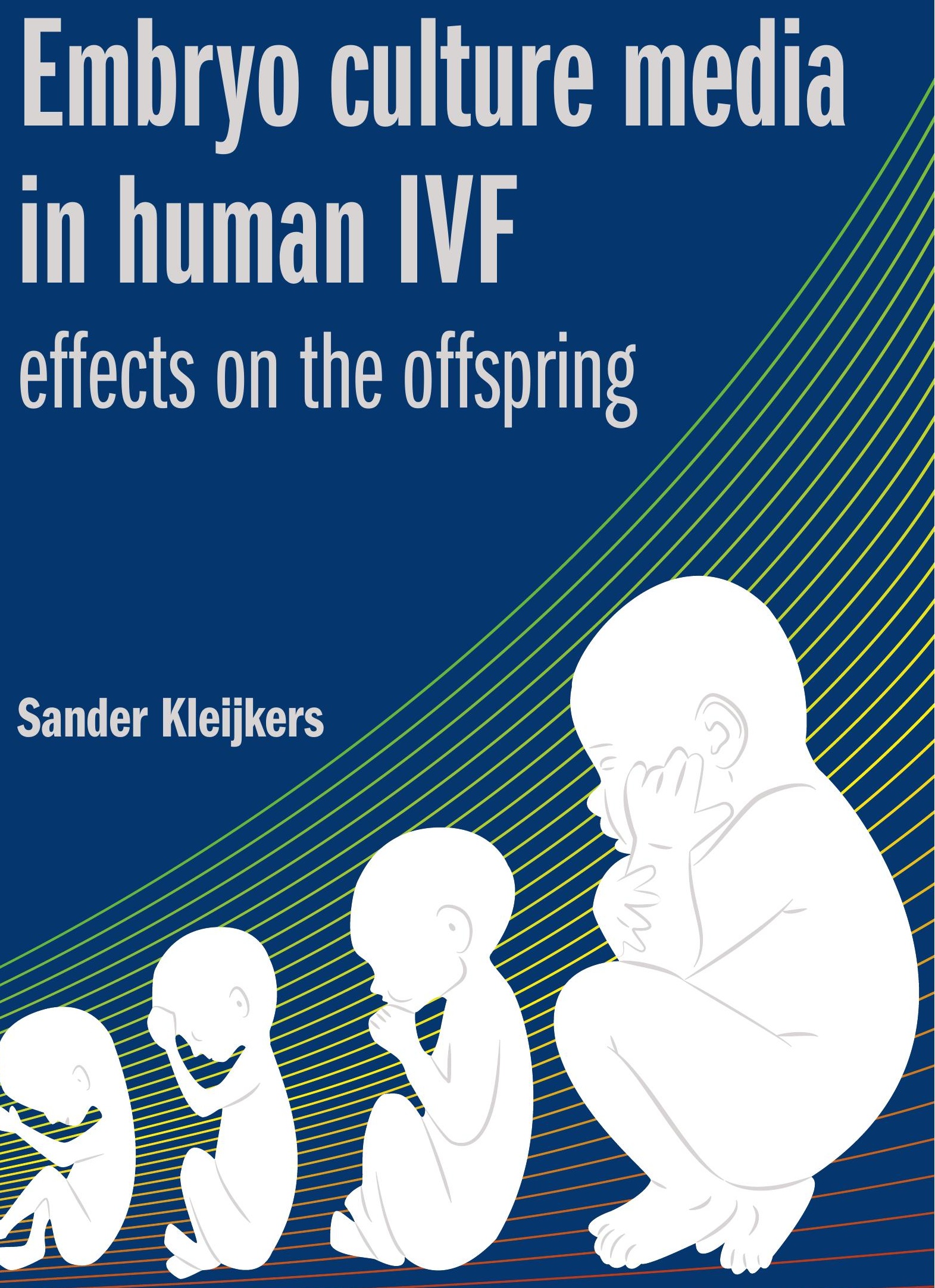


Copyright (C) 2016 Sander H.M. Kleijkers

Printed by: Datawyse | Universitaire Pers Maastricht ISBN 9789461596024 


\title{
Embryo culture media in human IVF effects on the offspring
}

\author{
PROEFSCHRIFT
}

ter verkrijging van de graad van doctor aan de Universiteit Maastricht, op gezag van de Rector Magnificus, Prof. dr. Rianne M. Letschert, volgens het besluit van het College van Decanen,

in het openbaar te verdedigen

op donderdag 27 oktober 2016 om 16.00 uur

door

Sander Hubert Mathijs Kleijkers 


\section{Promotor}

Prof. dr. J.L.H. Evers

\section{Copromotores}

Dr. J.C.M. Dumoulin

Dr. A.P.A. van Montfoort

\section{Beoordelingscommissie}

Prof. dr. M.E.A. Spaanderman (voorzitter)

Prof. dr. H.G. Brunner

Prof. dr. T.P. Fleming (University of Southampton, Verenigd Koninkrijk)

Prof. dr. E. van den Abbeel (Universiteit Gent, België)

Prof. dr. L.J.I. Zimmermann 


\section{Contents}

Chapter 1 General introduction and outline of the thesis

Chapter 2 Influence of embryo culture medium (G5 and HTF) on pregnancy and perinatal outcome after in vitro fertilization: a multicenter randomized controlled trial

Chapter 3 IVF culture medium affects postnatal weight in humans during the first 2 years of life

Chapter 4 Age of G-1 PLUS v5 embryo culture medium is inversely associated with birth weight of the newborn

Chapter 5 Ammonium accumulation in commercially available embryo culture media and protein supplements during storage at $2-8^{\circ} \mathrm{C}$ and during incubation at $37^{\circ} \mathrm{C}$

Chapter 6 Differences in gene expression profiles between human preimplantation embryos cultured in two different IVF culture media

Chapter 7 General discussion and future prospects 101

References 109

Valorization

Summary

Samenvatting

Dankwoord

Curriculum Vitae 



\section{CHAPTER 1}

General introduction and outline of the thesis 


\section{General introduction}

Subfertility has been described as the inability to achieve a pregnancy in spite of regular intercourse aimed at conception for more than 12 months (Evers, 2002). The estimated prevalence of subfertility is around $9 \%$ worldwide for women aged 20 to 44 (Boivin et al., 2007). Since the birth of the first child conceived by in vitro fertilization (IVF) in 1978 (Steptoe and Edwards, 1978), the use of assisted reproduction technologies (ART) has increased significantly. In the year 2011, a total of 761,896 ART cycles have been reported in Europe and the United States according to the European and American ART registries (Centers for Disease Control and Prevention, 2013; Kupka et al., 2016). In that same year, in Europe, the percentage of newborns conceived with in vitro techniques ranged from $0.2 \%$ in Romania to $5.8 \%$ in Denmark (Kupka et al., 2016). It has been estimated that as yet worldwide more than 5 million children have been born after the use of ART (ESHRE, 2014).

During an in vitro fertilization (IVF) treatment, women are routinely stimulated with hormones for the development of multiple follicles in the ovary. Oocytes are retrieved from the woman and are prepared for fertilization. Also the semen containing the spermatozoa is prepared and fertilization takes place either by conventional IVF (i.e. co-incubation of the oocyte and spermatozoa in a culture dish) or by intracytoplasmic sperm injection (ICSI) (i.e. injection of a single spermatozoon directly into the oocyte). The resulting zygotes are cultured for two to six days after fertilization in embryo culture medium, before embryos of good quality are selected for transfer or cryopreservation.

\section{Embryo culture media}

In the early days of IVF, culture of mammalian preimplantation embryos was performed in tissue culture media that were adapted for embryo culture, such as Whitten's medium (Whitten, 1956), BMOC-2 (Brinster, 1963), M16 medium (Whittingham, 1971) and CZB medium (Chatot et al., 1989). Formulations of these media were based on simple salt solutions containing sodium chloride $(\mathrm{NaCl})$, potassium chloride $(\mathrm{KCl})$, calcium chloride $\left(\mathrm{CaCl}_{2}\right)$, sodium bicarbonate $\left(\mathrm{NaHCO}_{3}\right)$, sodium phosphate $\left(\mathrm{NaH}_{2} \mathrm{PO}_{4}\right)$ or potassium phosphate $\left(\mathrm{KH}_{2} \mathrm{PO}_{4}\right)$, magnesium chloride $\left(\mathrm{MgCl}_{2}\right)$ or magnesium sulfate $\left(\mathrm{MgSO}_{4}\right)$, with the addition of glucose [Whitten's and M16], lactate and pyruvate [BMOC-2, M16 and CZB], ethylenediaminetetraacetic acid (EDTA) and glutamine [CZB], and bovine serum albumin (BSA) [Whitten's, BMOC-2, M16 and CZB] (Baltz, 2012; Chronopoulou and Harper, 2015). The subsequent development of the embryo culture media followed either the 'back to nature' principle or the 'let the embryo choose' principle. In accordance with the 'back to nature' principle, the formulations of 
media were based on the chemical compositions of oviduct and uterine fluids, which resulted in the formulation of Human Tubal Fluid (HTF) medium (Quinn et al., 1985). The move to extended culture and transfer at the blastocyst stage (day 5 or 6 after fertilization), and the changes in the metabolic activity of embryos during this period resulted in the formulation of sequential culture media (Gardner, 1998). These sequential culture media systems include distinct formulations for fertilization (day 0), cleavage stages (day 0 to day 3 after fertilization) and blastocyst stages (day 3 to day 5 or 6 after fertilization) (Quinn, 2003). In accordance with the 'let the embryo choose' principle, a computer model using simplex optimization was applied to determine the optimal concentration of each component by measuring the response (i.e. the proportion of mouse embryos that developed beyond the two-cell stage) to simultaneously varying the concentrations of components in the initial medium (Lawitts and Biggers, 1991). Twenty cycles of optimization resulted in the formulation of Simplex Optimization Medium (SOM), which was later on slightly modified and called KSOM (Erbach et al., 1994) and finally modified into KSOMaa after the addition of amino acids (Summers et al., 2000). Nowadays, most commercially available culture media for human IVF are based on one of the two principles and consist of a mixture including salts, energy sources (e.g. glucose, lactate and pyruvate), proteins (e.g. albumin), antibiotics, amino acids and vitamins.

\section{Developmental origin of health and disease}

According to the Barker or "Developmental Origin of Health and Disease" (DOHaD) hypothesis, the fetus in utero is sensitive to its environment and adaptations of the fetus to its environment have lasting consequences for its development, growth and future health. It has been found that there is an inverse association between birth weight and coronary heart disease and the related disorders of stroke, hypertension and type 2 diabetes in adult life (Barker, 1997). In the Dutch famine study it has been observed that individuals who had been exposed to famine as a fetus in early gestation had higher rates of coronary heart disease, a more atherogenic lipid profile, disturbed blood coagulation, increased stress responsiveness and that they were more often obese in adult life than individuals who had been exposed to famine as a fetus in mid- or late-gestation, and non-exposed individuals (Roseboom et al., 2006). It has also been suggested that, already during the periconceptional period, gametes and preimplantation embryos adapt to their environment with long-term health consequences (Kwong et al., 2000). A classic example are the studies in which a maternal low-protein diet is given to pregnant mice or rats during the preimplantation period after mating and a normal diet for the remainder of pregnancy and during weaning. This resulted in offspring with increased weight, sustained hypertension, and 
abnormal anxiety-related behavior (Kwong et al., 2000; Watkins et al., 2008; Watkins et al., 2010; Watkins et al., 2011). In sheep, maternal under-nutrition around the periconceptional and preimplantation periods affects fetal and placental growth (MacLaughlin et al., 2005). The sensitivity of gametes and preimplantation embryos to their environment imposes potential risks in relation to embryo culture media in IVF.

\section{Health outcome in IVF children}

It is well known that children born after the application of assisted reproductive technologies (ART) have an increased risk of several adverse perinatal outcomes compared with naturally conceived children. Part of this increased risk was associated with the increased multiple pregnancy rate after ART due to the transfer of more than one embryo (van Montfoort et al., 2006). Therefore, elective single embryo transfer (eSET) was introduced to reduce the number of multiple pregnancies (Vilska et al., 1999). However, also in IVF singletons an increased risk has been found for perinatal mortality, preterm birth, small for gestational age, low birth weight, and birth defects when compared with naturally conceived singletons with relative risks ranging between 1.39 and 1.87 (Helmerhorst et al., 2004; Jackson et al., 2004; Rimm et al., 2004; McDonald et al., 2009; Pandey et al., 2012; Pinborg et al., 2013; Fauser et al., 2014). A limited amount of data exists regarding the postnatal health and development in IVF children. In a systematic review it was concluded that there is reassuring evidence concerning the health outcome in IVF children, as it is very similar to that of naturally conceived children regarding childhood cancer risk, respiratory and allergic disorders, ophthalmological and auditory disorders, growth and pubertal development, and various mental health outcomes (Hart and Norman, 2013a; 2013b). However, there are some indications that IVF children have a poorer cardiovascular and metabolic outcome than naturally conceived children, including elevated systolic and diastolic blood pressures, higher fasting glucose levels, vascular dysfunction and increased central adiposity (Ceelen et al., 2008; Sakka et al., 2010; Belva et al., 2012; Scherrer et al., 2012; Valenzuela-Alcaraz et al., 2013; Seggers et al., 2014; Pontesilli et al., 2015).

\section{Pre- and postnatal effects of embryo culture media}

The adverse outcome in IVF children may be related to patient-related factors such as the age of the mother and the infertility of the parents, and to specific aspects of the ART technique such as the hormonal stimulation and culture conditions (Henningsen and Pinborg, 2014). The medium that is used for oocyte and embryo culture in IVF/ICSI treatments is considered to be an important 
factor, as it is the direct environment of the growing embryo. From animal studies, it is clear that culture medium constituents can have profound effects on the perinatal outcome of the offspring. For example, serum supplementation can lead to an increase in fetal weight in cattle and sheep (Thompson et al., 1995; Young et al., 1998; Rooke et al., 2007) and reduced fetal weight in mice (Khosla et al., 2001a). More studies using mouse models found a change in fetal and birth weight after in vitro culture in different media when compared with in vivo controls (Bowman and McLaren, 1970; Sjoblom et al., 2005; Delle Piane et al., 2010; Scott et al., 2010; Le et al., 2013). Also postnatal effects have been observed including behavioral defects (Ecker et al., 2004; Fernandez-Gonzalez et al., 2004), glucose intolerance and insulin resistance (Scott et al., 2010; Donjacour et al., 2014), abnormal fat and liver metabolism and hypertension (Watkins et al., 2007) in the offspring.

Earlier studies from our group indicated that culture media in human IVF could affect birth weight of the children (Dumoulin et al., 2010; Nelissen et al., 2012), which has been both supported and disputed since then in studies comparing other culture media (Zandstra et al., 2015). However, these studies were observational and were not very robust due to weaknesses in study design, such as a non-randomized design, retrospective design, use of different culture media over consecutive periods of time, historical controls and/or relatively small sample sizes. In contrast to the clear effects of culture medium constituents on the perinatal outcome of the offspring in animal models, it is still unclear to what extent culture media have an effect on the offspring in humans. Even less is known on whether culture medium has an effect on the postnatal development in humans.

It is important that culture media provide a proper environment to support the first few days of in vitro embryo development, as these initial development stages might have long-term effects. It has been demonstrated that culture media can deteriorate during storage (Stewart-Savage and Bavister, 1988; Weathersbee et al., 1995; Hossain et al., 2010). Wales and Whittingham (1971) found a decreased blastocyst formation in mice when embryos were cultured in medium that had been stored at $5^{\circ} \mathrm{C}$ for 1 month, however, others showed that culture in Ham's F10 and M16 media that had been stored at $4^{\circ} \mathrm{C}$ for several months (up to 14 months) resulted in no decreased potential of mouse embryos to develop to the blastocyst stage (Naz et al., 1986; Shioda and Tsunoda, 1986; De Silva, 1993). Little is known about the decreasing ability of culture media to maintain proper embryo development in humans. One example of deterioration is the spontaneous break down of amino acids, especially free L-glutamine, into ammonium (as one of the substances produced) during incubation at $37^{\circ} \mathrm{C}$ (Gardner and Lane, 1993). Ammonium has been found to be detrimental for embryo development and to 
result in reduced fetal development potential and pregnancy rates in animal models (Gardner and Lane, 1993; Lane and Gardner, 1994; Hammon et al., 2000; Lane and Gardner, 2003; Sinawat et al., 2003; Zander et al., 2006; Tareq et al., 2007; Yuan and Krisher, 2010; Gardner et al., 2013) and in humans (Virant-Klun et al., 2006; Hashimoto et al., 2008; Gardner et al., 2013; Li et al., 2013). Although the free L-glutamine has been replaced by a more stable form of glutamine, i.e. a dipeptide form such as alanyl-glutamine or glycyl-glutamine, in most presently used embryo culture media, the formation of ammonium has not been completely eliminated in these media.

\section{Epigenetic regulation}

During the earliest stages of embryonic development, at the time that IVF procedures like embryo culture are applied, critical epigenetic reprogramming takes place. Epigenetic (re)programming refers to heritable changes in gene expression by mechanisms other than changes in the underlying DNA sequence, which determines the phenotype. Epigenetic reprogramming involves the erasure and remodeling of epigenetic marks, such as DNA methylation and histone modifications, during mammalian development in order to form totipotent cells and downregulate the inheritance of epigenetic information between generations (Reik et al., 2001). Epigenetic marks can respond to intrinsic and environmental signals and adapt accordingly throughout life. The culture medium is the direct environment of the growing embryo and might affect the epigenetic regulation resulting in altered gene expression profiles. Several studies with animal models indeed indicate that this is the case. Gene expression has been found to be affected by the type of culture medium or supplementation used (Khosla et al., 2001a; Fernandez-Gonzalez et al., 2004; Rinaudo and Schultz, 2004; Saadeldin et al., 2011; Cagnone et al., 2012; Schwarzer et al., 2012; Arias et al., 2013) and it has been found that culture medium components can interfere with the epigenetic regulation of genes by changing the DNA methylation (Doherty et al., 2000; Khosla et al., 2001a; Mann et al., 2004; Market-Velker et al., 2010). However, studies on the effect of embryo culture media on the human embryonic transcriptome are lacking.

\section{Outline of the thesis}

The goal of this thesis is to assess if embryo culture media affect perinatal and postnatal outcome, which components of the culture media might play an important role in this, and what the mode of action may be. 
The first aim of the thesis is to investigate whether human embryo culture media used in IVF during the first few days of preimplantation development affect perinatal and postnatal outcome. Therefore, in Chapter $\mathbf{2}$ we study the effect of two commonly used culture media ( $G 5$ and HTF) on success rate and perinatal outcome in a multicenter randomized controlled trial. The effect on postnatal outcome is evaluated in Chapter $\mathbf{3}$ as we investigate if postnatal growth during the first 2 years of life in IVF singletons is affected by the type of medium (Cook or G3) used for culturing human embryos.

To investigate our hypothesis that culture media might lose their ability to properly support embryo development by deterioration of components, we investigate the effect of age of G5 embryo culture medium on embryo development, ongoing pregnancy and birth weight of the newborns (Chapter 4). Furthermore, in Chapter $\mathbf{5}$ we assess the ammonium accumulation in 26 commercially available embryo culture media and 8 protein supplements during storage at $2-8^{\circ} \mathrm{C}$ and during incubation at $37^{\circ} \mathrm{C}$.

Finally, we explore if the medium used for embryo culture in vitro during an IVF treatment affect the epigenetic regulation. Alongside the randomized controlled trial from Chapter 2, we carry out a microarray analysis on the gene expression of human blastocysts and identify pathways that show a culture medium-dependent activity (Chapter 6). 



\section{CHAPTER 2}

\section{Influence of embryo culture medium (G5 and HTF) on pregnancy and perinatal outcome after in vitro fertilization: a multicenter randomized controlled trial}

Sander H.M. Kleijkers*, Eleni Mantikou*, Els Slappendel, Dimitri Consten, Jannie van Echten-Arends, Alex M. Wetzels, Madelon van Wely, Luc J.M. Smits, Aafke P.A. van Montfoort, Sjoerd Repping, John C.M. Dumoulin*, Sebastiaan Mastenbroek*

Human Reproduction 2016, in press

* These authors contributed equally to this article 


\section{Abstract}

Study question: Does embryo culture medium influence pregnancy and perinatal outcome in IVF?

Summary answer: Embryo culture media used in IVF not only affect treatment efficacy but also the birth weight of newborns.

What is known already: A wide variety of culture media for human preimplantation embryos in IVF/ICSI treatments currently exists. It is unknown which medium is best in terms of clinical outcomes. Furthermore, it has been suggested that the culture medium used for the in vitro culture of embryos affects birth weight, but this has never been demonstrated by large randomized trials.

Study design, size, duration: We conducted a multicenter, double-blind randomized controlled trial comparing the use of HTF and G5 embryo culture media in IVF. Between July 2010 and May 2012, 836 couples (419 in the HTF group and 417 in the $\mathrm{G} 5$ group) were included. The allocated medium was used in all treatment cycles a couple received within one year after randomization, including possible transfers with frozen-thawed embryos.

Participants/materials, setting, methods: Couples that were scheduled for an IVF or ICSI treatment at one of the six participating centers in the Netherlands or their affiliated clinics.

Main results and the role of chance: The live birth rate was higher, albeit nonsignificantly, in couples assigned to G5 than in couples assigned to HTF $(44.1 \%$ (184/417) vs. $37.9 \%$ (159/419); RR: 1.2; $95 \% \mathrm{Cl}: 0.99$ to 1.37). Number of utilizable embryos, implantation rate and clinical pregnancy rate were significantly higher for couples assigned to G5. Of the 383 live born children in this trial, birth weight data from 380 children (300 singletons (G5: 163, HTF: 137) and 80 twin children (G5: 38, HTF: 42)) were retrieved. Birth weight was significantly lower in the G5 group compared with the HTF group, with a mean difference of $158 \mathrm{~g}(95 \% \mathrm{Cl}: 42-$ $275 \mathrm{~g} ; \mathrm{P}=0.008)$. More singletons were born preterm in the G5 group (8.6\% $(14 / 163)$ vs. $2.2 \%(3 / 137)$, but singleton birth weight adjusted for gestational age and gender ( $\mathrm{z}$-score) was also lower in the G5 than HTF group $(-0.13 \pm 0.08$ vs. $0.17 \pm 0.08 ; P=0.008)$.

Limitations, reasons for caution: This study was powered to detect a $10 \%$ difference in live births while a smaller difference could still be clinically relevant. The effect of other culture media on perinatal outcome remains to be determined. 
Wider implications of the findings: Embryo culture media used in IVF not only affect treatment efficacy but also perinatal outcome. This suggests that the millions of human embryos that are cultured in vitro each year are highly sensitive to their environment. These findings should lead to increased awareness, mechanistic studies and legislative adaptations to protect IVF offspring during the first few days of their existence.

Study funding/competing interest(s): This project was partly funded by The NutsOhra foundation (Grant 1203-061) and March of Dimes (Grant 6-FY13-153). The authors declare no conflict of interest.

Trial registration number: NTR1979 (Netherlands Trial Registry).

Trial registration date: September $1^{\text {st }} 2009$.

Date of first patient's enrolment: July $18^{\text {th }} 2010$. 


\section{Introduction}

Subfertility is of major clinical and social concern. The most frequently used treatments of subfertility are in vitro fertilization (IVF) and intracytoplasmic sperm injection (ICSI). The American and European registries of assisted reproductive technology reported a total of 697,556 initiated assisted reproductive technology (ART) cycles in the year 2010 (Centers for Disease Control and Prevention, 2012; Kupka et al., 2014). Despite their common use, IVF and ICSI are far from optimal treatments; in the USA and Europe a delivery rate per started cycle was reported of only $29 \%$ and $24 \%$, respectively (Centers for Disease Control and Prevention, 2012; Kupka et al., 2014).

The medium that is used for embryo culture in IVF/ICSI treatments is considered to play an important role in IVF/ICSI success rates and treatment outcomes. The choice of embryo culture medium has been found to affect embryo quality and pregnancy chances (Quinn et al., 1985; Utsunomiya et al., 2002; Balaban and Urman, 2005; Hoogendijk et al., 2007; Cossiello et al., 2012; Mantikou et al., 2013b). However, a recent systematic review showed that randomized studies that compare clinical outcomes of different culture media are limited in number and of low methodological quality (Mantikou et al., 2013b; Youssef et al., 2015). Thus, it is yet unknown which culture medium leads to the highest live birth rates after IVF/ICSI, the outcome generally considered to be most relevant for evaluating IVF programs (Mantikou et al., 2013b).

Children born after assisted reproductive technology (ART) are at increased risk of adverse perinatal outcomes such as preterm birth (PTB) and low birth weight (LBW) when compared with naturally conceived children (Pandey et al., 2012; Fauser et al., 2014; Henningsen and Pinborg, 2014). This may be due to both intrinsic patient factors and specific aspects of the IVF technique (Pinborg et al., 2013).

Initial reports from a semi-randomized data-analysis suggested that culture media in IVF could affect birth weight of the children (Dumoulin et al., 2010; Nelissen et al., 2012). The difference in growth was already detectable during the second trimester of pregnancy (Nelissen et al., 2013) and was present at least up to the age of two years (Kleijkers et al., 2014). These findings have, since then, been both supported and disputed in studies comparing other culture media (Zandstra et al., 2015).

We conducted a large multicenter, randomized, double-blind trial comparing live birth rates as well as pregnancy and perinatal outcomes after IVF using one of two 
culture media that have been used worldwide in IVF programs, HTF medium and G5 medium.

\section{Materials and methods}

\section{Trial design}

In this multicenter randomized controlled trial, couples were randomly assigned to have their oocytes and embryos cultured in one of the two media, i.e. G5 (Vitrolife, Göteborg, Sweden) or HTF (Lonza, Verviers, Belgium). The end of study participation for a couple was the achievement of a live birth, the passing of one year after randomization, or withdrawal of consent by the couple. The study was conducted at six hospital-based IVF centers in the Netherlands (Academic Medical Center in Amsterdam (AMC), Catharina Hospital in Eindhoven, St. Elisabeth Hospital in Tilburg, Maastricht University Medical Center in Maastricht (MUMC), University Medical Center Groningen in Groningen (UMCG), and Radboud university medical center in Nijmegen (UMCN)) and four of their affiliated clinics (Onze Lieve Vrouwe Gasthuis in Amsterdam, Gemini Hospital in Den Helder, Scheper Hospital in Emmen, and Maxima Medical Center in Veldhoven). The study protocol was approved by the Central Committee on Research Involving Human Subjects (CCMO) in the Netherlands and by the institutional review boards of all participating centers. The study was registered in the Netherlands Trial Registry (NTR1979).

\section{Randomization}

Randomization was performed centrally by an online computer program with a 1:1 allocation using random block sizes of two and four couples, one day before oocyte retrieval of the first cycle. For each individual center, stratification was performed for fertilization technique (IVF or ICSI) and maternal age ( $<38$ and $\geq 38$ years of age). Allocation sequence and allocated treatment were fully blinded to participating couples, attending gynecologists, fertility doctors and outcome assessors. Blinding of the embryologists was not possible since they performed the procedures in the laboratory. At the end of the study, when data collection was completed, the allocation sequence was revealed to the primary investigators.

\section{Participants}

Couples that were scheduled for an IVF or ICSI treatment for their first IVF/ICSI cycle ever or first IVF/ICSI cycle after a previous successful pregnancy, were 
eligible to participate in the study. Couples undergoing a modified natural cycle, couples for whom IVF was used to prevent the transmission of HIV, couples undergoing preimplantation genetic diagnosis (PGD) and couples using assisted reproductive technology for fertility preservation were excluded. All participating couples provided written informed consent for participating in the study and the use of their data.

\section{Interventions and IVF procedures}

Participating couples were randomly assigned to either the G5 culture medium group or HTF culture medium group. The allocated medium was used in all treatment cycles, including transfers with cryopreserved embryos that the woman received in the year following randomization. All other treatment procedures, such as ovarian hyperstimulation, follicular aspiration and oocyte fertilization, were performed according to the routine IVF/ICSI procedures in each particular center and were identical for both study groups.

The G5 PLUS culture medium system included G-IVF PLUS medium for fertilization, G1 PLUS medium for culturing embryos from day one to day three and G2 PLUS medium for culturing embryos from day three to day four where applicable. The G5 PLUS media were ready-to-use media already supplemented with human serum albumin (HSA). HTF medium was used for culture of oocytes and embryos from fertilization up to day four of culture where applicable. In order to keep procedures as similar as possible HTF embryos were transferred to a new culture dish containing fresh HTF on the third day of culture. HTF was supplemented with $10 \%$ pasteurized plasma protein solution (Central Laboratory of Blood Transfusion, Amsterdam, the Netherlands).

In case of IVF, oocytes were incubated in dishes containing G-IVF PLUS or HTF, according to the culture medium allocated to the couple, with 10,000 - 100,000 progressively motile spermatozoa for fertilization. The next morning, cumulus cells were removed and all oocytes were transferred to a clean dish containing the allocated medium, G1 PLUS or HTF. On day three of culture the embryos were transferred to a new dish containing G2 PLUS or HTF.

In case of ICSI, the oocytes were denudated using cumulase (Origio, Malov, Denmark), hyadase (Origio, Malov, Denmark), or hyase (Vitrolife, Göteborg, Sweden), injected with a single immobilized spermatozoon and directly thereafter cultured in dishes containing G1 PLUS or HTF according to treatment allocation. On day three of culture the embryos were transferred to a new dish containing $\mathrm{G} 2$ PLUS or HTF. 
Embryo culture was performed following the manufacturer's instructions, i.e. at $37^{\circ} \mathrm{C}$ and $6 \% \mathrm{CO}_{2}$ for $\mathrm{G} 5$ and $37^{\circ} \mathrm{C}$ and $5 \% \mathrm{CO}_{2}$ for HTF. Three centers cultured oocytes and embryos in 5\% $\mathrm{O}_{2}$ (AMC, MUMC and St. Elisabeth Hospital), while the other three cultured oocytes and embryos in air (18-20\% $\mathrm{O}_{2}$; Catharina Hospital, UMCG and UMCN). Embryo morphology was assessed daily and the number of cells as well as the percentage of fragmentation were scored based on a structured scoring sheet available in all centers (Alpha Scientists in Reproductive Medicine and ESHRE Special Interest Group of Embryology, 2011). All embryologists in all centers participate in a national external online embryo scoring quality control scheme to limit inter-individual variation in embryo scoring (www.embryo-online.eu). Embryos of sufficient quality were transferred to the uterine cavity of the women on day 2 (UMCG, MUMC) or 3 (AMC, Catharina Hospital, MUMC, St. Elisabeth Hospital, UMCN) after fertilization according to the local policies of each participating center. Cryopreservation of supernumerary good quality embryos was performed according to local protocol on day 3 (MUMC, UMCG, UMCN), 4 (AMC, Catharina Hospital, St. Elisabeth Hospital) or 5 (St. Elisabeth Hospital) after fertilization. After thawing, the allocated medium was used for culture and transfer of these embryos. A new IVF/ICSI cycle was initiated only after all cryopreserved embryos from the previous cycle were thawed and transferred.

\section{Outcomes}

The primary outcome was live birth rate, which was defined as the proportion of couples that achieved a birth of at least one baby born alive, independent of gestational age. Secondary outcomes were number of utilizable embryos, implantation rate, biochemical pregnancy, clinical pregnancy, ongoing pregnancy, miscarriage, birth weight and congenital abnormalities. The number of utilizable embryos was defined as the number of embryos transferred plus the number of embryos cryopreserved. The number of implantations was determined by the number of fetal sacs as identified by transvaginal ultrasound examination at 6-8 weeks of gestation. A biochemical pregnancy was defined as a serum $\beta$ human chorionic gonadotropin level of at least $50 \mathrm{IU}$ per liter two weeks after embryo transfer. Clinical pregnancy was determined by the presence of a gestational sac and fetal heartbeat confirmed by transvaginal ultrasound examination at 6-8 weeks of gestation. Ongoing pregnancy was defined as a viable intrauterine pregnancy after 12 weeks of gestation. A miscarriage was determined by a biochemical pregnancy not resulting in a live birth.

After delivery, questionnaires were sent to all women and their obstetrician or midwife. Questionnaires included questions about ultrasound examinations, pregnancy characteristics and complications (i.e. gestational diabetes, 
hypertension and pre-eclampsia) and perinatal outcome. Congenital malformations were classified as major when they caused functional impairment or required surgical correction. The remaining congenital malformations were considered minor (Bonduelle et al., 2002). Actual gestational age (GA) was calculated based on the day of oocyte retrieval, which was defined as Day 14 of the cycle. Birth weight percentiles were based on the median weight of a reference population of children born at the same GA and of the same gender (Oken et al., 2003). These were used to define very small for GA (<3rd percentile), small for GA (<10th percentile), large for GA ( $>90$ th percentile) and very large for GA ( $>97$ th percentile) infants and to calculate z-scores. In case of a twin pregnancy, the inter-twin birth weight disparity was calculated in grams and the inter-twin birth weight discordance as a percentage by dividing the difference in birth weight by the weight of the heaviest twin and multiplying the result by $100 \%$ (Blickstein and Kalish, 2003).

To investigate fetal growth, data from the ultrasound pregnancy examinations were used. In the Netherlands, all pregnant women are offered ultrasound dating during the first trimester and are counseled for fetal ultrasound examination around 20 weeks of gestation to have fetal biometry performed and be screened for structural abnormalities. Furthermore, women can freely choose to participate in first-trimester screening for Down syndrome. To compare fetal growth between the study groups, while adjusting for the differences in GA at which the examinations were performed, differences between the estimated GA (based on data from the ultrasound examinations) and actual GA (oocyte retrieval based) were used $(\triangle G A)$ and expressed in days. The crown-rump-length (CRL)-based formula by Hadlock et al. (1992) was used to calculate the GA at 8 and 12 weeks of gestation and the biparietal diameter (BPD)-based formulae by Mul et al. (1996) \#1 and \#2 and Selbing and Kjessler (1985) were used to calculate the GA at 20 weeks of gestation (Saltvedt et al., 2004). Furthermore, fetal weight was estimated based on several sonographic parameters (head circumference $(H C)$, abdominal circumference ( $A C)$, femur length ( $F L$ ) and $B P D$ ) that were measured during the mid-trimester ultrasound scan at 20 weeks of gestation by using the Hadlock et al. (1985) I, III and IV formulae (Hoopmann et al., 2010).

\section{Sample size and interim analysis}

Based on a cumulative live birth rate of $45 \%$ after one year of IVF/ICSI treatment in the participating centers in the years preceding this study, we calculated that at least 784 women would be needed to detect a difference of $10 \%$ in live birth rate after one year of treatment with a power of $80 \%$ at an alpha of 0.05 . More patients were counseled and invited to participate in the study to allow for dropouts. 
One interim analysis of efficacy was planned to be performed one year after the initiation of the study by an independent data and safety monitoring committee. Primary outcome of this interim analysis was ongoing pregnancy for first, second, and third cycles, including transfers with frozen-thawed embryos, that had been performed at that time. The data and safety monitoring committee had to use these proxy outcomes as otherwise an interim analysis would not have been possible before the completion of the inclusion of the study. Live birth data were only available at the end of the study, due to the design and inclusion rate of the study. For the same reason no formal stopping rules or adjusted statistics were applied. A blinded overview, which was available only to the data and safety monitoring committee, was used for the interim analysis.

\section{Statistical analysis}

We calculated rates of pregnancy and live birth in each group and the corresponding rate ratios (RR) with $95 \%$ confidence intervals $(95 \% \mathrm{Cl})$. Differences in outcomes were statistically evaluated using chi-square statistics for categorical variables and one-way ANOVA for continuous variables. Data were analyzed according to the intention-to-treat principle. Birth weight differences between children from the G5 group versus the HTF group were analyzed by means of general estimating equations (GEE) (Zeger and Liang, 1986), in view of statistical dependence between birth weights within twin pairs. In addition, being part of a twin was added to the analysis as a co-variable because of its strong association with birth weight. Perinatal outcome was further investigated in singletons and twins separately, as beforehand twinning was considered to be an important confounder and a treatment related bias as the study was not designed to include single embryo transfers only. To investigate the effect of medium on birth weight controlled for other potential confounders, a multivariable regression analysis was performed. Imputation was used for missing data on parental height, weight and smoking.

\section{Results}

Between July 2010 and May 2012, a total of 836 couples were randomly allocated to undergo IVF/ICSI using either G5 $(n=417)$ or HTF $(n=419)$ medium for embryo culture (Figure 1). Four-hundred-and-twelve couples in the G5 group and 395 couples in the HTF group underwent the allocated intervention in all their cycles. Five couples in the G5 and twenty-four couples in the HTF group did not receive the allocated treatment in one of the performed cycles because of human error in identifying the couple as participating in the study; in these couples the medium used for non-trial participants in that specific center was used. Only one couple (in 
the $\mathrm{G} 5$ group) withdrew consent prior to finishing the year of treatment, after two cycles. The couple had not achieved pregnancy and was analyzed as such. Nineteen couples were allocated in either of the two culture media without fulfilling the inclusion criteria; the primary reason being that they had undergone one or more previous unsuccessful IVF/ICSI treatments. Since our analysis is based on intention-to-treat, those couples were included in the analysis.

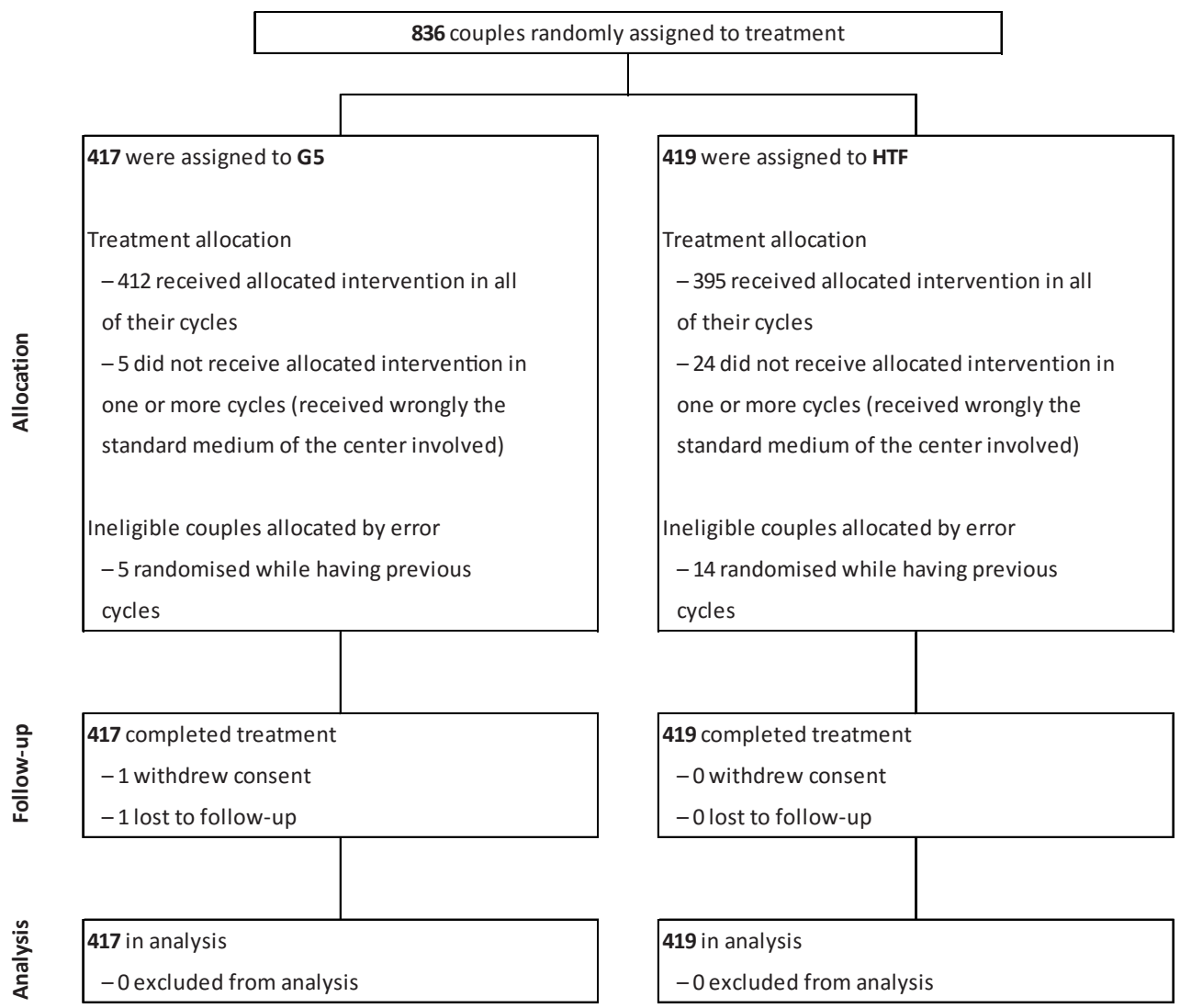

Figure 1. Flow chart of allocation, follow-up and analysis of the included couples.

The couples remained enrolled in the study for one year after allocation and in case of an ongoing pregnancy they were followed until delivery. Only one of the couples with an ongoing pregnancy (in the G5 group) was lost to follow-up. We included this pregnancy as not resulting in a live birth in our analysis. The baseline characteristics of the couples are presented in Table 1. Four couples (three in the G5 group and one in the HTF group) received oocyte donation and twenty-two couples (eight in the G5 group and fourteen in the HTF group) had treatment with sperm from a donor. In these cases, the age of the donor was used for the calculation of the mean maternal and paternal age. No important differences 
were found between baseline characteristics of the G5 group and the HTF group, either among all couples or in the subgroup of couples with a live born child (Table 1).

Table 1. Characteristics of the couples.

\begin{tabular}{|c|c|c|c|c|}
\hline \multirow[t]{2}{*}{ Characteristic } & \multicolumn{2}{|l|}{ All couples } & \multicolumn{2}{|c|}{ Couples with a live born child } \\
\hline & G5 $(n=417)$ & $\operatorname{HTF}(n=419)$ & G5 $(n=184)$ & $\operatorname{HTF}(n=159)$ \\
\hline \multicolumn{5}{|l|}{ Primary indication for IVF/ICSI } \\
\hline Tubal & $47(11.3)$ & $37(8.8)$ & 20 (10.9) & $12(7.5)$ \\
\hline Male subfertility & $211(50.6)$ & $225(53.7)$ & $104(56.5)$ & $94(59.1)$ \\
\hline Unexplained & $100(24.0)$ & $84(20.0)$ & $34(18.5)$ & $28(17.6)$ \\
\hline Other & $59(14.1)$ & $73(17.4)$ & $26(14.1)$ & $25(15.7)$ \\
\hline \multicolumn{5}{|l|}{ Maternal characteristics } \\
\hline Age (years) & $33.9 \pm 4.3$ & $33.8 \pm 4.4$ & $32.9 \pm 3.9$ & $33.0 \pm 4.1$ \\
\hline Height $(\mathrm{cm})^{*}$ & $\mathrm{n} / \mathrm{a}$ & $\mathrm{n} / \mathrm{a}$ & $169.8 \pm 7.6$ & $171.0 \pm 7.0$ \\
\hline Weight $(\mathrm{kg})^{*}$ & $\mathrm{n} / \mathrm{a}$ & $\mathrm{n} / \mathrm{a}$ & $68.6 \pm 12.6$ & $71.1 \pm 12.7$ \\
\hline Smoking $\geq 10$ cigarettes/day* & $\mathrm{n} / \mathrm{a}$ & $\mathrm{n} / \mathrm{a}$ & $13(7.1)$ & $16(10.1)$ \\
\hline \multicolumn{5}{|l|}{ Paternal characteristics } \\
\hline Age (years) & $37.5 \pm 6.2$ & $37.3 \pm 6.4$ & $36.7 \pm 6.2$ & $37.0 \pm 6.4$ \\
\hline Height $(\mathrm{cm})^{*}$ & $\mathrm{n} / \mathrm{a}$ & $\mathrm{n} / \mathrm{a}$ & $183.1 \pm 7.5$ & $182.9 \pm 7.6$ \\
\hline Weight $(\mathrm{kg})^{*}$ & $\mathrm{n} / \mathrm{a}$ & $\mathrm{n} / \mathrm{a}$ & $87.7 \pm 13.6$ & $88.1 \pm 13.3$ \\
\hline Smoking $\geq 10$ cigarettes/day* & $\mathrm{n} / \mathrm{a}$ & $\mathrm{n} / \mathrm{a}$ & $26(14.1)$ & $21(13.2)$ \\
\hline Duration of subfertility (years) & $3.1 \pm 1.9$ & $3.1 \pm 2.3$ & $3.1 \pm 1.8$ & $3.3 \pm 2.5$ \\
\hline Nulliparous & $312(74.8)$ & $317(75.7)$ & $133(72.3)$ & $115(72.3)$ \\
\hline
\end{tabular}

Data presented as mean \pm SD or number (\%). N/A denotes not available.

* Data on maternal height, maternal weight, maternal smoking, paternal height, paternal weight and paternal smoking were missing from 26 (7.6\%), 28 (8.2\%), 28 (8.2), $27(7.9 \%), 32$ (9.3\%) and 38 (11.1\%) couples, respectively.

\section{IVF outcomes}

After one year of treatment, live birth rate was not significantly different for women in the G5 group as compared with women in the HTF group $144.1 \%$ (184/417) vs. $37.9 \%$ (159/419); RR: 1.2; 95\% Cl: 0.99 to 1.37; P = 0.08). Similarly, there was no difference in the percentage of women achieving an ongoing pregnancy between the G5 group and the HTF group (45.3\% (189 of 417) vs. $38.7 \%$ (162 of 419); RR: $1.2 ; 95 \% \mathrm{Cl}: 0.99$ to $1.37 ; \mathrm{P}=0.06)$. Women in the G5 group had a significantly higher rate of clinical pregnancy than women in the HTF group (47.7\% (199 of 417) vs. $40.1 \%$ (168 of 419); RR: $1.2 ; 95 \% \mathrm{Cl}: 1.02$ to 1.39; P = $0.03)$, while the proportion of miscarriages did not differ between the study groups (Table 2 ). 
Chapter 2

Table 2. Outcomes in women allocated to G5 and HTF.

\begin{tabular}{|c|c|c|c|c|}
\hline Outcome & $\mathrm{G} 5(\mathrm{n}=417)$ & $\operatorname{HTF}(n=419)$ & Risk Ratio $(95 \% \mathrm{Cl})^{*}$ & P-value \\
\hline Women with $\geq 1$ child born (live birth rate) & $184(44.1)$ & 159 (37.9) & $1.2(0.99-1.37)$ & 0.08 \\
\hline Total no. of live births ${ }^{£}$ & 203 & 180 & & \\
\hline Women with $\geq 1$ ongoing pregnancy ${ }^{\S}$ & $189(45.3)$ & $162(38.7)$ & $1.2(0.99-1.37)$ & 0.06 \\
\hline Total no. of ongoing pregnancies & 190 & 163 & & \\
\hline Women with $\geq 1$ clinical pregnancy & $199(47.7)$ & $168(40.1)$ & $1.2(1.02-1.39)$ & 0.03 \\
\hline Total no. of clinical pregnancies & 205 & 170 & & \\
\hline Women with $\geq 1$ biochemical pregnancy & $236(56.6)$ & $210(50.1)$ & $1.1(0.99-1.28)$ & 0.06 \\
\hline Total no. of biochemical pregnancies & 255 & 221 & & \\
\hline Women with $\geq 1$ miscarriage & $66(15.8)$ & $56(13.4)$ & $1.2(0.85-1.65)$ & 0.33 \\
\hline Total no. of miscarriages & 71 & 62 & & \\
\hline
\end{tabular}

Data presented as number (\%).

* Risk ratio for G5 group as compared with HTF group. Cl denotes confidence interval.

${ }^{\mathrm{f}} 165$ singleton and 38 twin children in the G5 group; 138 singleton and 42 twin children in the HTF group.

${ }^{\S} 2$ pregnancies with stillborn, 2 pregnancies with intra uterine deaths and 1 pregnancy loss-to-follow-up in the G5 group; 1 pregnancy with stillborn (twin) and 2 pregnancies with intra uterine deaths in the HTF group.

A total of 1497 cycles (713 (mean of 1.71) for the G5 group and 784 (mean of 1.87) for the HTF group) were performed (1.71 \pm 0.04 vs. $1.87 \pm 0.04 ; P=0.003)$. There was no difference in the percentage of cycles with ICSI (58.3\% (416 of 713) vs. 57.3\% (449 of 784); RR: $1.0 ; 95 \% \mathrm{Cl}: 0.93$ to $1.11 ; \mathrm{P}=0.68$ ) or the proportion of transfers with fresh embryos (93.0\% (663 of 713) vs. $93.5 \%$ (733 of 784); RR: 1.0; 95\% Cl: 0.97 to $1.02 ; \mathrm{P}=0.76$ ) between the G5 group and the HTF group (Supplementary Table 1). The percentage of transfers using cryopreserved embryos in the G5 group was higher than in the HTF group (38.7\% (276 of 713 ) vs. $26.0 \%$ (204 of 784); RR: $1.5 ; 95 \%$ Cl: 1.28 to 1.73; P<0.001) (Supplementary Table 1).

The embryo characteristics of all treatments are shown in Table 3. Fertilization rate was significantly lower in the G5 group as compared with the HTF group (62.9\% (3655 of 5807 ) vs. $69.1 \%$ (4341 of 6286 ); $\mathrm{P}<0.001$ ). The mean number of utilizable embryos per cycle, i.e. the mean number of embryos that were transferred or cryopreserved per cycle, was significantly higher in the G5 group as compared with the HTF group $(2.8 \pm 2.3$ vs. $2.3 \pm 1.8$; $\mathrm{P}<0.001)$. Furthermore, the proportion of embryos that implanted after fresh transfer was significantly higher in the G5 group than in the HTF group (20.2\% (195 of 966) vs. $15.3 \%$ (171 of $1117) ; \mathrm{P}<0.001)$. At the end of the study period, significantly more embryos per woman were still cryopreserved in the G5 group as compared with the HTF group (mean $1.28 \pm 0.12$ vs. $0.79 \pm 0.09 ; P=0.001$ ). 
Table 3. Embryologic characteristics of treatment.

\begin{tabular}{|c|c|c|c|}
\hline Characteristic & G5 & HTF & P-value \\
\hline Cumulus-oocyte complexes - no. (mean no. per cycle \pm SD) & $6461(9.1 \pm 5.3)$ & $7102(9.1 \pm 5.4)$ & 0.99 \\
\hline Oocytes inseminated/injected - no. (mean no. per cycle \pm SD) & $5807(8.1 \pm 5.0)$ & $6286(8.0 \pm 5.0)$ & 0.62 \\
\hline Fertilized oocytes (embryos) ${ }^{*}$ - no. (mean no. per cycle \pm SD) & $3655(5.1 \pm 3.9)$ & $4341(5.5 \pm 4.0)$ & 0.04 \\
\hline Fertilization rate - \% per oocytes inseminated/injected & 62.9 & 69.1 & $<0.001$ \\
\hline Utilizable embryost - no. (mean no. per cycle \pm SD) & $1966(2.8 \pm 2.3)$ & $1779(2.3 \pm 1.8)$ & $<0.001$ \\
\hline $\begin{array}{l}\text { Embryos transferred in a fresh cycle }- \text { no. } \\
\text { (mean no. per transfer } \pm S D \text { ) }\end{array}$ & $966(1.5 \pm 0.6)$ & $1117(1.5 \pm 0.6)$ & 0.19 \\
\hline $\begin{array}{l}\text { Implanted embryos from a fresh cycle - no. (\% of embryos } \\
\text { transferred) }\end{array}$ & $195(20.2)$ & $171(15.3)$ & $<0.001$ \\
\hline Embryos cryopreserved - no. (mean no. per cycle \pm SD) & $1000(1.4 \pm 2.4)$ & $662(0.8 \pm 1.8)$ & $<0.001$ \\
\hline $\begin{array}{l}\text { Cryopreserved embryos transferred }- \text { no. (mean no. per } \\
\text { transfer with cryopreserved embryos } \pm \text { SD) }\end{array}$ & $325(1.2 \pm 0.6)$ & $225(1.1 \pm 0.6)$ & 0.20 \\
\hline $\begin{array}{l}\text { Implanted cryopreserved embryos - no. (\% of cryopreserved } \\
\text { embryos transferred) }\end{array}$ & 35 (10.7) & $25(11.1)$ & 0.89 \\
\hline
\end{tabular}

* 2PN zygotes and 1PN/OPN zygotes that cleaved in 48 hours.

+ Number of embryos transferred in a fresh cycle or cryopreserved.

\section{Perinatal outcome}

Of the 343 pregnancies with live births, 301 were single gestation pregnancies (G5: 164, HTF: 137), while 42 were multiple gestation pregnancies (G5: 20, HTF: 22). In two multiple gestation pregnancies with live births (G5: 1, HTF: 1), one of the fetuses was lost between 12 weeks of gestation (ongoing pregnancy) and birth. From this, a total of 383 live born children were included in the study, 165 singleton and $38(18.7 \%)$ twin children in the G5 group and 138 singleton and 42 (23.3\%) twin children in the HTF group.

Of the 383 live born children, birth weight data from 380 children (300 singletons (G5: 163, HTF: 137) and 80 twin children (G5: 38, HTF: 42)) were retrieved. Birth weight was significantly lower in the G5 group compared with the HTF group, with a mean difference of $158 \mathrm{~g}$ ( $95 \% \mathrm{Cl}: 42-275 \mathrm{~g} ; \mathrm{P}=0.008$ ). Separate analysis for singletons and twins are provided in Tables $4 \mathrm{~A}$ and $4 \mathrm{~B}$, respectively. Birth weight of the singletons was also significantly lower in the G5 group compared with the HTF group ( $3299 \pm 46 \mathrm{~g}$ vs. $3480 \pm 44 \mathrm{~g} ; \mathrm{P}=0.005$ ) while there was no difference in birth weight for twins from the G5 or HTF group. Furthermore, singleton birth weight adjusted for gestational age and gender (z-score) was lower in the G5 than HTF group $(-0.13 \pm 0.08$ vs. $0.17 \pm 0.08 ; P=0.008)$. In the $G 5$ group, more singletons were born preterm (8.6\% (14/163) vs. $2.2 \%$ (3/137); RR: $3.92 ; 95 \% \mathrm{Cl}$ : 1.15-13.37; $\mathrm{P}=0.02)$ and there were more singletons with low birth weight $(<2500 \mathrm{~g})(9.8 \%$ (16/163) vs. $2.9 \%$ (4/137); RR: 3.36; $95 \% \mathrm{Cl}: 1.15-9.82 ; \mathrm{P}=0.02)$. 
The percentage of children with major or minor malformations did not differ between the groups (Tables $4 \mathrm{~A}$ and $4 \mathrm{~B}$ ).

Table 4A. Neonatal outcome of live born singletons.

\begin{tabular}{|c|c|c|c|c|}
\hline Outcome & G5 $(n=163)$ & $\operatorname{HTF}(n=137)$ & Risk Ratio $(95 \% \mathrm{Cl}) *$ & P-value \\
\hline Female & $71(43.6)$ & $65(47.4)$ & $0.92(0.72-1.18)$ & 0.56 \\
\hline Birth weight (g) & $3299 \pm 46$ & $3480 \pm 44$ & & 0.005 \\
\hline Z-score & $-0.13 \pm 0.08$ & $0.17 \pm 0.08$ & & 0.008 \\
\hline Gestational age (GA) at birth (weeks) & $39.2 \pm 0.1$ & $39.4 \pm 0.1$ & & 0.19 \\
\hline Preterm birth (<37 weeks) & $14(8.6)$ & $3(2.2)$ & $3.92(1.15-13.37)$ & 0.02 \\
\hline Very preterm birth (<32 weeks) & $1(0.6)$ & $0(0.0)$ & $\mathrm{n} / \mathrm{a}$ & 1.00 \\
\hline Low birth weight (<2500 g) & $16(9.8)$ & $4(2.9)$ & $3.36(1.15-9.82)$ & 0.02 \\
\hline Low birth weight with $G A \geq 37$ weeks & $6(3.7)$ & $3(2.2)$ & $1.68(0.43-6.60)$ & 0.52 \\
\hline Very low birth weight (<1500 g) & $1(0.6)$ & $0(0.0)$ & $\mathrm{n} / \mathrm{a}$ & 1.00 \\
\hline High birth weight (>4500 g) & $1(0.6)$ & $4(2.9)$ & $0.21(0.02-1.86)$ & 0.18 \\
\hline Small for GA (<10th percentile) & $14(8.6)$ & $5(3.6)$ & $2.35(0.87-6.37)$ & 0.10 \\
\hline Very small for GA (<3rd percentile) & $4(2.5)$ & $1(0.7)$ & $3.36(0.38-29.73)$ & 0.38 \\
\hline Large for GA (>90th percentile) & $15(9.2)$ & $16(11.7)$ & $0.79(0.41-1.54)$ & 0.57 \\
\hline Very large for GA (>97th percentile) & $2(1.2)$ & $7(5.1)$ & $0.24(0.05-1.14)$ & 0.09 \\
\hline Major malformations & $4(2.5)$ & $6(4.4)$ & $0.56(0.16-1.95)$ & 0.52 \\
\hline Minor malformations & $6(3.7)$ & $6(4.4)$ & $0.84(0.28-2.55)$ & 0.78 \\
\hline
\end{tabular}

Data presented as mean \pm SEM or number (\%). N/A denotes not applicable.

* Risk ratio for $\mathrm{G} 5$ group as compared with HTF group. Cl denotes confidence interval.

Table 4B. Neonatal outcome of live born twins.

\begin{tabular}{|c|c|c|c|c|}
\hline Outcome & G5 $(n=38)$ & $\operatorname{HTF}(n=42)$ & Risk Ratio $(95 \% \mathrm{Cl})^{*}$ & P-value \\
\hline Female & $23(60.5)$ & $17(40.5)$ & $1.50(0.96-2.34)$ & 0.12 \\
\hline Gestational age (GA) at birth (weeks) & $35.6 \pm 0.4$ & $35.1 \pm 0.4$ & & 0.47 \\
\hline Preterm birth (<37 weeks) & $26(68.4)$ & $30(71.4)$ & $0.96(0.72-1.28)$ & 0.81 \\
\hline Very preterm birth (<32 weeks) & $4(10.5)$ & $8(19.0)$ & $0.55(0.18-1.69)$ & 0.36 \\
\hline Low birth weight (<2500 g) & $21(55.3)$ & $28(66.7)$ & $0.83(0.58-1.19)$ & 0.36 \\
\hline Low birth weight with $G A \geq 37$ weeks & $4(10.5)$ & $4(9.5)$ & $1.11(0.30-4.12)$ & 1.00 \\
\hline Very low birth weight (<1500 g) & $6(15.8)$ & 5 (11.9) & $1.33(0.44-4.00)$ & 0.75 \\
\hline Major malformations & $1(2.6)$ & $2(4.8)$ & $0.55(0.05-5.85)$ & 1.00 \\
\hline Minor malformations & $1(2.6)$ & $0(0.0)$ & $\mathrm{n} / \mathrm{a}$ & 0.48 \\
\hline Birth weight (g) & $2266 \pm 100$ & $2267 \pm 94$ & & 0.99 \\
\hline Birth weight disparity (g) & $426 \pm 64$ & $289 \pm 40$ & & 0.07 \\
\hline Birth weight discordance (\%) & $17.1 \pm 2.4$ & $12.4 \pm 1.7$ & & 0.11 \\
\hline Birth weight discordance ( $\geq 25 \%)$ & $10(26.3)$ & $8(19.0)$ & $1.38(0.61-3.14)$ & 0.59 \\
\hline
\end{tabular}

Data presented as mean \pm SEM or number (\%). N/A denotes not applicable.

* Risk ratio for $\mathrm{G} 5$ group as compared with HTF group. Cl denotes confidence interval. 
To determine how the effect of culture medium on birth weight relates to the effect of other possible birth weight confounders, a multivariate analysis was performed. Multiple linear regression indicated that the adjusted mean birth weight of singletons was 116 grams lower in the G5 group than in the HTF group (95\% Cl: -212 to $-20 ; P=0.02$ ) (Supplementary Table 2). Additionally, in the subgroup of 283 singletons that were born from term pregnancies ( $\geq 37$ weeks), the adjusted mean birth weight of singletons in the G5 group was 100 grams lower than in the HTF group ( $95 \% \mathrm{Cl}:-198$ to $-2 ; \mathrm{P}=0.04)$. It has been shown that the duration of culture can affect birth weight in humans (Makinen et al., 2013; Zhu et al., 2014b; Kaartinen et al., 2015). Furthermore, it has been suggested that oxygen concentration during culture can influence the effect of culture medium, at least on blastocyst formation in the mouse (Morbeck et al., 2014a). As those factors are related to center of treatment in this study, an additional analysis including center of treatment was performed. It was found that embryo culture medium ( $G 5$ versus HTF) was still associated with singleton birth weight ( $\beta=-117$ g; $95 \% \mathrm{Cl}:-213$ to $-20 ; \mathrm{P}=0.02$ ).

\section{Fetal growth}

To investigate the onset of the intrauterine growth difference between the children from the G5 group and HTF group, the fetal growth patterns in single gestation pregnancies were compared between the study groups. Around 8 weeks of gestation, fetal CRL was retrieved in 149 (90.9\%) single gestation pregnancies from the G5 group and 129 (94.2\%) single gestation pregnancies from the HTF group. Not all women participated in the Down screening around 12 weeks of gestation, fetal CRL was retrieved in only 114 single gestation pregnancies, 60 (36.6\%) in the G5 group and 54 (39.4\%) in the HTF group. In the screening for structural abnormalities of the fetus around 20 weeks of gestation, not all biometric parameters used to calculate the $\triangle G A$ or EFW were measured in all women. For $\triangle \mathrm{GA}$ around 20 weeks of gestation, data from 139 (84.8\%) pregnancies from the G5 group and $110(80.3 \%)$ pregnancies from the HTF group were analyzed, while for EFW, data from 138 (84.1\%) G5 pregnancies and 111 (81.0\%) HTF pregnancies were analyzed. Intrauterine growth of singletons from single gestation pregnancies is presented in Table 5 . The differences between the CRL- or BPD-based GA and actual (oocyte retrieval based) GA were not different between the study groups at 8,12 and 20 weeks of gestation. Furthermore, the estimated fetal weight at 20 weeks of gestation, as calculated with three different formulae by Hadlock, did not differ between the two study groups. 
Table 5. Fetal growth in single gestation pregnancies with a live birth.

\begin{tabular}{lccc}
\hline Outcome & G5 & HTF & P-value \\
\hline 8 weeks of gestation (n) & 149 & 129 & 0.762 \\
$\Delta$ GA; Hadlock (1992) & $-0.4 \pm 0.1$ & $-0.5 \pm 0.2$ & 0.244 \\
12 weeks of gestation (n) & 60 & 54 & 0.360 \\
$\Delta$ GA; Hadlock (1992) & $2.3 \pm 0.7$ & 110 & 0.342 \\
20 weeks of gestation (n) & 139 & $0.7 \pm 0.4$ & 0.421 \\
$\Delta$ GA; Mul et al. (\#1) (1996) & $1.2 \pm 0.4$ & $0.3 \pm 0.4$ & 0.199 \\
$\Delta$ GA; Mul et al. (\#2) (1996) & $0.8 \pm 0.4$ & $3.1 \pm 0.5$ & 111 \\
$\Delta$ GA; Selbing and Kjessler (1985) & $3.7 \pm 0.4$ & $343 \pm 5$ & 0.230 \\
20 weeks of gestation (n) & 138 & $349 \pm 5$ & 0.197 \\
EFW; Hadlock I (g) & $335 \pm 4$ & $340 \pm 5$ & \\
EFW; Hadlock III (g) & $341 \pm 4$ & $331 \pm 4$ & \\
EFW; Hadlock IV (g) & & & \\
\hline
\end{tabular}

Data are presented as mean \pm SEM.

\section{Discussion}

In this randomized double-blinded trial we evaluated the effect of two embryo culture media that are routinely used in IVF/ICSI treatments and found a $6 \%$ difference in live births in favor of G5 medium. Despite the absence of a statistical significance in the difference in live birth rate ( $\mathrm{G} 5$ versus HTF; RR 1.2; $95 \% \mathrm{Cl} 0.99$ 276 1.37), we conclude that G5 provides better treatment success than HTF, as other outcomes that are relevant for IVF and correlate to live birth rate, all significantly favored G5. This includes clinical pregnancy, number of utilizable embryos, number of embryos implanted after fresh transfer and the number of cryopreserved embryos that remained available at the end of the study. The beneficial effect of $\mathrm{G} 5$ on all these outcomes but live birth rate is probably due to the study being powered to detect a $10 \%$ difference in live births. Future designs should include a larger number of patients to be able to establish smaller differences, which are still clinically relevant.

Birth weight was significantly lower in children from the G5 group compared with children from the HTF group, with a mean difference of $158 \mathrm{~g}$ (95\% Cl: $42-275 \mathrm{~g}$; P $=0.008$ ). In previous studies with consecutive or alternate use of different culture media, some reported effects of culture medium on birth weight (Dumoulin et al., 2010; Nelissen et al., 2012; Eskild et al., 2013; Hassani et al., 2013; Zhu et al., 2014a; Zhu et al., 2014b), while others found no effect (Eaton et al., 2012; Vergouw et al., 2012; Carrasco et al., 2013; Lin et al., 2013; Ziebe et al., 2013; Lemmen et al., 2014; Wunder et al., 2014) [see review by Zandstra et al. (2015)]. We now, for the first time by means of a large randomized trial, confirm that 
embryo culture medium can have an effect on birth weight in humans. The clinical relevance of a difference in birth weight after IVF, the mechanism through which a few days of embryo culture affects birth weight nine months later, and the optimal composition of culture media for IVF must now be determined.

There were more preterm births, with lower birth weight, among singletons in the G5 group. This does not fully explain however the observed difference in birth weight as also the z-score (birth weight adjusted for gestational age and gender) was significantly lower in the G5 group than the HTF group. It is well known that children born after ART have a higher risk for preterm birth and low birth weight compared with children born after natural conception (Henningsen and Pinborg, 2014). Our study suggests embryo culture medium composition to be one of the causative factors for this.

The fetal growth patterns from both study groups did not differ up to 20 weeks of gestation, which indicates that the onset of the growth differentiation is only distinguishable after this period. This is not in agreement with a study by Nelissen et al. (2013), in which it was found that the fetal growth patterns diverged already at 20 weeks of gestation after the use of two different culture media. It has been suggested that many ART procedures, including certain culture conditions, are associated with fetal growth restriction in early to mid-pregnancy, succeeded by substantial increases in placental size and accelerated fetal growth toward the end of gestation (Bloise et al., 2014). In mice, it was shown that culture medium composition highly determines fetal weight after ART (Delle Piane et al., 2010).

Although speculative, the effect that embryo culture media have on birth weight of newborns could be mediated through a direct effect on the epigenome of the developing preimplantation embryo (van Montfoort et al., 2012). In recent publications, we demonstrated that the use of G5 and HTF resulted in different gene expression profiles in human preimplantation embryos at the blastocyst stage (Kleijkers et al., 2015a; Mantikou et al., 2016). A side-by-side comparison of five human culture media indicated that all had a varying but compromised ability to maintain gene expression and DNA methylation patterns in mouse preimplantation embryos as compared with in vivo-derived embryo (MarketVelker et al., 2010). Alternatively, it could be that embryo culture media affect the ability of preimplantation embryos to properly implant. In such a scenario, the observed lower birth weight, as well as the increased incidence of preterm birth, would be an indirect effect of culture media via disturbed implantation.

It is too early to know whether a difference of 158 grams in birth weight has any clinical significance. However, an inverse association between birth weight and increased rates of coronary heart disease and the related disorders of stroke, 
hypertension and type 2 diabetes during adult life has previously been demonstrated (Barker, 1997). This has led to the Barker or developmental origin of health and disease (DOHaD) hypothesis, which suggests that the fetus is sensitive to its (uterine) environment, and that adaptations of the fetus to its environment have lasting consequences for its development, growth and health. A well-known example of the DOHaD hypothesis is the finding that individuals conceived during the Dutch famine of the second World War have higher rates of coronary heart disease, a more atherogenic lipid profile, disturbed blood coagulation, increased stress responsiveness and are more often obese than unexposed individuals or those exposed during mid- or late gestation, even without an effect on birth weight (Roseboom et al., 2011). It has also been suggested that preimplantation embryos or even gametes adapt to their altering environment in response to parental diet with long-term consequences for health of the future adult (Carone et al., 2010; Fleming et al., 2012; Lucas, 2013).

One of the differences between the embryo culture media is that the G5 medium contains certain amino acids and that the HTF medium completely lacks amino acids. Studies in human and animal embryos have shown that the embryo development in vitro can indeed be improved by the addition of amino acids to the culture medium (Liu and Foote, 1995; Devreker et al., 1998; Lane and Gardner, 1998; Summers and Biggers, 2003). The importance of amino acids during preimplantation embryo development also stems from studies in which mice were given a low protein diet (LPD) only during the period of conception. The offspring had a higher birth weight and blood pressure, even when the embryos were transferred to a pseudo pregnant mouse that did not have the LPD during conception (Watkins et al., 2008; Fleming et al., 2012). We are not aware of any study that has looked specifically at the effect of amino acids in embryo culture media on pregnancy rates, live births or perinatal outcome in humans. Other notable differences in composition are the addition of hyaluronan and lipoic acid to G5. A meta-analysis showed that the use of hyaluronan-enriched transfer media resulted in increased implantation and live birth rates (Bontekoe et al., 2014). Although lipoic acid is thought to improve mouse embryo development via its role as antioxidant (Talebi et al., 2012), no studies have evaluated its effect on pregnancy or live birth rates in humans and no studies have looked at the effect of hyaluronan- or lipoic acid-enrichment of embryo culture media on perinatal outcome. The protein source in the culture media is also an important candidate in this respect, as the quality of it has been shown to vary considerably between batches as well as between manufacturers (Dyrlund et al., 2014; Morbeck et al., 2014b) and the protein source has previously been suggested to influence birth weight (Zhu et al., 2014a). 
It is relevant to note that HTF was widely used for many years in IVF worldwide but has lost significant market share in recent years. Embryologists now seem to prefer other, more 'enriched media', i.e. media that contain additives such as the previously mentioned amino acids. Still, HTF is not completely removed from the market. At the time of study design, 8 out of 13 IVF centers in the Netherlands used HTF medium to culture preimplantation embryos. Based on the Dutch national reports of the IVF results (NVOG), there were no clear differences in ongoing pregnancy rates between centers using HTF medium and the other centers. Two small studies published as abstracts indicated that $G 2$ medium (G2 is an earlier version of $\mathrm{G} 5$ and is no longer on the market) had a higher efficacy than HTF medium (Bisioli et al., 2003; Choi et al., 2004), however, well designed randomized controlled trials comparing these media to more classic media were heretofore lacking. Proper trials to support these assumptions are very much needed according to the good clinical practice guidelines (Mantikou et al., 2013b).

Over 20 other embryo culture media are commercially available. A trial comparing all available culture media simultaneously is simply not possible. Nevertheless, such an essential component of IVF should be treated with the highest level of scrutiny, and there is a need for more well designed randomized trials on media for human embryo culture focusing not only on pregnancy rates but also on birth weight and child health. In addition, the full composition of embryo culture media should be made publically available by the companies that produce them (Summers and Biggers, 2003). Currently the full composition of embryo culture media is available for some culture media, but not all (Morbeck et al., 2014a). The formulations should have a scientific rationale and the introduction of new media into clinical practice should be based on properly conducted RCTs. Companies should also report what studies have been performed to test these media and which endpoints have been analyzed. In our view, the responsibility for proper introduction of embryo culture media with new formulations not only lies with the manufacturers, but also with clinical embryologists that decide to implement these culture media in clinical practice. The legislative background behind IVF culture media is such that there are limited constraints in introducing new culture media, even if they contain new growth factors of unknown effect on pregnancy rates let alone the health of children born. We feel the time has come to radically shift gears in our field and that we should no longer blindly accept new culture media (or other alterations in laboratory or clinical procedures) without first rigorously studying effectiveness and safety not unlike introduction of new drugs or medical devices.

Ongoing pregnancy or live birth rate is generally considered to be the most important outcome after IVF (Land and Evers, 2003; Braakhekke et al., 2014). Our study should raise awareness of the fact that perinatal outcomes potentially are 
just as important and that follow-up procedures should be an integral part of the treatment protocol in every IVF center worldwide. It has been well argued that professionals in the field of reproduction have the moral obligation to set-up (long-term) follow-up studies, and that the liability in this cannot simply be left to the patient by means of 'informed consent' (Dondorp and de Wert, 2011). While a patient could in principle agree with being treated with a technique of unknown safety and effectiveness, the clinician still remains responsible for what he or she does (Dondorp and de Wert, 2011).

In conclusion, we have demonstrated a significant effect of embryo culture media used in IVF on pregnancy outcome and on the birth weight of newborns. This suggests that the millions of human embryos that are cultured in vitro each year are highly sensitive to their environment. These findings should lead to increased awareness, mechanistic studies and legislative adaptations to protect IVF offspring during the first few days of their existence.

\section{Funding}

This project was partly funded by The NutsOhra foundation (Grant 1203-061) and March of Dimes (Grant 6-FY13-153). The NutsOhra foundation and March of Dimes had no role in the development of the study, in the collection, analysis, and interpretation of data, in writing of the manuscript, or in the decision to submit the manuscript for publication. 


\section{Supplementary Tables}

Supplementary Table 1. Clinical characteristics according to treatment cycle.

\begin{tabular}{|c|c|c|c|}
\hline Characteristic & G5 & HTF & P-value \\
\hline \multicolumn{4}{|l|}{ No. of fresh cycles started ${ }^{*}$} \\
\hline Cycle 1 & 417 & 419 & \\
\hline Cycle 2 & $222(53.2)$ & $260(62.1)$ & 0.01 \\
\hline Cycle 3 & $72(17.3)$ & $102(24.3)$ & 0.01 \\
\hline Cycle 4 & $2(0.5)$ & $3(0.7)$ & 1.00 \\
\hline Total & 713 & 784 & \\
\hline \multicolumn{4}{|l|}{ Fertilization procedure } \\
\hline ICSI & $416(58.3)$ & 449 (57.3) & 0.68 \\
\hline No. of fresh embryo transfers ${ }^{\dagger}$ & $663(93.0)$ & 733 (93.5) & 0.76 \\
\hline \multicolumn{4}{|l|}{ No. of fresh cycles with live birth ${ }^{f}$} \\
\hline Cycle 1 & $93(22.3)$ & $76(18.1)$ & 0.14 \\
\hline Cycle 2 & $52(23.4)$ & $46(17.7)$ & 0.14 \\
\hline Cycle 3 & $11(15.3)$ & $13(12.7)$ & 0.66 \\
\hline Cycle 4 & $0(0)$ & $0(0)$ & $\mathrm{n} / \mathrm{a}$ \\
\hline No. of transfers with cryopreserved embryos & $276(38.7)$ & $204(26.0)$ & $<0.001$ \\
\hline \multicolumn{4}{|l|}{ No. of transfers with cryopreserved embryos ${ }^{\ddagger}$} \\
\hline As part of cycle 1 & $206(49.4)$ & $148(35.3)$ & $<0.001$ \\
\hline As part of cycle 2 & $55(24.8)$ & $49(18.8)$ & 0.12 \\
\hline As part of cycle 3 & $15(20.8)$ & $7(6.9)$ & 0.01 \\
\hline As part of cycle 4 & $0(0)$ & $0(0)$ & $\mathrm{n} / \mathrm{a}$ \\
\hline \multicolumn{4}{|c|}{ No. of transfers with cryopreserved embryos leading to a live birth ${ }^{\S}$} \\
\hline As part of cycle 1 & $21(10.2)$ & $19(12.8)$ & 0.50 \\
\hline As part of cycle 2 & $6(10.9)$ & $5(10.2)$ & 1.00 \\
\hline As part of cycle 3 & $1(6.7)$ & $0(0)$ & 1.00 \\
\hline As part of cycle 4 & $\mathrm{n} / \mathrm{a}$ & $\mathrm{n} / \mathrm{a}$ & n/a \\
\hline
\end{tabular}

Data presented as number (\%). Percentages calculated per total number of fresh cycles started. N/A denotes not applicable.

* Percentages calculated per number of fresh cycle 1 started.

${ }^{\dagger}$ One woman in each group had two embryo transfers using fresh and vitrified oocytes.

${ }^{\mathrm{f}}$ Percentage calculated per number of fresh cycles started.

${ }^{\S}$ Percentage calculated per number of embryo transfers with cryopreserved embryos. 


\section{Chapter 2}

Supplementary Table 2. The effect of culture medium on birth weight controlled for potential birth weight confounders.

\begin{tabular}{lccc}
\hline Variable & \multicolumn{1}{c}{$\beta^{*}$} & $95 \% \mathrm{Cl}^{*}$ & P-value \\
\hline Culture medium (G5 versus HTF) & -116 & -212 to -20 & 0.02 \\
Child's gender (male versus female) & 144 & 47 to 241 & 0.004 \\
Gestational age at birth (per week) & 188 & 159 to 218 & $<0.001$ \\
Pregnancy complications (yes versus no) & -23 & -158 to 111 & 0.73 \\
No. of transferred embryos (1 versus $>1$ ) & -30 & -128 to 69 & 0.56 \\
Embryo transfer (fresh versus cryo) & 70 & -61 to 200 & 0.29 \\
Parity (primiparous versus multiparous) & 134 & 28 to 240 & 0.01 \\
Maternal height (per cm) & 9 & 1 to 16 & 0.03 \\
Maternal weight (per kg) & 5 & 1 to 10 & 0.01 \\
Maternal smoking (<10 versus $\geq 10$ cig/day)* & -262 & -420 to -105 & 0.001 \\
Paternal height (per cm) & 2 & -5 to 9 & 0.55 \\
Paternal weight (per kg) & 2 & -2 to 6 & 0.27 \\
\hline
\end{tabular}

$\beta$, regression coefficient; $\mathrm{Cl}$, confidence interval; cig/day, cigarettes per day. 


\section{CHAPTER 3}

\section{IVF culture medium affects postnatal weight in humans during the first 2 years of life}

Sander H.M. Kleijkers, Aafke P.A. van Montfoort, Luc J.M. Smits, Wolfgang Viechtbauer, Tessa J. Roseboom, Ewka C.M. Nelissen, Edith Coonen, Josien G. Derhaag, Lobke Bastings, Inge E.L. Schreurs, Johannes L.H. Evers, John C.M. Dumoulin

Human Reproduction 2014;29(4):661-669. 


\section{Abstract}

Study question: Is postnatal growth during the first 2 years of life in IVF singletons affected by type of medium used for culturing human embryos during an IVF treatment?

Summary answer: The in vitro culture of human embryos in medium from Cook resulted in singletons with a lower weight during the first 2 years of life compared with singletons born after embryo culture in medium from Vitrolife.

What is known already: In a previous study, we reported that type of medium used for culturing human IVF embryos during the first few days after fertilization until fresh embryo transfer significantly affects fetal growth and consequently birth weight of the resulting singletons.

Study design, size, duration: From July 2003 to December 2006, a total of 1432 IVF treatment cycles with fresh embryo transfer were randomly allocated to have all embryos cultured in medium from Vitrolife $A B(n=715)$ or from Cook $(n=717)$. Two years after delivery, questionnaires were sent to the parents of all children to collect data about weight, height and head circumference around 1, 2, 3, 4, 6, 7.5, $9,11,14,18$, and 24 months of age. Those points of measurements are part of the children's health program at municipal infant welfare centers in the Netherlands, in which children are routinely measured by health professionals unaware of this study.

Participants/materials, setting, methods: Patients requiring donor oocytes or applying for PGD were excluded from the study. From the 294 live born singletons that fulfilled our inclusion criteria, 29 were lost to follow-up. The remaining 265 singletons (Cook group: 117, Vitrolife group: 148) were included in the analysis. Data analysis included linear regression, to compare cross-sectionally weight standard deviation score (SDS), height SDS and head circumference, and the first order Berkey-Reed model for a longitudinal analysis of the growth data.

Main results and the role of chance: Singletons in the Vitrolife group were heavier during the first 2 years of life compared with singletons in the Cook group. Cross-sectional analyses showed that adjusted weight SDS differed between groups at $1(0.35 \pm 0.14, P=0.010), 2(0.39 \pm 0.14, P=0.006), 3(0.35 \pm 0.14, P=$ $0.011), 4(0.30 \pm 0.13, P=0.020), 11(0.28 \pm 0.13, P=0.036), 14(0.32 \pm 0.13, P=$ $0.014)$, and $24(0.39 \pm 0.15, P=0.011)$ months of age, while adjusted height SDS was only significantly different at $1(0.21 \pm 0.11, P=0.048)$ month of age. Head circumference was similar between the two groups at all ages. Longitudinal analyses showed that both postnatal weight $(P=0.005)$ and height $(P=0.031)$ 
differed between the groups throughout the first 2 years of life, while the growth velocity was not significantly different between the two groups.

Limitations, reasons for caution: Factors that might influence postnatal growth were included in the analysis however it was not possible to include all such factors, for example childhood diseases or nutrition, as this information was not available.

Wider implications of the findings: The effect of culture medium during the first few days after fertilization on prenatal growth and birth weight persists during the first 2 years of life. This suggests that the human embryo is sensitive to its very early environment, and that the culture medium used in IVF may have lasting consequences. Further monitoring of the long-term growth, development and health of IVF children is therefore warranted.

Study funding/competing interest(s): W.V. was funded with an unrestricted research grant from the Stichting Fertility Foundation. The authors declare no conflict of interest.

Trial registration number: Not applicable. 


\section{Introduction}

The first child conceived by IVF was born 35 years ago (Steptoe and Edwards, 1978). Since then, the use of assisted reproduction technologies (ART) has increased substantially. In 2009, the percentage of children conceived with in vitro techniques ranged from $0.6 \%$ up to $4.5 \%$ of the national births in Europe (Ferraretti et al., 2013).

IVF singletons have a significantly increased risk of perinatal mortality, preterm birth, small for gestational age, low birth weight, and birth defects (Helmerhorst et al., 2004; Jackson et al., 2004; Rimm et al., 2004; McDonald et al., 2009; Pandey et al., 2012) compared with naturally conceived singletons. Both patient-related factors, for example older age of the mother, time to pregnancy, and subfertility of the parents, and specific aspects of ART, for example hormonal stimulation and culture conditions, may be risk factors for the adverse perinatal outcome in IVF children (Pinborg et al., 2013).

Our group showed that the culture medium used during an IVF treatment affects birth weight (Dumoulin et al., 2010; Nelissen et al., 2012). The onset of the growth differentiation was already evident during the second trimester of pregnancy, as a more advanced fetus was seen in the Vitrolife group compared with the Cook group at 20 weeks of gestation, based on the differences between gestational age calculated by dating formulas using fetal biparietal diameter and the actual gestational age (Nelissen et al., 2013). It is important to monitor the postnatal growth of these children to investigate whether this effect of culture medium disappears, is still present, or increases later in life.

The aim of our present study was to compare postnatal weight, height and head circumference at several time points (cross-sectional analysis) and postnatal growth (longitudinal analysis) during the first 2 years of life in the same singletons as our previous study.

\section{Material and methods}

\section{Study design}

At the Maastricht University Medical Centre, The Netherlands, a total of 1432 IVF treatment cycles with fresh embryo transfer in the period from July 2003 to December 2006 were randomly allocated to culture in either medium from Cook (K-SICM, Brisbane, Australia; $\mathrm{n=717)}$ ) or from Vitrolife AB (G1.3 , Göteborg, Sweden; $n=715)$. Both culture media were commercially available and widely 
used. The media from both suppliers were ready-to-use and supplemented with 5 $\mathrm{mg} / \mathrm{mL}$ human serum albumin. Laboratory technicians strictly alternated between the two culture media for each cycle and this allocation was concealed from the clinicians involved in scheduling the order of ovum retrieval on a certain day, as well as from the patients. The alternate use of two culture media was part of an internal quality monitoring system that had been introduced to enable the identification of suboptimal batches of a particular medium. Besides type of culture medium, all other procedures in the IVF treatment, for example ovarian stimulation, fertilization, culture, and embryo transfer procedures, were similar in both groups, as have been described earlier (Dumoulin et al., 2010).

For the present study, all singleton live births after fresh embryo transfer were included. Patients requiring donor oocytes or applying for PGD were excluded from the study. In case couples had more than one child during the study, only data from the first singleton pregnancy were included in the analysis. This resulted in 126 live born singletons in the Cook group and 168 singletons in the Vitrolife group (Nelissen et al., 2012). All participating couples gave written informed consent for the use of their data. The local medical ethics committee approved the collection of data for quality monitoring purposes as part of our IVF treatment protocol.

\section{Study population and data collection}

During the study period, only patients with a BMI of $<30 \mathrm{~kg} / \mathrm{m}^{2}$ and $\leq 40$ years of age, with the exception of a few cases, were admitted to our IVF program. Parental weight, height and smoking habits were recorded at the start of each IVF treatment cycle. After delivery, the obstetricians and midwives were contacted to obtain information on pregnancy outcome. Two years after delivery, questionnaires were sent to the parents of all children to collect data about weight, height and head circumference around 1, 2, 3, 4, 6, 7.5, 9, 11, 14, 18, and 24 months of age. Those points of measurements are part of the children's health program at municipal infant welfare centers in the Netherlands, in which children are routinely measured by health professionals unaware of this study. Head circumference was only measured until the age of 11 months in most cases. Postnatal weight and height measurements are expressed as standard deviation score (SDS), normalized for age and gender, and were calculated by using the online TNO growth calculator, which is based on the data from the Fifth National Growth Study (TNO, 2010).

\section{Statistical analysis}

Cross-sectional differences in weight, height and head circumference between study groups were tested by use of the Student's t-test. Adjusted differences were 
analyzed by use of linear multivariable regression analysis, while controlling for the following covariates: parental characteristics (height, weight, smoking habits and age), parity, gestational age at birth, and gender. In case of weight SDS and height SDS, the covariate gender was omitted, as the SDS is normalized for gender.

To study whether the growth velocity of children from the Cook group was different from that of children from the Vitrolife group during the first 2 years of life, a variety of different growth models were applied on the pooled growth data from both groups. The goal was to find a (parametric) model that adequately captures the individual and overall growth trends. In particular, the Count, (first and second order) Berkey-Reed, and several non-linear models (Jenss-Bayley, Gompertz, four-parameter logistic) were examined for their fitness (Jenss and Bayley, 1937; Berkey and Reed, 1987; Pinheiro and Bates, 2000; Hauspie and Molinari, 2004). In all cases, a mixed-effects model was used with random effects corresponding to all model variables with an unstructured variance-covariance matrix. Analyses were conducted with R (version 2.15.2) using the "nlme" package (Pinheiro and Bates, 2000).

Among the various models considered, the first order Berkey-Reed model (Berkey and Reed, 1987) was given preference, for the following reasons. First, it is the model that fits the data best. Second, it is a linear model and therefore easier to fit than the non-linear models. In addition, the model automatically incorporates the gestation length into the model, as the age variable is counted starting at conception (which is exactly known in these cases). The basic model is described by:

$y_{i j}=\beta_{0 i}+\beta_{1 i} \operatorname{age}_{i j}+\beta_{2 i} \log \left(\right.$ age $\left._{i j}\right)+\beta_{3 i}\left(\text { age }_{i j}\right)^{-1}+\beta_{4}$ medium $_{i}+e_{i j}$

where $y_{i j}$ denotes the $j$ th measurement for the $i$ th individual, age $e_{i j}$ denotes the age of measurement (with age 0 corresponding to conception), and $\log \left(\mathrm{age}_{\mathrm{ij}}\right)$ and $\left(\text { age }_{\mathrm{ij}}\right)^{-1}$ are the corresponding log and inverse transformed values of age $\mathrm{e}_{\mathrm{ij}}$.

To avoid very small coefficients, the age variable was coded in years. Two-sided $P$ values of $<0.05$ were considered to reflect statistical significance.

\section{Results}

Of the 294 live born singletons (Cook group: 126, Vitrolife group: 168) that fulfilled our inclusion criteria, 29 were lost to postnatal follow-up (Cook group: 9, Vitrolife group: 20), because parents declined to participate or could not be traced. The remaining 265 (Cook group: 117 (92.9\%), Vitrolife group: 148 (88.1\%)) were included in the analysis. Parental and cycle characteristics are presented in Table 1. 
Table 1. Parental and cycle characteristics in a study of the effect of IVF culture medium on postnatal growth during the first 2 years of life

\begin{tabular}{|c|c|c|}
\hline Characteristic & Vitrolife group $(n=148)$ & Cook group $(n=117)$ \\
\hline \multicolumn{3}{|l|}{ Primary indication for IVF treatment } \\
\hline Tubal factor & $15(10.1)$ & $16(13.7)$ \\
\hline Male factor & $91(61.5)$ & $74(63.2)$ \\
\hline Unexplained & $35(23.6)$ & $23(19.7)$ \\
\hline Other & $7(4.7)$ & $4(3.4)$ \\
\hline Duration of subfertility (years) & $3.36 \pm 1.7$ & $3.48 \pm 1.9$ \\
\hline Primary subfertility & $114(77.0)$ & $86(73.5)$ \\
\hline Cycles with ICSI & $93(62.8)$ & $75(64.1)$ \\
\hline Cycles with transfer on Day 2 & $114(77.0)$ & $87(74.4)$ \\
\hline Cycles with transfer on Day 3 & $34(23.0)$ & $30(25.6)$ \\
\hline Single embryo transfer (SET) & $74(50.0)$ & $69(59.0)$ \\
\hline \multicolumn{3}{|l|}{ Maternal characteristics } \\
\hline Age (years) & $32.3 \pm 4.0$ & $32.6 \pm 3.6$ \\
\hline Age $>=38$ years & $10(6.8)$ & $8(6.8)$ \\
\hline Height $(\mathrm{cm})$ & $169.4 \pm 6.0$ & $167.8 \pm 7.4$ \\
\hline Weight (kg) & $69.9 \pm 10.2$ & $67.6 \pm 10.1$ \\
\hline Smoking >= 10 cigarettes/day & $21(14.2)$ & $16(13.7)$ \\
\hline \multicolumn{3}{|l|}{ Paternal characteristics } \\
\hline Age (years) & $35.2 \pm 5.7$ & $35.7 \pm 4.9$ \\
\hline Height (cm) & $182.3 \pm 8.1$ & $181.2 \pm 7.4$ \\
\hline Weight (kg) & $86.9 \pm 14.4$ & $83.2 \pm 11.0$ \\
\hline Smoking >= 10 cigarettes/day & $25(16.9)$ & $22(18.8)$ \\
\hline
\end{tabular}

Data are presented as $\mathrm{n}(\%)$ or mean \pm SD. No statistical comparisons between groups were performed.

\section{Cross-sectional analysis of postnatal weight, height and head circumference}

Postnatal weight and height measurements were expressed as SDS normalized for age and gender. The weight SDS, height SDS, and head circumference measurements of the singletons are presented in Table 2. Weight SDS was significantly lower in the Cook group compared with the Vitrolife group at all ages. The adjusted mean differences in weight SDS were significant at 1, 2, 3, 4, 11, 14, and 24 months of age. Height SDS was significantly greater in the Vitrolife group compared with the Cook group at 1, 3, 4, 6, 9, 14, and 24 months of age. However, adjusted mean differences in height SDS were only significant at 1 month of age. Head circumference differences and adjusted mean differences were not significant between groups at any of the ages. 


\section{Chapter 3}

Table 2. Postnatal head circumference and standard deviation score (SDS) for weight and height.

\begin{tabular}{|c|c|c|c|c|c|}
\hline Characteristic & $\begin{array}{l}\text { Vitrolife } \\
\text { group }\end{array}$ & $\begin{array}{l}\text { Cook } \\
\text { group }\end{array}$ & P-value & $\begin{array}{l}\text { Adjusted mean } \\
\text { difference }\end{array}$ & P-value \\
\hline \multicolumn{6}{|l|}{ Weight SDS } \\
\hline 1 month & $0.23 \pm 0.10$ & $-0.13 \pm 0.10$ & 0.013 & $0.35 \pm 0.14$ & 0.010 \\
\hline 2 months & $0.49 \pm 0.10$ & $0.06 \pm 0.11$ & 0.004 & $0.39 \pm 0.14$ & 0.006 \\
\hline 3 months & $0.53 \pm 0.09$ & $0.10 \pm 0.11$ & 0.003 & $0.35 \pm 0.14$ & 0.011 \\
\hline 4 months & $0.60 \pm 0.09$ & $0.21 \pm 0.10$ & 0.004 & $0.30 \pm 0.13$ & 0.020 \\
\hline 6 months & $0.48 \pm 0.10$ & $0.15 \pm 0.09$ & 0.019 & $0.24 \pm 0.13$ & NS \\
\hline 7.5 months & $0.44 \pm 0.10$ & $0.13 \pm 0.09$ & 0.025 & $0.23 \pm 0.14$ & NS \\
\hline 9 months & $0.38 \pm 0.10$ & $0.09 \pm 0.10$ & 0.042 & $0.18 \pm 0.14$ & NS \\
\hline 11 months & $0.34 \pm 0.09$ & $-0.02 \pm 0.10$ & 0.008 & $0.28 \pm 0.13$ & 0.036 \\
\hline 14 months & $0.18 \pm 0.09$ & $-0.22 \pm 0.09$ & 0.002 & $0.32 \pm 0.13$ & 0.014 \\
\hline 18 months & $0.02 \pm 0.12$ & $-0.35 \pm 0.10$ & 0.018 & $0.26 \pm 0.15$ & NS \\
\hline 24 months & $0.07 \pm 0.10$ & $-0.43 \pm 0.12$ & 0.001 & $0.39 \pm 0.15$ & 0.011 \\
\hline \multicolumn{6}{|l|}{ Height SDS } \\
\hline 1 month & $-0.17 \pm 0.08$ & $-0.44 \pm 0.08$ & 0.021 & $0.21 \pm 0.11$ & 0.048 \\
\hline 2 months & $-0.05 \pm 0.13$ & $-0.32 \pm 0.12$ & NS & $0.07 \pm 0.17$ & NS \\
\hline 3 months & $0.17 \pm 0.08$ & $-0.13 \pm 0.11$ & 0.019 & $0.19 \pm 0.11$ & NS \\
\hline 4 months & $0.34 \pm 0.12$ & $-0.10 \pm 0.10$ & 0.006 & $0.26 \pm 0.15$ & NS \\
\hline 6 months & $0.24 \pm 0.09$ & $-0.01 \pm 0.08$ & 0.041 & $0.13 \pm 0.11$ & NS \\
\hline 7.5 months & $0.18 \pm 0.13$ & $-0.06 \pm 0.12$ & NS & $0.09 \pm 0.17$ & NS \\
\hline 9 months & $0.21 \pm 0.08$ & $-0.08 \pm 0.09$ & 0.019 & $0.15 \pm 0.12$ & NS \\
\hline 11 months & $0.23 \pm 0.09$ & $0.03 \pm 0.10$ & NS & $0.09 \pm 0.12$ & NS \\
\hline 14 months & $0.24 \pm 0.08$ & $-0.02 \pm 0.09$ & 0.043 & $0.16 \pm 0.12$ & NS \\
\hline 18 months & $0.19 \pm 0.10$ & $-0.03 \pm 0.10$ & NS & $0.10 \pm 0.13$ & NS \\
\hline 24 months & $0.25 \pm 0.10$ & $-0.14 \pm 0.11$ & 0.010 & $0.27 \pm 0.14$ & NS \\
\hline \multicolumn{6}{|c|}{ Head circumference $(\mathrm{cm})$} \\
\hline 1 month & $37.20 \pm 0.14$ & $36.92 \pm 0.14$ & NS & $0.25 \pm 0.17$ & NS \\
\hline 2 months & $38.99 \pm 0.14$ & $38.80 \pm 0.12$ & NS & $0.14 \pm 0.16$ & NS \\
\hline 3 months & $40.32 \pm 0.13$ & $40.31 \pm 0.14$ & NS & $0.00 \pm 0.16$ & NS \\
\hline 4 months & $41.76 \pm 0.13$ & $41.63 \pm 0.12$ & NS & $0.13 \pm 0.16$ & NS \\
\hline 6 months & $43.44 \pm 0.13$ & $43.40 \pm 0.13$ & NS & $-0.02 \pm 0.16$ & NS \\
\hline 7.5 months & $44.50 \pm 0.13$ & $44.39 \pm 0.13$ & NS & $0.11 \pm 0.16$ & NS \\
\hline 9 months & $45.39 \pm 0.14$ & $45.27 \pm 0.14$ & NS & $0.02 \pm 0.17$ & NS \\
\hline 11 months & $46.26 \pm 0.14$ & $46.13 \pm 0.13$ & NS & $0.06 \pm 0.17$ & NS \\
\hline
\end{tabular}

Data are presented as mean \pm standard error (SE). Unadjusted mean differences are calculated using the Student's t-test and adjusted mean differences using linear multivariable regression analysis, while controlling for the following covariates: parental characteristics (height, body weight, smoking habits and age), parity, gestational age at birth and gender. In case of weight SDS and height SDS, the covariate gender was left out, as the SDS is normalized for gender. 
Longitudinal analysis of postnatal weight and height

To compare the overall growth trajectories of the two groups, a longitudinal analysis was performed. The growth trajectories were constructed by using the first order Berkey-Reed model with the data at 1, 2, 3, 4, 6, 7.5, 9, 11, 14, 18, and 24 months of age from each individual child.

For postnatal weight, the regression coefficients and significance levels of four different Berkey-Reed models are presented in Table 3. Model 1 is the basic model and shows a difference of 216 grams $(P=0.002)$ between the groups, which indicates that during the first 2 years of life, children in the Vitrolife group were heavier compared with children in the Cook group. After addition of covariates, a difference of 188 grams $(P=0.005)$ remained between the groups (model 2). Besides type of culture medium, maternal height $(P=0.003)$, parity $(P=$ $0.003)$ and child's gender $(P<0.001)$ are significantly associated with postnatal weight. To study the effect of culture medium on the velocity of weight gain in the children, the interaction variable culture medium $x$ age was included (model 3 ). The growth trajectories do not diverge significantly from each other $(\beta=0.161, P$ $=0.062$ ), which indicates that children from the Vitrolife group do not gain weight at a faster rate than children from the Cook group. Model 4 shows that maternal weight $(P<0.001)$ and paternal height $(P=0.016)$ are significantly associated with the rate of postnatal weight gain. 


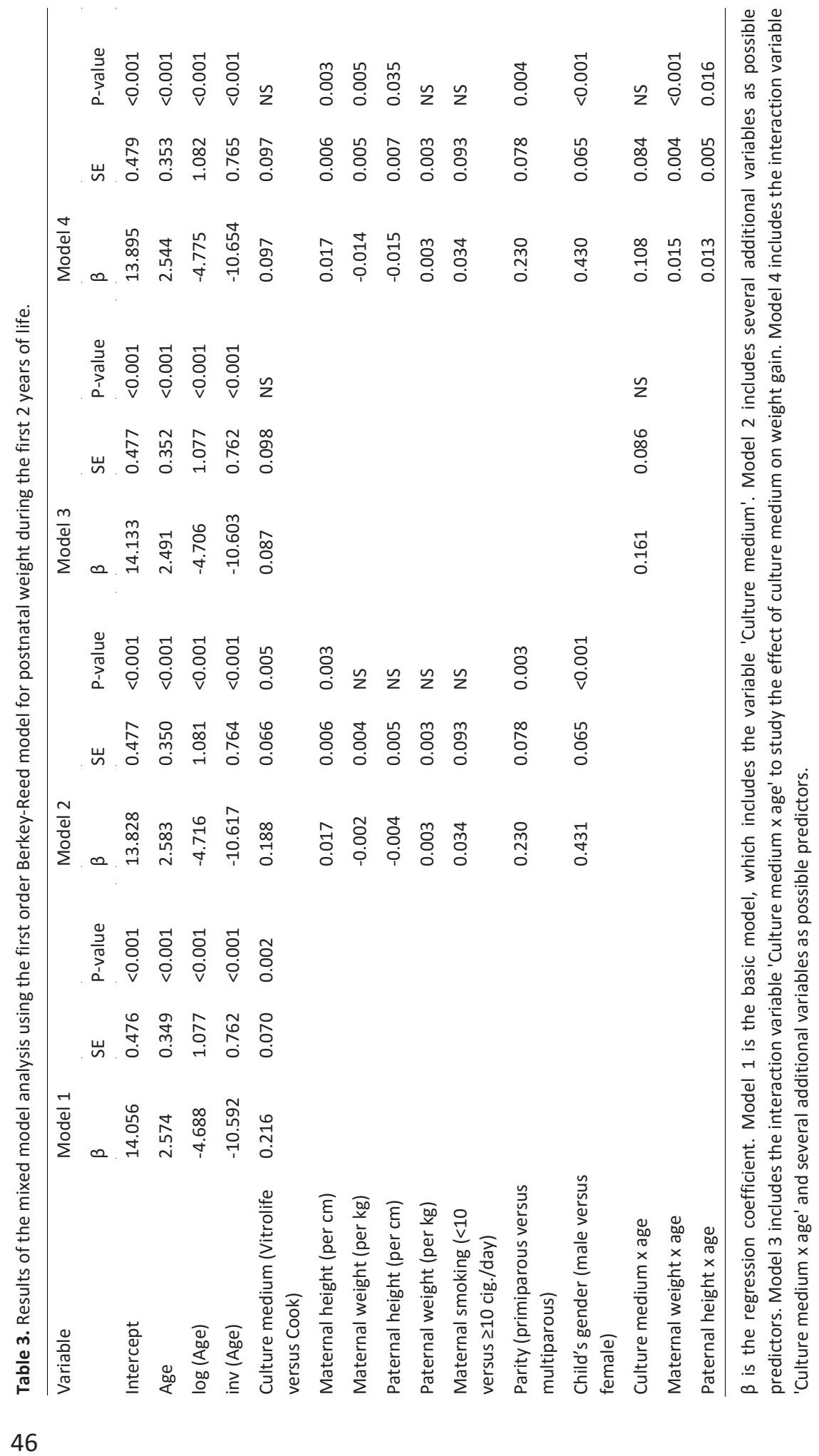


IVF culture medium affects human postnatal weight

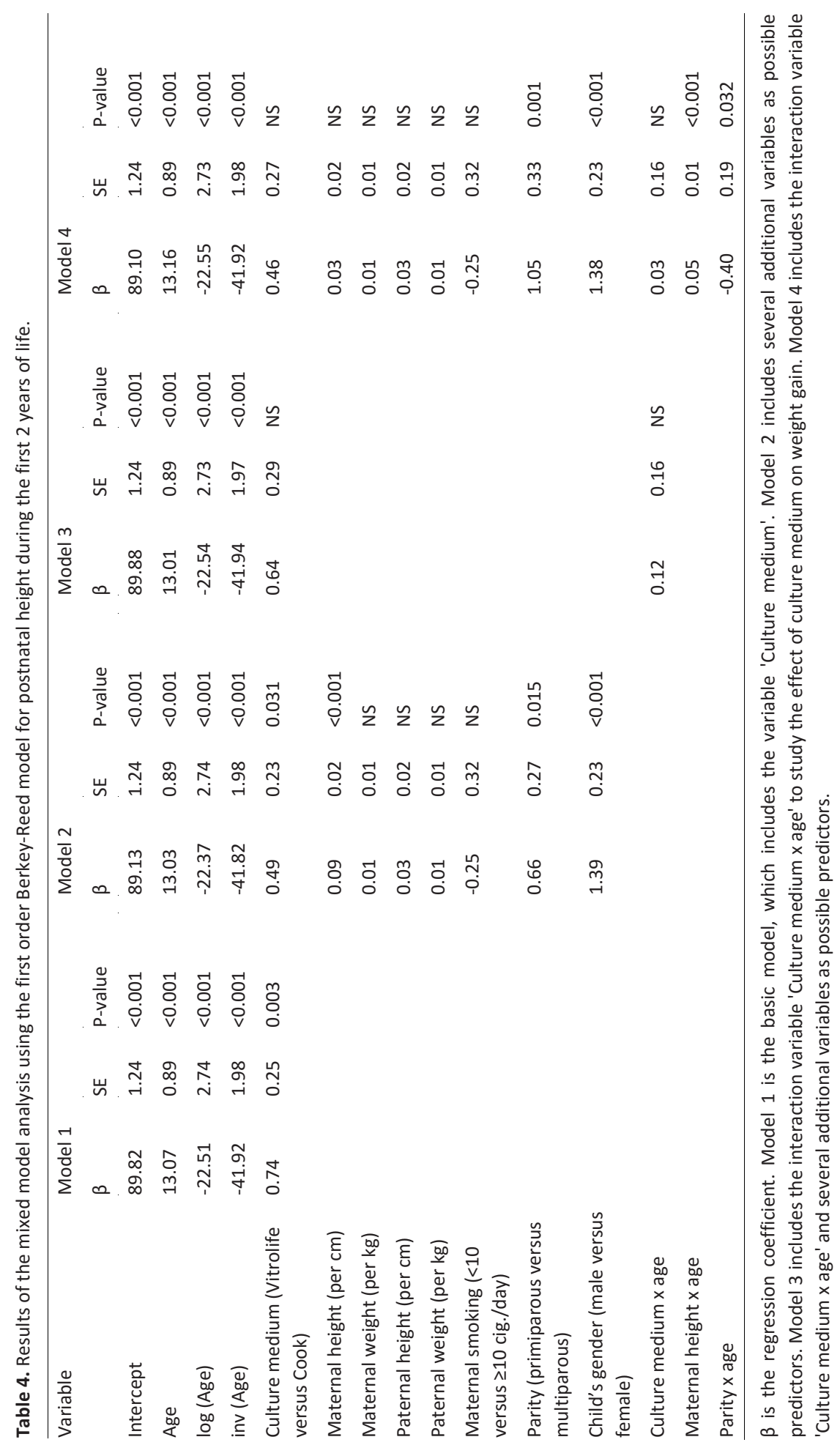


For postnatal height, the regression coefficients and significance levels are presented in Table 4. Model 1 is the basic model and shows a difference of 0.74 $\mathrm{cm}(P=0.003)$ between the groups, with taller children in the Vitrolife group compared with children in the Cook group. After addition of covariates, a difference of $0.49 \mathrm{~cm}(P=0.031)$ remained between the groups (model 2$)$. Besides type of culture medium, maternal height $(P<0.001)$, parity $(P=0.015)$ and child's gender $(P<0.001)$ are significantly associated with postnatal height. The interaction variable culture medium $x$ age was included in model 3 to study the effect of culture medium on the velocity of height gain. The growth trajectories do not diverge significantly from each other $(\beta=0.12, P=0.466)$, which indicates that children from the Vitrolife group do not gain height at a faster rate than children from the Cook group. In model 4 is shown that maternal height $(P<0.001)$ and parity $(P=0.032)$ are significantly associated with the rate of postnatal height gain.

The average growth trajectories for the children from the two culture medium groups are illustrated in Figure 1 for postnatal weight and in Figure 2 for postnatal height. Those are based on the models which include the interaction variable culture medium $x$ age, and are described by:

$y_{i j}=\beta_{0 i}+\beta_{1 i} a e_{i j}+\beta_{2 i} \log \left(\right.$ age $\left._{i j}\right)+\beta_{3 i}\left(\text { age }_{i j}\right)^{-1}+\beta_{4}$ medium $_{i}+\beta_{5}$ age $_{i j} x$ medium $_{i}+e_{i j}$.

Based on these models, the predicted average weight at approximately 4 weeks after birth is $3.99 \mathrm{~kg}$ (95\% confidence interval $(\mathrm{Cl}) 3.89-4.10 \mathrm{~kg}$ ) in the Cook group and $4.21 \mathrm{~kg}(95 \% \mathrm{Cl} 4.12-4.30 \mathrm{~kg})$ in the Vitrolife group and at the age of 2 years the predicted average weights are $12.32 \mathrm{~kg}(95 \% \mathrm{Cl} 12.05-12.59 \mathrm{~kg})$ and $12.85 \mathrm{~kg}$ (95\% Cl 12.61-13.08 kg), respectively. The predicted average height at 4 weeks after birth is $53.2 \mathrm{~cm}(95 \% \mathrm{Cl} 52.8-53.6 \mathrm{~cm})$ in the Cook group and $53.9 \mathrm{~cm}(95 \% \mathrm{Cl}$ 53.6-54.2 $\mathrm{cm}$ ) in the Vitrolife group and at the age of 2 years the predicted average heights are $87.4 \mathrm{~cm}(95 \% \mathrm{Cl} 86.8-88.0 \mathrm{~cm})$ and $88.4 \mathrm{~cm}(95 \% \mathrm{Cl} 87.9-88.9$ $\mathrm{cm})$, respectively. 


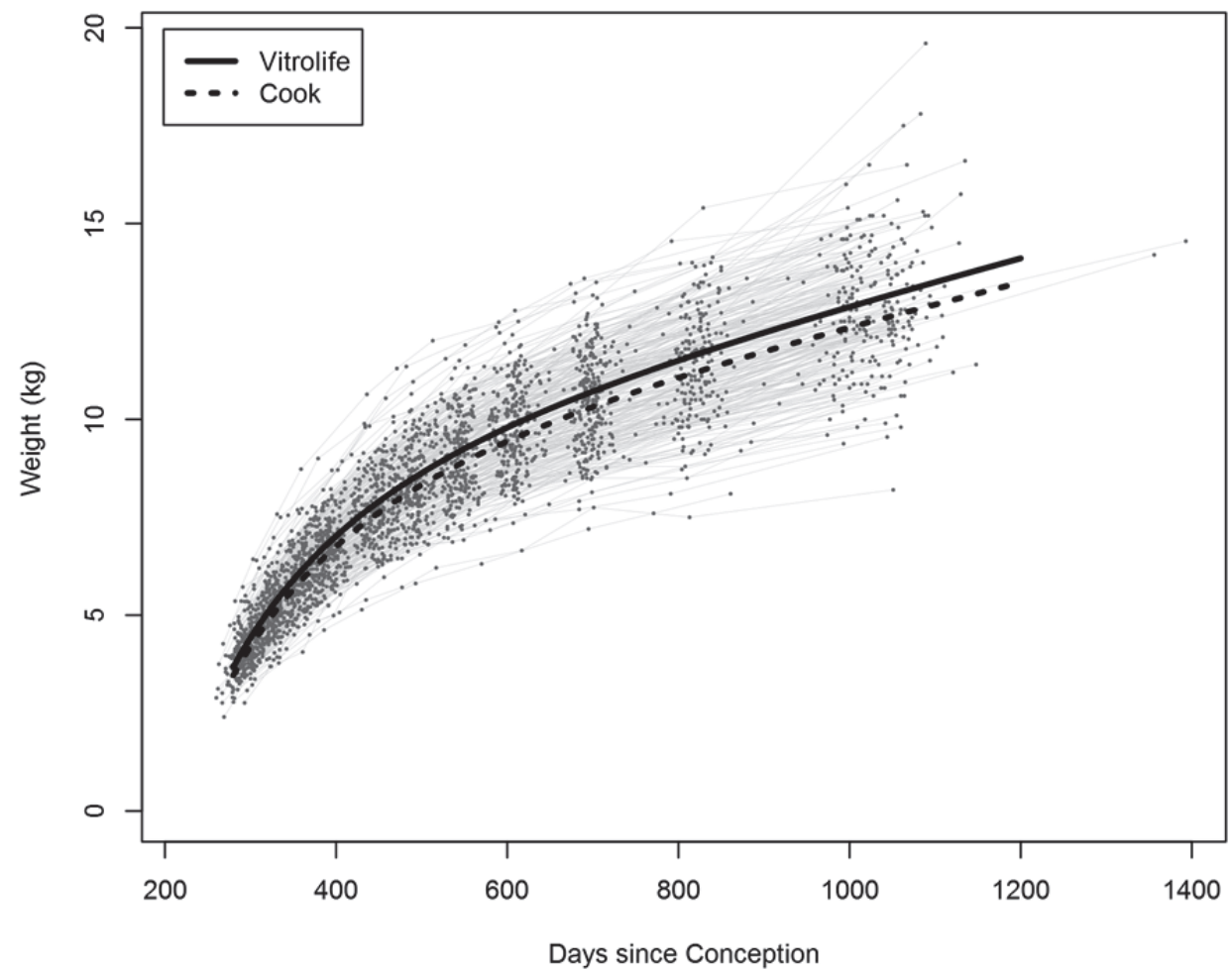

Figure 1. The average weight trajectories for the children from the culture medium groups superimposed on the observed individual trajectories during the first 2 years of life. 


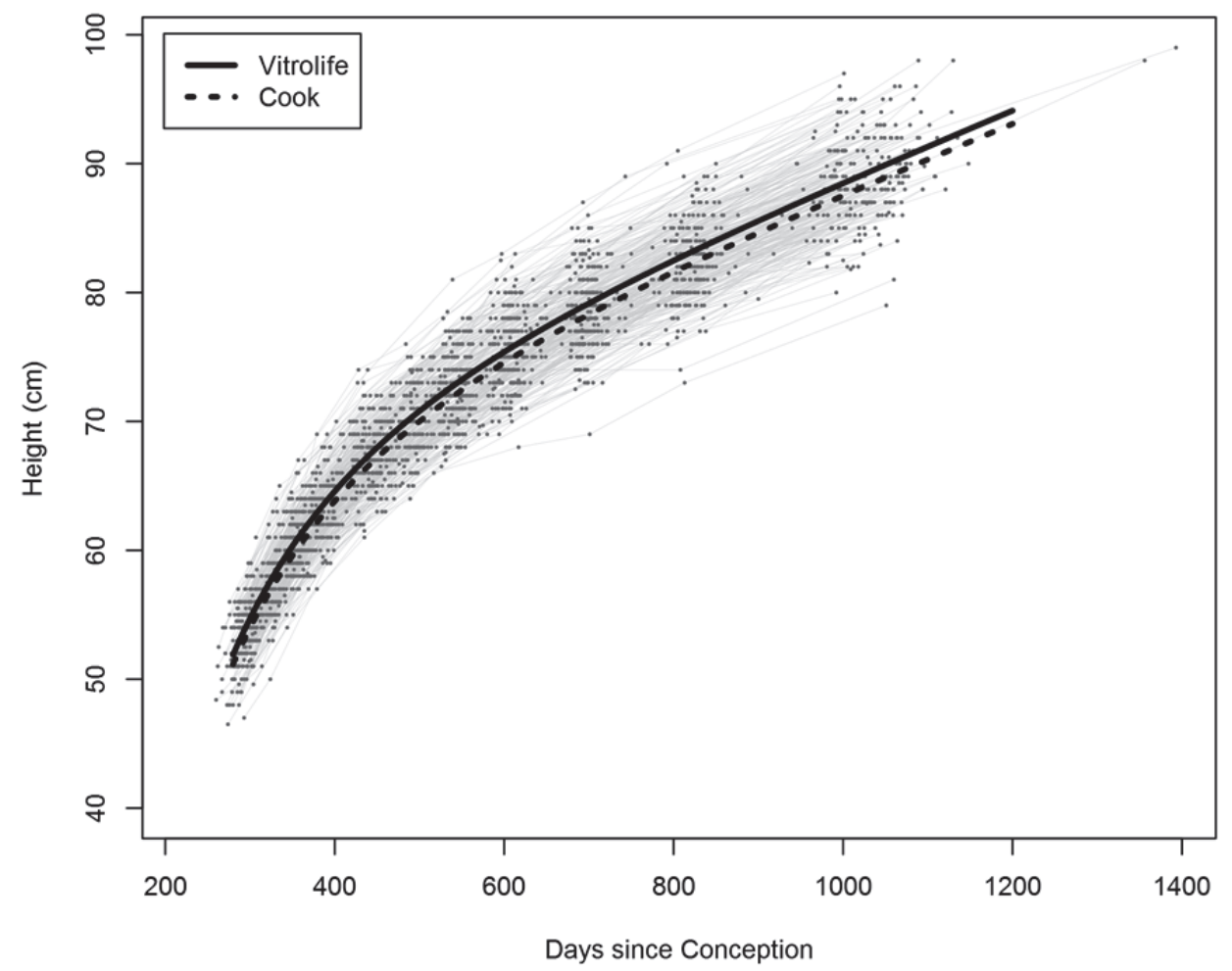

Figure 2. The average height trajectories for the children from the culture medium groups superimposed on the observed individual trajectories during the first 2 years of life.

\section{Discussion}

The main finding of this study is that in vitro culture of human embryos in medium from Cook resulted in singletons with a lower weight during the first 2 years of life compared with singletons born after embryo culture in medium from Vitrolife. This indicates that the effect of culture medium on fetal development and birth weight (Dumoulin et al., 2010; Nelissen et al., 2012; Nelissen et al., 2013) persists during the first 2 years of life.

Since the publication of our previous study (Dumoulin et al., 2010; Nelissen et al., 2012), there have been several other studies published on the effect of culture medium on birth weight. Several of these observational studies, in which other culture media were compared, found no differences in birth weight between the culture medium groups (Eaton et al., 2012; Vergouw et al., 2012; Carrasco et al., 2013; Lin et al., 2013). However, in these studies the culture media have been used in consecutive time periods. Carrasco et al. performed a small randomized study ( $n=98$ ), comparing newer versions of the Cook and Vitrolife media (Carrasco 
et al., 2013). Although, the average birth weight was lower in the Cook group compared with the Vitrolife group, they found no significant difference in birth weight between the groups. Recently, Eskild et al. published a large study including the birth weights of 2435 singletons after the use of 3 different culture media and compared those with the birth weights of offspring from spontaneous conceptions ( $n=698,359)$ during the corresponding time periods (Eskild et al., 2013). They found a significant effect of culture medium on birth weight of the offspring, also after adjustment for changes in birth weight after spontaneous pregnancies. To our knowledge, there are no prior studies on the effect of culture medium on postnatal growth in humans.

This study allows a valid comparison of the effect of two commercially available embryo culture media on postnatal growth in IVF children, since exactly the same ovarian stimulation, fertilization, culture and embryo transfer procedures were applied in each group. Furthermore, several factors that might influence postnatal growth were included in the analysis, for example parental weight and height are shown to be contributors to postnatal child growth (Blair et al., 2004; Griffiths et al., 2007; Hindmarsh et al., 2008; Mesman et al., 2009). However, it was not possible to include all factors, e.g. childhood diseases or nutrition, as this information was not available.

Although the components in the media from this study are known, the exact concentrations of the different components were not disclosed by the suppliers. Therefore, it would be speculative to relate the differences found to specific components in the culture media.

Since the first use of IVF, there has been a major interest in the health of IVF children. Several studies have investigated the growth of children born after IVF. The vast majority of these studies did not find differences in postnatal weight and height between IVF children and naturally conceived children (Ceelen et al., 2009; Basatemur et al., 2010; Beydoun et al., 2010; Lee et al., 2010; Woldringh et al., 2011). However, Ceelen et al. found that growth velocity was higher in IVF children compared with naturally conceived children and that rapid weight gain during early childhood was associated with increased cardiovascular risks in IVF children (Ceelen et al., 2009). Furthermore, it was found that healthy IVF children and adolescents displayed systemic and pulmonary vascular dysfunction, which appeared to be related to the IVF procedure itself (Scherrer et al., 2012).

It is too early to know if the differences that we have found in this study have any clinical significance. However, the findings are in line with the growing body of evidence that suggests that the fetus is sensitive to the uterine environment, and that adaptations of the fetus to its environment have lasting consequences for its 
development, growth and health. This was first proposed by Barker, who found an association between low birth weight and increased rates of coronary heart disease and the related disorders of stroke, hypertension and type 2 diabetes during adult life (Barker, 1997). In the Dutch famine study it has been observed that adults who had been exposed to famine in early gestation had higher rates of coronary heart disease, a more atherogenic lipid profile, disturbed blood coagulation, increased stress responsiveness and were more often obese than mid-gestation, late-gestation and non-exposed individuals (Roseboom et al., 2006). Furthermore, it has been suggested that, even during the periconceptional period, gametes and preimplantation embryos adapt to their environment with long-term health consequences (Steegers-Theunissen et al., 2013). A maternal low-protein diet given to pregnant mice or rats exclusively during the preimplantation period resulted in offspring with increased weight, sustained hypertension, and abnormal anxiety-related behavior (Kwong et al., 2000; Watkins et al., 2008). Maternal under-nutrition around the periconceptional and preimplantation period affects fetal and placental growth in sheep (MacLaughlin et al., 2005). Fernandez-Gonzalez et al. showed that female mice aged 31 to 70 weeks, that had been cultured during the preimplantation period in medium with serum, were significantly heavier compared with female mice cultured as an embryo in medium without serum (Fernandez-Gonzalez et al., 2004). Furthermore, mice sacrificed at 20 months of age showed large organs when cultured in medium with serum during the preimplantation period, as well as long-term neurodevelopmental and behavioral effects (Ecker et al., 2004; Fernandez-Gonzalez et al., 2004).

Several animal studies have shown that culture medium affects gene expression (van Montfoort et al., 2012). Rinaudo and Schultz (2004) compared global patterns of gene expression in mouse blastocysts cultured in either Whitten's medium or KSOM/AA with that of blastocysts that developed in vivo. Culture in Whitten's medium affected the expression of 114 genes, while only 29 genes were differently expressed after culture in KSOM/AA compared with in vivo counterparts. A side-by-side comparison of five commercial culture media showed that all five media had a varying but compromised ability to maintain genomic imprinting in comparison with in vivo-derived mouse embryos (Market-Velker et al., 2010). Khosla et al. showed that the presence of serum in culture medium reduces fetal weight and influences the regulation of multiple growth-related imprinted genes in mice (Khosla et al., 2001a). In humans, the effect of culture medium on gene expression is currently unknown.

The culture medium is the direct environment of the preimplantation embryo for several days during an IVF treatment. It seems that the embryo makes adaptations, likely by epigenetic modifications, during this short period of time, 
which will have long-lasting effects. In view of these results and the growing number of children conceived with ART, it is important that the effect of embryo culture medium is studied in the human more extensively, to select the optimal culture medium and to minimize short-term risks and perhaps even susceptibility to disease in later life.

\section{Conclusion and future prospects}

The effect of culture medium during the first few days after fertilization on human prenatal growth and birth weight persists during the first 2 years of postnatal life. This suggests that the human embryo is sensitive to its very early environment and that the culture medium used in IVF may have lasting consequences. This warrants further monitoring of the long-term growth, development and health of IVF children.

\section{Funding}

W.V. was funded with an unrestricted research grant from the Stichting Fertility Foundation. 



\section{CHAPTER 4}

\section{Age of G-1 PLUS v5 embryo culture medium is inversely associated with birth weight of the newborn}

Sander H.M. Kleijkers, Aafke P.A. van Montfoort, Luc J.M. Smits, Edith Coonen, Josien G. Derhaag, Johannes L.H. Evers, John C.M. Dumoulin

Human Reproduction 2015;30(6):1352-1357 


\section{Abstract}

Study question: Does age of G-1 PLUS v5 embryo culture medium affect IVF outcome?

Summary answer: Birth weight of singletons born after IVF showed an inverse association with age of the embryo culture medium, while no association was found between age of culture medium and fertilization rate, embryonic development or ongoing pregnancy.

What is known already: It has been reported that IVF culture media can deteriorate during storage, which suggests that the capacity of culture media to support optimal embryo development decreases over time. Some animal studies showed an effect of storage time on embryo development, in contrast to other studies, while the effect of aging culture medium on IVF outcome in humans is unknown.

Study design, size, duration: We used data on outcome of 1832 IVF/ICSI cycles with fresh embryo transfer, performed in the period 2008-2012 to evaluate the association of fertilization rate, embryonic development, ongoing pregnancy and birth weight of singletons with age of the culture medium (Vitrolife AB G-1 PLUS v5).

Participants/materials, setting, methods: Age of the culture medium was calculated by subtracting the production date from the date of ovum retrieval. Data analysis included linear regression and logistic regression on continuous and categorical outcomes, respectively.

Main results and the role of chance: Age of the culture medium was not associated with fertilization rate $(P=0.543)$, early cleavage rate $(P=0.155)$, percentage of embryos containing four or more cells on Day $2(P=0.401)$, percentage of embryos containing eight or more cells on Day $3(P=0.175)$, percentage of embryos with multinucleated blastomeres $(P=0.527)$, or ongoing pregnancy $(P=0.729)$. However, birth weight of the newborn was inversely associated with age of the medium ( $\beta=-3.6 \mathrm{~g}, \mathrm{SE}: 1.5 \mathrm{~g}, P=0.021)$, after controlling for possible confounders (day of embryo transfer, number of transferred embryos, child's gender, gestational age at birth, parity, pregnancy complications, maternal smoking, height and weight, and paternal height and weight) and the association was not biased by year of treatment, time since first opening of the bottle or batch variations. This indicates a difference of 234 grams in birth weight of newborns for media with an age difference of 65 days. 
Limitations, reasons for caution: The results from this study may be specific for the G-1 PLUS v5 culture medium and extrapolation of the results to other media should be done with caution because of the differences in composition and shelf life.

Wider implications of the findings: Age of G-1 PLUS v5 medium used to culture human embryos affects birth weight of the respective newborn. This could imply that the preimplantation embryo adapts to its in vitro environment with lasting in vivo consequences. Therefore, it is important that companies are transparent about the exact composition of their embryo culture media, which will allow IVF clinics to further investigate the effects of the media or media components on the health of IVF children.

Study funding/competing interest(s): No funding and no competing interests declared.

Trial registration number: Not applicable. 


\section{Introduction}

In the early days of IVF, embryo culture media consisted of simple salt solutions and were prepared by clinics in house (Lane and Gardner, 2007). Nowadays a wide range of commercial culture media for human preimplantation embryos is available, that have different compositions, varying from 10 up to 80 components, including salts, energy sources, proteins, antibiotics, amino acids, vitamins and growth factors (Moravek et al., 2012; Sunde and Balaban, 2013; Morbeck et al., 2014a).

It is important that culture media provide a proper environment to support in vitro embryo development. It has been demonstrated that culture media can deteriorate during storage (Stewart-Savage and Bavister, 1988; Weathersbee et al., 1995; Hossain et al., 2010). One well studied example of medium deterioration is the spontaneous breakdown of amino acids into ammonium (Gardner and Lane, 1993). This implies that the ability of culture media to support embryo development could theoretically decrease over time. Wales and Whittingham (1971) indeed reported that storage of culture medium at $5^{\circ} \mathrm{C}$ for 1 month resulted in decreased blastocyst formation in mice. In contrast, other studies found no decreased potential of mouse embryos to develop in vitro to the blastocyst stage in Ham's F-10 and M16 media that were stored at $4^{\circ} \mathrm{C}$ for several months, up to 14 months (Naz et al., 1986; Shioda and Tsunoda, 1986; De Silva, 1993).

Apart from these few studies, no papers have been published that addressed this issue. The aim of the present study was to investigate the effects of age of culture medium on embryo development, ongoing pregnancy and birth weight of the newborns in humans.

\section{Material and methods}

\section{Study design}

From January 2008 to December 2012, all 1832 IVF/ICSI cycles (except for cycles with donor oocytes or PGD) in which G-1 PLUS v5 (Vitrolife AB, Göteborg, Sweden) was used, were included to investigate the possible relationship between age of the medium and IVF outcome. Age of the medium was calculated for each cycle by subtracting the production date (which was cordially provided by Vitrolife $A B$ ) from the date of ovum retrieval. Registration of lot numbers (identification codes) and the day of first opening of a new bottle for our quality control system allowed us to determine the production date of the culture medium used and time since 
first opening of the bottle for each cycle. Before shipment, the embryo culture media were subjected to an internal quality control by the manufacturer. Embryo culture media were shipped in packages in which the temperature was maintained at $2-8^{\circ} \mathrm{C}$. Immediately upon arrival, the embryo culture media were stored in a refrigerator $\left(2-8^{\circ} \mathrm{C}\right)$ connected to a temperature real-time monitoring and alerting system XiltriX (IKS International, Torphins, UK). All media were used within 2 weeks after opening and before the expiry date. G-1 PLUS v5 is a readyto-use medium, which is supplemented with $5 \mathrm{mg} / \mathrm{ml}$ human serum albumin. Data on fertilization rate, embryonic development, ongoing pregnancy and, in cycles with a live born singleton after fresh embryo transfer, birth weight of the newborn, were collected. Fertilization rate refers to total number of fertilized (2PN) zygotes per total number of collected oocytes per cycle and early cleavage rate refers to total number of embryos with two cells approximately eight hours after fertilization per total number of embryos (originating from 2PN zygotes).

\section{IVF procedures}

Procedures in the IVF and ICSI treatments have been described (Kleijkers et al., 2014). Briefly, patients received long down-regulation with $\mathrm{GnRH}$ agonists and underwent ovarian stimulation with recombinant FSH. Ultrasound-guided oocyte retrieval was performed $36 \mathrm{~h}$ after hCG injection and progesterone was vaginally administered for luteal phase support. In case of a pregnancy, progesterone administration was continued for another 3 weeks.

The day before oocyte retrieval, dishes containing droplets of culture medium were prepared. In case of IVF, oocytes were inseminated in G-IVF PLUS v5 (Vitrolife $A B$ ) with approximately 50,000 progressively motile spermatozoa and the presence of pronuclei was checked at 18-20 h after insemination. Zygotes were subsequently transferred to G-1 PLUS v5 medium for individual culture in 5 $\mu \mathrm{l}$ droplets. In case of ICSI, oocytes were individually cultured in G-1 PLUS v5 medium immediately after injection. Culture droplets were covered by mineral oil (Irvine Scientific, Santa Ana, USA). Culture was performed in a $5 \% \mathrm{O}_{2}, 6 \% \mathrm{CO}_{2}$ and $89 \% \mathrm{~N}_{2}$ environment as instructed by the manufacturer. Embryo transfer was performed on Day 2 or, for reasons of convenience, on Day 3 after oocyte retrieval. Our standard transfer policy consisted of single embryo transfer when female age was $<38$ years and at least one good quality embryo was available. In all other cases, two embryos were transferred. Embryonic development was recorded daily and embryos that had reached the 4-cell stage on Day 2, or the 8cell stage on Day 3 , in combination with having the best morphological grade (regular, even sized blastomeres with $<20 \%$ fragmentation) and absence of multinucleated blastomeres (MNBs), were classified as good quality embryos. 


\section{Statistical analysis}

Linear regression analysis was used to study the association between age of the medium and fertilization rate, early cleavage rate, percentage of embryos with four or more cells on Day 2, percentage of embryos with eight or more cells on Day 3, percentage of embryos with MNBs, and birth weight. Logistic regression was used to investigate the association between age of the medium and ongoing pregnancy, with the number of embryos transferred (1 or 2) and the day of transfer ( 2 or 3 ) as covariates. As it is known that birth weight is affected by many factors, such as child's gender and maternal weight, the association between age of the medium and birth weight was further analyzed by the use of multiple linear regression, while controlling for the following covariates: day of transfer (Day 2 or Day 3), number of transferred embryos (1 or 2), child's gender, gestational age at birth, parity, pregnancy complications (gestational diabetes, hypertension and pre-eclampsia), maternal smoking, parental height and weight. All analyses were conducted with SPSS Statistics 20 (IBM, Armonk, USA) and two-sided $P$-values of $<0.05$ were considered to reflect statistical significance.

\section{Results}

\section{Embryo development and ongoing pregnancy}

From all 1832 IVF/ICSI cycles that were included in this study, no mature oocytes were retrieved in 13 cycles and in 4 cycles oocytes were vitrified for fertility preservation in cancer patients. For the remaining 1815 cycles, the cycle characteristics are presented in Table 1. Regression analysis showed that age of G1 PLUS $v 5$ medium had no effect on the embryo development characteristics and ongoing pregnancy as no association was found between age of the culture medium and fertilization rate $(P=0.543)$, early cleavage rate $(P=0.155)$, percentage of embryos containing four or more cells on Day $2(P=0.401)$, percentage of embryos containing eight or more cells on Day $3(P=0.175)$, percentage of embryos with multinucleated blastomeres $(P=0.527)$, and ongoing pregnancy $(P=0.709)$ (Table 2$)$. At time of arrival at the laboratory, age of the medium ranged from 35 to 113 days (mean: 79 days). All media were used after 291 days from arrival, but before the expiry date specified by the manufacturer, which corresponds to an age of the medium at ovum retrieval ranging from 82 to 147 days. 
Table 1. Cycle characteristics in a study of the effect of age of G-1 PLUS v5 embryo culture medium on IVF outcome in women.

\begin{tabular}{|c|c|}
\hline & Cycles $(n=1815)$ \\
\hline \multicolumn{2}{|l|}{ Primary indication for IVF/ICSI } \\
\hline Tubal & $208(11.5)$ \\
\hline Male subfertility & $1100(60.6)$ \\
\hline Unexplained & $322(17.7)$ \\
\hline Other & $185(10.2)$ \\
\hline \multicolumn{2}{|l|}{ Method of fertilization } \\
\hline IVF & $580(32.0)$ \\
\hline ICSI & $1235(68.0)$ \\
\hline Fertilization rate (\%) & $63.6 \pm 24.6$ \\
\hline Early cleavage rate (\%) & $28.7 \pm 31.9$ \\
\hline Embryos of $\geq 4$ cells on day $2(\%)$ & $68.8 \pm 30.7$ \\
\hline Good quality embryos on day 2 (\%) & $28.4 \pm 29.9$ \\
\hline Embryos of $\geq 8$ cells on day $3(\%)$ & $46.9 \pm 34.5$ \\
\hline Good quality embryos on day 3 (\%) & $25.6 \pm 30.3$ \\
\hline Embryos with MNBs (\%) & $18.5 \pm 23.3$ \\
\hline Single ET & $945(52.1)$ \\
\hline Double ET & $764(42.1)$ \\
\hline No ET & $106(5.8)$ \\
\hline \multicolumn{2}{|l|}{ Day of ET } \\
\hline Day 2 & $1076(59.3)$ \\
\hline Day 3 & $633(34.9)$ \\
\hline No ET & $106(5.8)$ \\
\hline Ongoing pregnancies after fresh ET & $449(24.7)$ \\
\hline
\end{tabular}

Data presented as mean \pm SD or number (\%).

MNB, multinucleated blastomeres; ET, embryo transfer.

Table 2. Regression analysis of age of G-1 PLUS v5 medium and several IVF outcomes in women.

\begin{tabular}{llll}
\hline Outcome variable & $\beta$ & SE & P-value \\
\hline Fertilization rate (\%) & 0.024 & 0.040 & 0.543 \\
Early cleavage rate (\%) & -0.079 & 0.056 & 0.155 \\
\% embryos of $\geq 4$ cells on day 2 & -0.043 & 0.051 & 0.401 \\
\% embryos of $\geq 8$ cells on day 3 & 0.082 & 0.060 & 0.175 \\
\% embryos with MNBs & 0.024 & 0.039 & 0.527 \\
Ongoing pregnancy (yes versus no) & -0.001 & 0.004 & 0.709 \\
\hline
\end{tabular}

$\beta$, regression coefficient. 


\section{Birth weight of the newborns}

From all live born singletons resulting from fresh embryo transfer ( $N=396)$, we were able to collect birth weight data of 372 (93.9\%) children. Parental and pregnancy characteristics for the corresponding cycles are presented in Table 3. A negative association ( $\beta=-4.2 \mathrm{~g}, \mathrm{SE}: 2.0 \mathrm{~g}, P=0.037$ ) was found with linear regression analysis between birth weight and age (in days) of the medium used during the IVF treatment (Figure 1). Multiple linear regression analysis showed that birth weight was still associated with age of the medium after controlling for possible confounders (height and weight of the parents, smoking habits of the mother, parity, pregnancy complications, gestational age at birth, gender of the child, number of transferred embryos and day of embryo transfer) ( $\beta=-3.6 \mathrm{~g}$, SE: $1.5 \mathrm{~g}, P=0.021$ ) (Table 4). This indicates that birth weight of a singleton newborn drops by 3.6 grams for every day the culture medium ages. This corresponds to a difference of 234 grams in birth weight for media with an age difference of 65 days (= the age range of the culture medium at the day of ovum retrieval in our center during the study period; 147 minus 82 days, see above).

Table 3. Parental and pregnancy characteristics for cycles with a live birth after fresh embryo transfer.

\begin{tabular}{lc}
\hline & Pregnancies $(\mathrm{n}=372)$ \\
\hline Maternal age (years) & $32.9 \pm 3.9$ \\
Maternal height $(\mathrm{cm})$ & $168 \pm 7$ \\
Maternal weight $(\mathrm{kg})$ & $69.3 \pm 10.7$ \\
Maternal smoking ( $\geq 10$ cigarettes/day) & $11(3.0)$ \\
Paternal age (years) & $36.6 \pm 5.8$ \\
Paternal height (cm) & $182 \pm 7$ \\
Paternal weight (kg) & $86.2 \pm 13.3$ \\
Primiparous & $256(68.8)$ \\
Singleton born from twin pregnancy & $4(1.1)$ \\
Pregnancy complications & $39(10.5)$ \\
Gestational age at birth (weeks) & $39.3 \pm 2.0$ \\
Preterm birth (<37 weeks) & $28(7.5)$ \\
Female newborns & $182(48.9)$ \\
\hline
\end{tabular}

Data presented as mean \pm SD or number (\%).

Alternative explanations for the association between age of the medium and birth weight, i.e. year of treatment, time since first opening of the bottle and batch variations, were investigated. Inclusion of the year of treatment or time since first opening of the bottle as variables into the multiple regression analysis resulted in similar outcomes for age of medium $(\beta=-3.8, \mathrm{SE}=1.5, P=0.010$ and $\beta=-3.6, \mathrm{SE}=$ $1.5, P=0.020$, respectively), while the added variables were not significantly associated with birth weight. Inclusion of the batches, which could have differed 
due to subtle variations in protein supplement (Zhu et al., 2014a), into the multiple regression analysis was not possible due to the number of batches ( $n=$ 56). However, there were no differences in birth weight between the different batches $(P=0.191)$ when analyzed by one-way analysis of variance. These results show that the association between age of the medium and birth weight was not biased by year of treatment, time since first opening of the bottle or batch variations.

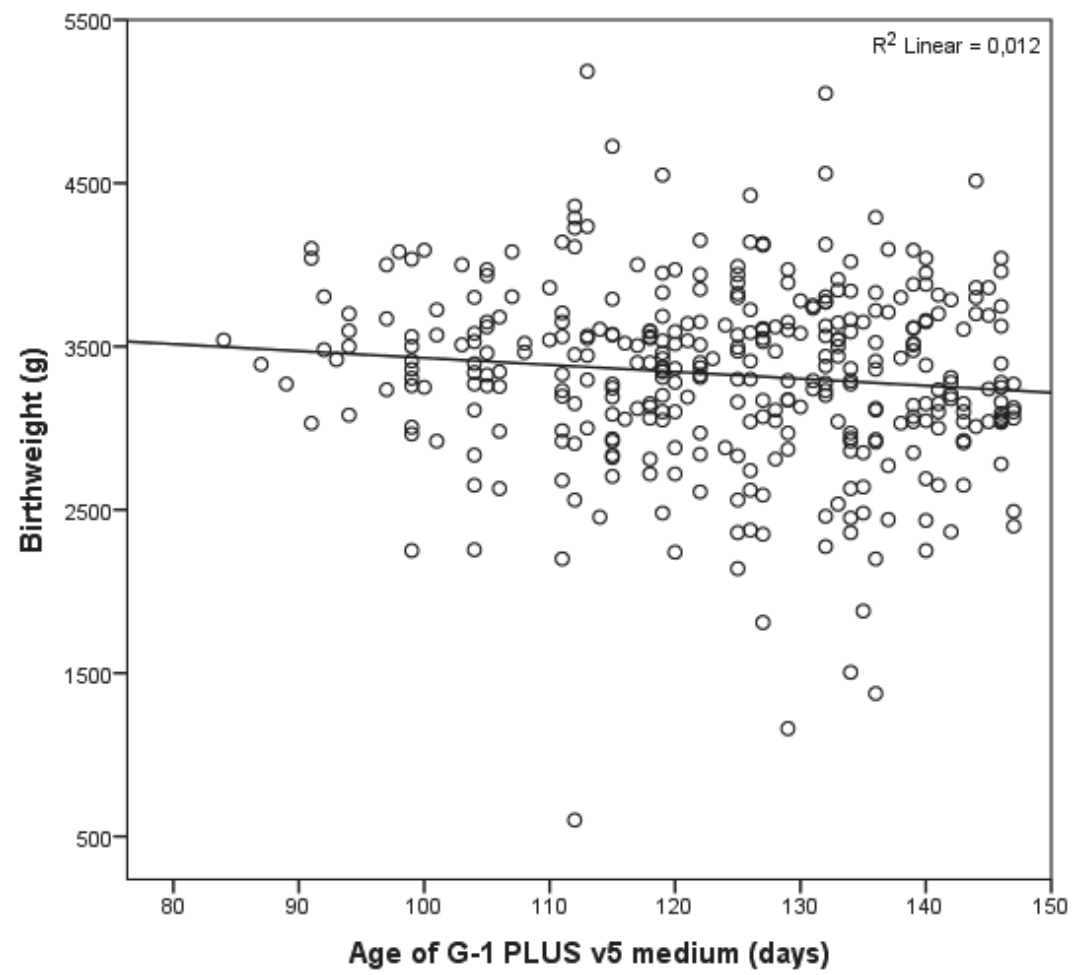

Figure 1. Correlation between birth weight and age of G-1 PLUS v5 medium. Birth weight of the live born singletons after fresh embryo transfer is negatively affected by age of the medium used during the IVF treatment $(\beta=-4.245 \mathrm{~g}$, SE: $2.023 \mathrm{~g}, \mathrm{P}=0.037)$. 
Table 4. Results of multiple regression analysis of birth weight among live born singletons after transfer of fresh embryos.

\begin{tabular}{lccc}
\hline Outcome variable & \multicolumn{1}{c}{ SE } & P-value \\
\hline Age of medium (per day) & -3.6 & 1.5 & 0.021 \\
Day of transfer (3 versus 2) & -3.7 & 47.9 & 0.938 \\
Number of transferred embryos (2 versus 1) & -83.7 & 49.0 & 0.089 \\
Child's gender (male versus female) & 110.0 & 45.1 & 0.015 \\
Gestational age at birth (per week) & 177.9 & 12.0 & $<0.001$ \\
Parity (primiparous versus multiparous) & 129.1 & 49.9 & 0.010 \\
Pregnancy complications (yes versus no) & -2.8 & 72.3 & 0.969 \\
Maternal smoking (<10 versus $\geq 10$ cig/day) & -12.1 & 8.1 & 0.138 \\
Maternal height (per cm) & 13.9 & 3.8 & $<0.001$ \\
Maternal weight (per kg) & 6.9 & 2.4 & 0.005 \\
Paternal height (per cm) & 9.7 & 3.7 & 0.009 \\
Paternal weight (per kg) & -2.2 & 2.0 & 0.283 \\
\hline
\end{tabular}

$\beta$, regression coefficient.

\section{Discussion}

This study shows that the age of G-1 PLUS v5 culture medium is inversely associated with birth weight of human newborns. No association with embryo development and ongoing pregnancy was found.

Only few animal studies have been published on the effect of stored culture medium on the culture of gametes and embryos. Modified Tyrode's solutions showed deterioration after 2-4 weeks storage at $5^{\circ} \mathrm{C}$, as monitored by a sperm motility bioassay (Stewart-Savage and Bavister, 1988). Wales and Whittingham (1971) reported a decreased blastocyst formation of 2-cell mouse embryos cultured in sterile medium that was stored at $5^{\circ} \mathrm{C}$ for 1 month. Contrary to this, storage of M16 medium for 90 days in the refrigerator showed no effect on the in vitro development of mouse embryos to the blastocyst stage and the implantation rates after transfer of blastocysts to recipients (Shioda and Tsunoda, 1986). Also, storage of Ham's F-10 medium at $4^{\circ} \mathrm{C}$ for 1 to 6 months (De Silva, 1993) or 150 to 425 days (Naz et al., 1986) did not affect blastocyst formation in mice. In line with these findings, we found that the age of G-1 PLUS v5 medium was not associated with human embryo development and ongoing pregnancy. However, an association between birth weight of the newborns and the age of the medium was found. As far as we know, no other studies have reported on the effect of aging culture medium on the birth weight of the offspring. 
Birth weight is affected by many factors, i.e. maternal smoking habits, parental height and weight, parity, child's gender and gestational age at birth (Kramer, 1987). Furthermore, IVF related factors, such as cryopreservation of embryos (Pinborg et al., 2010) and number of transferred embryos (De Sutter et al., 2006), have been associated with birth weight of the newborns. Moreover, prolonged in vitro culture until the blastocyst stage resulted in heavier babies at birth compared with in vitro culture until the cleavage stage (Makinen et al., 2013; Zhu et al., 2014b). Previous studies from our group, as well as work from others, showed that the use of different culture media resulted in differences in birth weight (Dumoulin et al., 2010; Nelissen et al., 2012; Eskild et al., 2013; Hassani et al., 2013; Zhu et al., 2014a). These studies indicate that birth weight of the newborn is affected by the environment it has encountered as an embryo during the preimplantation stage. In the present study, it is not unlikely that aging of the culture medium resulted in a changed embryonic environment. This is in line with findings that gametes and preimplantation embryos adapt to their environment with long-term health consequences (Khosla et al., 2001b; Steegers-Theunissen et al., 2013). For example, culturing mouse embryos in medium supplemented with serum affected the regulation of multiple growth-related imprinted genes in the fetuses, which resulted in aberrant fetal growth and development (Khosla et al., 2001a). The exposure of preimplantation mouse embryos to palmitic acid caused altered insulin-like growth factor 1 receptor expression, metabolism and growth, which resulted in smaller pups at delivery (Jungheim et al., 2011). In the present study, it is possible that the gene expression in embryos is compromised by the aged culture medium, which results in aberrant fetal growth.

In the last four decades, only a small number of studies reported on the deterioration of stored culture media. Wales and Whittingham (1971) reported on the formation of parapyruvate from the spontaneous decarboxylation of pyruvate in sterile medium during storage at $5^{\circ} \mathrm{C}$ over 3 months. In a study by Weathersbee et al. (1995) which evaluated the use of a basic medium, which had to be supplemented with a freeze-dried portion containing L-glutamine and sodium pyruvate, it was found that certain amino acids and vitamins are unstable in the liquid basic medium after 18 months of storage at $2-8^{\circ} \mathrm{C}$. One well studied example of medium deterioration is the accumulation of ammonium in the culture medium during incubation due to the spontaneous breakdown of amino acids (Gardner and Lane, 1993). Several studies showed that ammonium has a negative effect on fetal development in animals (Lane and Gardner, 1994; Lane and Gardner, 2003; Sinawat et al., 2003; Zander et al., 2006). However, most of these studies used relatively high levels of exogenous ammonium to study the effects. Yet Lane and Gardner (2003) reported that with low levels of ammonium it was possible to generate morphologically normal-appearing blastocysts that are 
compromised in metabolism, expression of the imprinted growth regulator gene $\mathrm{H} 19$ and future fetal development.

In an unpublished study, we found relatively low levels of ammonium accumulation during storage for 6 weeks at $2-8^{\circ} \mathrm{C}$ in 30 commercially available albumin solutions and embryo culture media, including G-1 PLUS v5. This suggests that embryos in the present study faced a less than optimal environment when cultured in older medium. However, we did not measure deterioration of other components in the media, such as amino acids and vitamins, in the present study, that could also potentially affect birth weight. Furthermore, the stability of a medium has been shown to depend on its components, which in addition determines its shelf life (Gardner and Lane, 2004). It would be interesting to know how the manufacturer determines the recommended shelf life of G-1 PLUS v5 and which components in particular influence this decision.

The results from this study may be specific for the G-1 PLUS v 5 culture medium and extrapolation of the results to other media should be done with caution because of the differences in compositions and shelf lives.

Nonetheless, this study is another indication that the early environment affects the human embryo, with long-term consequences. It has been shown that preimplantation embryos can adapt to their environment with effects that extend to fetal and post-natal development and even may affect susceptibility to disease in later life (Duranthon et al., 2008; Watkins and Fleming, 2009).

\section{Conclusion and future prospects}

Age of G-1 PLUS v5 medium affects birth weight of the human newborn. This may imply adaptations of the preimplantation embryo to its environment with lasting consequences. Therefore, it is important that companies are transparent about the exact composition of their embryo culture media, which will allow IVF clinics to further investigate the effects of the media or media components on the health of IVF children. 


\section{CHAPTER 5}

Ammonium accumulation in commercially available embryo culture media and protein supplements during storage at $2-8^{\circ} \mathrm{C}$ and during incubation at $37^{\circ} \mathrm{C}$

Sander H.M. Kleijkers, Aafke P.A. van Montfoort, Otto Bekers, Edith Coonen, Josien G. Derhaag, Johannes L.H. Evers, John C.M. Dumoulin

Human Reproduction 2016;31(6):1192-1199 


\section{Abstract}

Study question: Does ammonium accumulate in commercially available culture media and protein supplements used for in vitro development of human preimplantation embryos during storage and incubation?

Summary answer: Ammonium accumulates in ready-to-use in vitro fertilization (IVF) culture media during storage at $2-8^{\circ} \mathrm{C}$ and in ready-to-use IVF culture media and protein supplements during incubation at $37^{\circ} \mathrm{C}$.

What is known already: Both animal and human studies have shown that the presence of ammonium in culture medium has detrimental effects on embryonic development and pregnancy rate. It is, therefore, important to assess the amount of ammonium accumulation in ready-to-use IVF culture media under conditions that are common in daily practice.

Study design, size, duration: Ammonium accumulation was investigated in 15 ready-to-use media, 11 protein-free media and 8 protein supplements.

Participants/materials, setting, methods: Ammonium was measured by the use of an enzymatic method with glutamate dehydrogenase. To simulate the storage and incubation conditions during IVF treatments, ammonium concentrations were measured at different time-points during storage at $2-8^{\circ} \mathrm{C}$ for 6 weeks and during incubation at $37^{\circ} \mathrm{C}$ for 4 days.

Main results and the role of chance: All ready-to-use, i.e. protein supplemented, culture media showed ammonium accumulation during storage for 6 weeks (ranging from 9.2 to $99.8 \mu \mathrm{M}$ ) and during incubation for 4 days (ranging from 8.4 to $138.6 \mu \mathrm{M}$ ), resulting in levels that might affect embryo development. The protein supplements also showed ammonium accumulation, while the culture media without protein supplementation did not. The main sources of ammonium buildup in ready-to-use culture media were unstable glutamine and the protein supplements. No additional ammonium buildup was found during incubation when using an oil overlay or with the presence of an embryo in the culture droplet.

Limitations, reasons for caution: In addition to the unstable glutamine and the protein supplements, other free amino acids might contribute to the ammonium buildup. We did not investigate the deterioration of other components in the media.

Wider implications of the findings: Breakdown of components into ammonium is more pronounced during incubation at $37^{\circ} \mathrm{C}$, however, it is not negligible during 
storage at $2-8^{\circ} \mathrm{C}$. This results in increasing ammonium levels in culture media over time that may affect embryo development. Therefore, it is important that the use of free L-glutamine in human embryo culture media is stopped and that the use of protein supplements is thoroughly evaluated.

Study funding/competing interest(s): No funding or no competing interests declared.

Trial registration number: Not applicable. 


\section{Introduction}

Commercially available embryo culture media are routinely used to culture human preimplantation embryos for 2-6 days during an in vitro fertilization (IVF) treatment. Culture media are considered to be an important factor in IVF, as they can affect live birth rate, pregnancy rate, implantation rate, fertilization rate and the number of good quality embryos (Mantikou et al., 2013b). Amino acids have been added to most embryo culture media, as it has been shown that they have a beneficial effect on preimplantation embryo development in several species (Bavister, 1995; Gardner and Lane, 1997; Biggers and Summers, 2008). However, it is known that amino acids, especially free L-glutamine, spontaneously break down into ammonium during incubation at $37^{\circ} \mathrm{C}$ (Gardner and Lane, 1993).

Studies with animal models have shown that the addition of exogenous ammonium to embryo culture media has detrimental effects on embryonic development, pregnancy rates and fetal development (Lane and Gardner, 1994; Hammon et al., 2000; Lane and Gardner, 2003; Sinawat et al., 2003; Zander et al., 2006; Gardner et al., 2013). Especially during the cleavage stage, embryos are vulnerable to ammonium in culture media (Zander et al., 2006; Gardner et al., 2013). In human studies, it was found that elevated ammonium levels in culture media negatively affect blastocyst formation (Virant-Klun et al., 2006; Hashimoto et al., 2008) and result in the altered expression of 390 genes involved in metabolism, cell growth and/or maintenance, transcription, cell communication, transport, development and regulation of transcription (Gardner et al., 2013).

To reduce the ammonium accumulation in embryo culture media, the unstable Lglutamine has been replaced with a dipeptide form, e.g. alanyl-glutamine or glycyl-glutamine, in most embryo culture media. However, in these media, a slight ammonium accumulation has been observed (Gilbert et al., 2012; Li et al., 2013). Apart from amino acids, other factors, such as the protein supplementation, might contribute to the ammonium accumulation during culture (Meintjes, 2012). The aim of the present study was to assess ammonium accumulation in currently commercially available culture media with and without protein supplementation and in separate protein supplements used for in vitro development of human preimplantation embryos. 


\section{Materials and methods}

\section{Culture media}

Ammonium buildup was investigated in protein supplements and both ready-touse (protein supplemented) and protein-free culture media from different manufacturers.

The ready-to-use culture media were Cook Sydney IVF Fertilization Medium (KSIFM-20), Cook Sydney IVF Cleavage Medium (K-SICM-20), Cook Sydney IVF Blastocyst Medium (K-SIBM-20), Sage Quinn's Advantage Protein Plus Fertilization Medium (ART-1520), Sage Quinn's Advantage Protein Plus Cleavage Medium (ART-1526), Sage Quinn's Advantage Protein Plus Blastocyst Medium (ART-1529), Irvine Complete Early Cleavage Medium with DSS (90142), Irvine Complete MultiBlast Medium with DSS (90143), Irvine Continuous Single Culture Complete Medium (90165), Global Total for Fertilization (LGTF-050), Global Total Medium (LGGT-030), Origio ISM1 Culture Medium (1150), Vitrolife G-IVF PLUS v5 (10136), Vitrolife G-1 PLUS v5 (10128) and Vitrolife G-2 PLUS v5 (10132) (Supplementary data, Table S1; available online).

The protein-free culture media were Sage Quinn's Advantage Fertilization Medium (ART-1020), Sage Quinn's Advantage Cleavage Medium (ART-1026), Sage Quinn's Advantage Blastocyst Medium (ART-1029), Irvine Early Cleavage Medium (90138), Irvine MultiBlast Medium (90139), Global for Fertilization (LGGF-020), Global Medium (LGGG-020), Lonza Human Tubal Fluid (HTF) Medium (BE02-036F), Vitrolife G-IVF (10135), Vitrolife G-1 (10127) and Vitrolife G-2 (10131) (Supplementary data, Table S2; available online).

The protein supplements were Sage Human Serum Albumin (HSA) (ART-3001), Sage Serum Protein Substitute (SPS; ART-3011), Irvine HSA (9988), Irvine Serum Substitute Supplement (SSS; 99193), Global HSA (GHSA-125), Sanquin Albuman (21-136), Vitrolife HSA (10064) and Vitrolife G-MM (10038) (Supplementary data, Table S3; available online). Furthermore, two freshly made glutamine solutions were tested; $1 \mathrm{mM}$ of L-Glutamine (Sigma-Aldrich, St. Louis, USA; G8540-25G) and $1 \mathrm{mM}$ of Glycyl-Glutamine (Sigma-Aldrich, St. Louis, USA; G5149-1G) in HTF medium (Lonza, Verviers, Belgium; BE02-036F). Glutamine is frequently used at a concentration of 0.5-1 mM in embryo culture (Gardner, 2007) and HTF is a medium devoid of amino acids and protein.

Immediately upon arrival, ordered culture media and protein supplements were stored in a refrigerator $\left(2-8^{\circ} \mathrm{C}\right)$ connected to a temperature real-time monitoring and alerting system XiltriX (IKS International, Torphins, UK). 


\section{Ammonium measurements}

To simulate the storage and incubation conditions during IVF treatments, ammonium concentrations were measured at different time-points during storage at $2-8^{\circ} \mathrm{C}$ and during incubation at $37^{\circ} \mathrm{C}\left(90 \%\right.$ relative humidity, $\left.6 \% \mathrm{CO}_{2}, 5 \% \mathrm{O}_{2}\right)$. Incubation was performed without gametes or embryos for 2 days in case of fertilization medium and for 4 days in case of the cleavage and blastocyst medium; i.e. the number of days the medium would be in the incubator during a normal IVF treatment, taking into account that the medium is placed in the incubator a day before use to allow equilibration. At the time of ammonium analysis, samples were collected and transported on ice to the Central Diagnostic Laboratory of our hospital, where the samples were measured immediately.

Ammonium was measured by an enzymatic method with glutamate dehydrogenase (GLDH). This method is based on the chemical reaction in which glutamate and $\mathrm{NADP}^{+}$are formed by the reductive amination of 2-oxoglutarate with $\mathrm{NH}_{4}^{+}$and $\mathrm{NADPH}$, which is catalyzed by GLDH. The ammonium concentration is directly proportional to the concentration of the formed NADP ${ }^{+}$, which is determined by measuring the decrease in absorbance by using the Cobas 6000 Analyzer Series (Roche Diagnostics, Basel, Switzerland). As the Cobas 6000 Analyzer has been calibrated for analysis of plasma, the method has been validated for the analysis of embryo culture media. A serum control sample (218.2 $\left.\mu \mathrm{M} \mathrm{NH}^{+}\right)$and an embryo culture medium sample $\left(46.3 \mu \mathrm{M} \mathrm{NH} 4^{+}\right)$have been used to create a dilution series $(1: 1,1: 3,1: 6$ and $1: 9)$. Measured concentrations were similar to the theoretical concentrations, with a deviation ranging from $-0.2 \%$ to $3.6 \%$ (average $1.3 \%$ ). This indicates that there is no matrix effect. Precision was determined at two different concentrations (99.4 $\mathrm{MM} \mathrm{NH}_{4}{ }^{+}$and $203.1 \mathrm{\mu M} \mathrm{NH}_{4}{ }^{+}$), $\mathrm{CV}$ (within-run) values were 2.1 and $3.1 \%$, CV (between-day) values were 1.3 and $1.7 \%$, and the total imprecision was 3.7 and $2.1 \%$, respectively. The minimal detection threshold was $10 \mu \mathrm{M}$.

All ammonium concentrations are given as mean values of duplicate measurements and for all media two or three different batches were used to investigate the average ammonium accumulation, except for Sage SPS, which was discontinued in the Netherlands during this study by the manufacturer due to the absence of Conformité Européenne (CE) marking. For the construction of the ammonium buildup curves, the measurement at the first time-point of each medium or solution was set to zero and all measurements at subsequent time-points were related to this initial measurement. This was done to allow a reliable comparison in ammonium buildup between the different culture media, as age of the medium (from the time point of manufacture) upon arrival at our department was unknown for some of the media. First measurements started within 2 weeks of the arrival of the medium at the laboratory and all measurements were performed before the expiry date. 
Statistical analysis

Differences in ammonium buildup between the two glutamine solutions and between culture media were tested by use of Student's $t$-test and by use of oneway analysis of variance followed by Scheffe's post hoc multiple comparisons test, respectively.

\section{Results}

\section{Ammonium production in ready-to-use culture media}

The ammonium buildup during storage in the refrigerator at $2-8^{\circ} \mathrm{C}$ in several readyto-use culture media, which are already supplemented with protein, are presented in Figure 1. The average accumulation during storage was lowest in fertilization medium from Global $(10.0 \pm 1.2 \mu \mathrm{M})$, Irvine $(14.0 \pm 2.1 \mu \mathrm{M})$, Vitrolife $(15.8 \pm 2.9$ $\mu \mathrm{M})$, Irvine CSC $(22.5 \pm 0.5 \mu \mathrm{M})$ and Cook $(24.8 \pm 3.7 \mu \mathrm{M})$. The ammonium buildup was significantly higher in the Sage medium $(38.8 \pm 1.0 \mu \mathrm{M})$ when compared with the Global, Irvine, Vitrolife or Irvine CSC medium, however, it was highest in the Origio ISM1 medium, which reached a level of $99.8 \pm 3.5 \mu \mathrm{M}$ in 6 weeks (Fig. 1A). In the cleavage media (Fig. 1B), the ammonium buildup during storage was not significantly different between Global $(9.2 \pm 0.7 \mu \mathrm{M})$, Irvine $(14.0 \pm 2.1 \mu \mathrm{M})$, Vitrolife $(20.5 \pm 2.8 \mu \mathrm{M})$, Irvine CSC $(22.5 \pm 0.5)$, Sage $(28.2 \pm 6.2 \mu \mathrm{M})$ and Cook $(28.8 \pm 1.1 \mu \mathrm{M})$, while the ammonium buildup was significantly higher in the Origio ISM1 medium $(99.8 \pm 3.5 \mu \mathrm{M})$. In the blastocyst media (Fig. 1C), the average ammonium buildup during storage was not significantly different between Global $(9.2 \pm 0.7 \mu \mathrm{M})$, Sage $(13.3 \pm 0.7 \mu \mathrm{M})$, Irvine $(22.0 \pm 3.1 \mu \mathrm{M})$, Cook $(22.1 \pm 3.1 \mu \mathrm{M})$, Irvine CSC $(22.5 \pm 0.5 \mu \mathrm{M})$ and Vitrolife $(24.2 \pm 4.8 \mu \mathrm{M})$.

The ammonium buildup in several ready-to-use culture media during incubation at $37^{\circ} \mathrm{C}$ are presented in Figure 2. The average accumulation during incubation was lowest in fertilization medium from Vitrolife $(8.4 \pm 1.6 \mu \mathrm{M})$, Irvine $(8.9 \pm 2.0 \mu \mathrm{M})$, Global $(11.2 \pm 0.6 \mu \mathrm{M})$, Cook $(13.5 \pm 0.9 \mu \mathrm{M})$ and Irvine CSC $(14.2 \pm 1.8)$. The ammonium buildup was higher in the Sage medium $(22.6 \pm 2.9 \mu \mathrm{M})$ when compared with the Vitrolife or Irvine medium, however, it was highest in the Origio ISM1 medium, which reached a level of $85.4 \pm 3.0 \mu \mathrm{M}$ in 2 days (Fig. 2A). In cleavage media (Fig. 2B), the ammonium buildup during incubation was not significantly different between the media from Sage $(10.6 \pm 1.0 \mu \mathrm{M})$, Irvine $(13.7 \pm$ $2.8 \mu \mathrm{M})$, Vitrolife $(15.3 \pm 2.0 \mu \mathrm{M})$ and Global $(17.8 \pm 0.9 \mu \mathrm{M})$. The ammonium buildup in Cook $(24.8 \pm 1.6 \mu \mathrm{M})$ was only significantly higher when compared with Sage, while Irvine CSC $(26.3 \pm 0.8 \mu \mathrm{M})$ was significantly higher when compared with the Sage, Irvine or Vitrolife medium and ammonium buildup was highest in the 
Origio ISM1 medium (138.6 $\pm 1.9 \mu \mathrm{M})$. In blastocyst media (Fig. 2C), the average ammonium buildup during incubation was significantly lower in Sage medium (6.6 $\pm 1.1 \mu \mathrm{M})$ when compared with Global $(17.8 \pm 0.9 \mu \mathrm{M})$, Irvine $(19.5 \pm 1.1 \mu \mathrm{M})$, Vitrolife $(23.4 \pm 2.4 \mu \mathrm{M})$, Cook $(25.8 \pm 3.6 \mu \mathrm{M})$ and Irvine CSC $(26.3 \pm 0.8 \mu \mathrm{M})$.

A

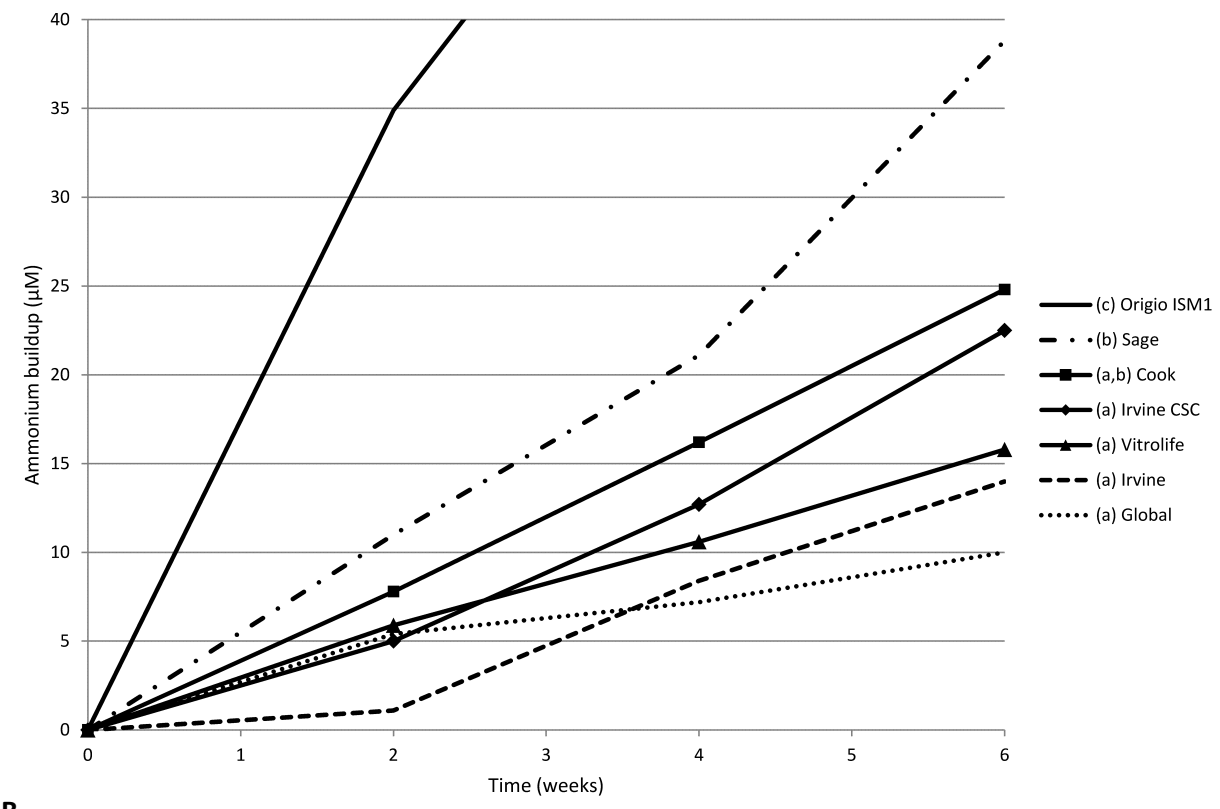

B

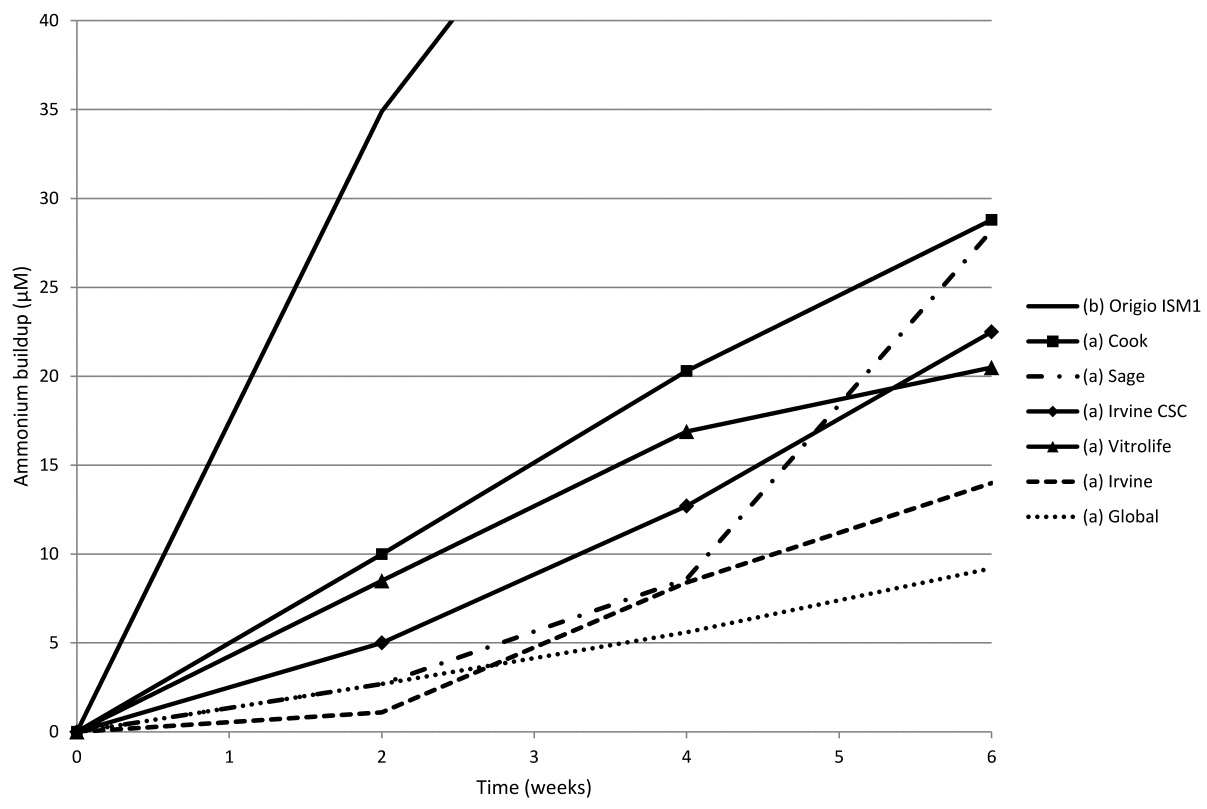


C

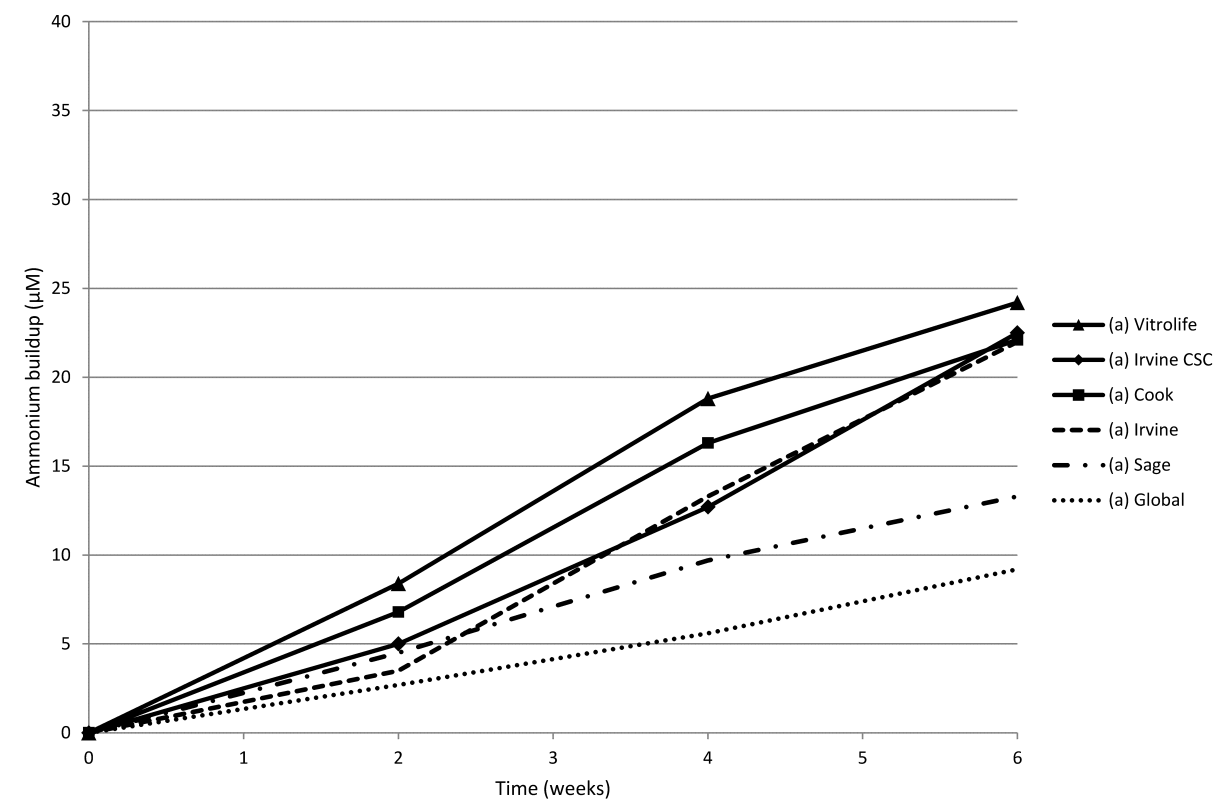

Figure 1. Average ammonium buildup in ready-to-use, protein supplemented, media during storage at $2-8^{\circ} \mathrm{C}$. The fertilization media (A) and cleavage media (B) from Cook, Global, Irvine, Origio, Sage and Vitrolife. The average ammonium buildup of the Origio ISM1 culture medium, which contains the mono-peptide L-glutamine, was $66.1 \mu \mathrm{M}$ in 4 weeks and $99.8 \mu \mathrm{M}$ in 6 weeks (not displayed in figures A and B). The blastocyst media (C) from Cook, Global, Irvine, Sage and Vitrolife. Different letters in the legend indicate significant difference $(P<$ 0.05 ) in ammonium buildup at 6 weeks. SEMs are given in the main text. 
Chapter 5

A

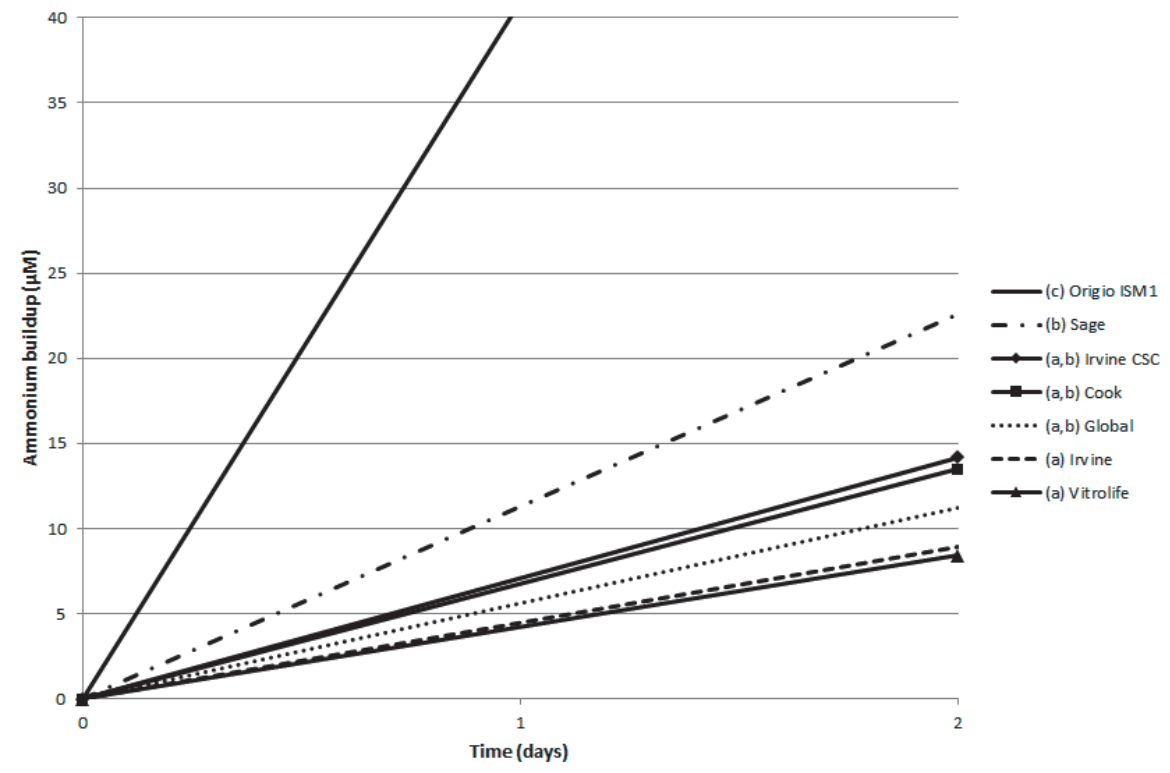

B

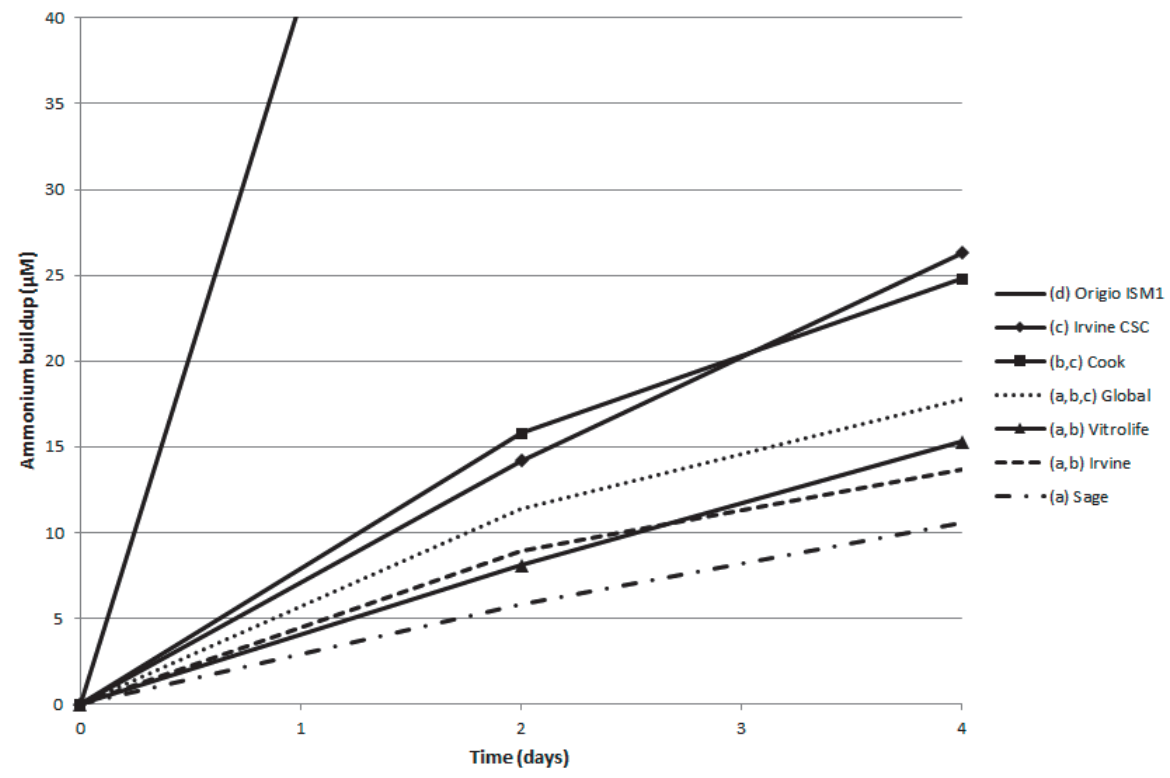

76 
C

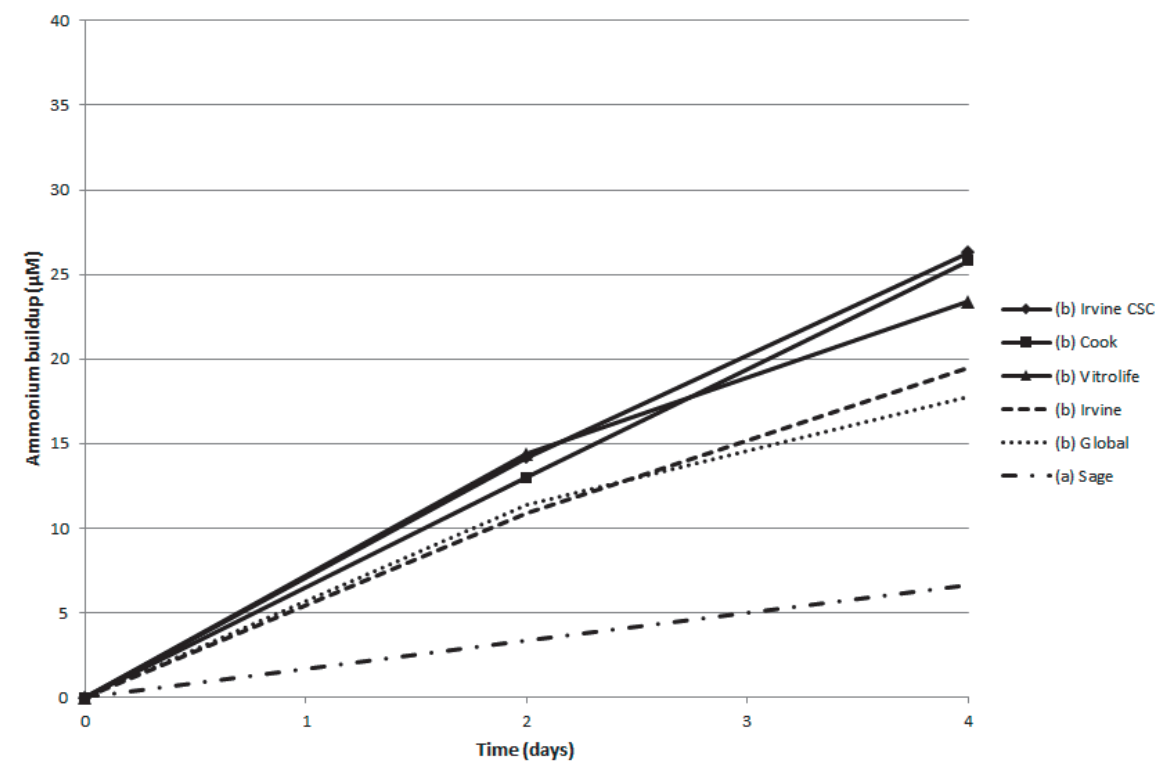

Figure 2. Average ammonium buildup in ready-to-use, protein supplemented, media during incubation at $37^{\circ} \mathrm{C}$. The fertilization media (A) and cleavage media (B) from Cook, Global, Irvine, Origio, Sage and Vitrolife. The average ammonium buildup of the Origio ISM1 culture medium, which contains the mono-peptide L-glutamine, was $85.4 \mu \mathrm{M}$ in 2 days and $138.6 \mu \mathrm{M}$ in 4 days (not displayed in figures $A$ and $B$ ). The blastocyst media (C) from Cook, Global, Irvine, Sage and Vitrolife. Different letters in the legend indicate significant difference $(P<0.05)$ in ammonium buildup at Day 2 (A) or at Day $4(B, C)$. SEMs are given in the main text.

\section{Ammonium production in glutamine solutions}

The Origio ISM1 medium was the only medium in this study that contained the relatively unstable free L-glutamine, which might be the cause of the high ammonium buildup. Two glutamine solutions were prepared, one with the unstable free L-glutamine and one with a stable dipeptide of glutamine, to verify the difference in ammonium buildup.

The average ammonium buildup during storage at $2-8^{\circ} \mathrm{C}$ and during incubation at $37^{\circ} \mathrm{C}$ in solutions with L-glutamine or with the dipeptide glycyl-glutamine are presented in Table 1. There was no detectable ammonium buildup during storage for 6 weeks and little buildup during incubation for 4 days in the glycyl-glutamine solution. There was significantly more ammonium production in the L-glutamine solution, both during storage for 6 weeks and incubation for 4 days (230.6 \pm 2.5 $\mu \mathrm{M}$ and $208.0 \pm 3.5 \mu \mathrm{M}$ respectively). 
Table 1. Average ammonium buildup $(\mu \mathrm{M})$ in solutions of L-glutamine (1 mM) and glycyl-glutamine (1 mM) during storage at $2-8^{\circ} \mathrm{C}$ and during incubation at $37^{\circ} \mathrm{C}$.

\begin{tabular}{lllll}
\hline Condition & Time-point & $\begin{array}{l}\text { L-glutamine } \\
\text { Ammonium buildup }(\mu \mathrm{M})\end{array}$ & $\begin{array}{l}\text { Glycyl-glutamine } \\
\text { Ammonium buildup }(\mu \mathrm{M})\end{array}$ & P-value \\
\hline Storage & Week 2 & $58.0 \pm 0.9$ & $0.0 \pm 0.0$ & $<0.001$ \\
Storage & Week 4 & $130.5 \pm 4.1$ & $0.0 \pm 0.0$ & 0.001 \\
Storage & Week 6 & $230.6 \pm 2.5$ & $0.0 \pm 0.0$ & $<0.001$ \\
Incubation & Day 2 & $103.6 \pm 3.4$ & $0.0 \pm 0.0$ & 0.001 \\
Incubation & Day 4 & $208.0 \pm 3.5$ & $7.5 \pm 1.3$ & $<0.001$ \\
\hline
\end{tabular}

Data presented as mean \pm SEM.

\section{Ammonium production in protein-free culture media and protein supplements}

To investigate the role of the protein supplements in the ammonium accumulation, ammonium buildup was first evaluated in protein-free culture media. Both during storage at $2-8^{\circ} \mathrm{C}$ for 6 weeks and incubation at $37^{\circ} \mathrm{C}$ for 4 days, there was no detectable ammonium buildup in these culture media (data not shown). This indicates that the protein supplement might be another source for the ammonium buildup. Next, the average ammonium buildup in protein supplements was measured (Figure 3). All protein supplements showed ammonium buildup during incubation for 4 days, ranging from $18.9 \mu \mathrm{M}$ in Vitrolife G-MM up to $144.7 \mu \mathrm{M}$ in Irvine SSS. No statistical comparisons between groups were performed, since the protein supplements were used undiluted and the concentrations of protein were not the same in all solutions.

\section{Ammonium production during embryo culture and with oil overlay}

In order to assess the effect of the presence of an embryo in the culture medium, we collected 26 pooled droplets ( $15 \mu \mathrm{l}$ ) of Vitrolife G-1 PLUS v5 incubated with an embryo (originating from 2PN zygotes) and 26 pooled droplets ( $15 \mu \mathrm{l}$ ) of Vitrolife G-1 PLUS v5 incubated without an embryo from the same culture dish, cultured in a $5 \% \mathrm{O}_{2}, 6 \% \mathrm{CO}_{2}$ and $89 \% \mathrm{~N}_{2}$ environment. From both pools, $200 \mu \mathrm{l}$ was taken to compare ammonium levels and no additional ammonium buildup was detected by the presence of an embryo in the medium droplet at Day 3 after ovum retrieval. Furthermore, the effect of a mineral oil overlay was evaluated by comparing the ammonium levels of Vitrolife G-1 PLUS v5 with and without a mineral oil overlay (Irvine Scientific, \# 9305), which resulted in similar ammonium buildup after 4 days of incubation at $37^{\circ} \mathrm{C}$. 


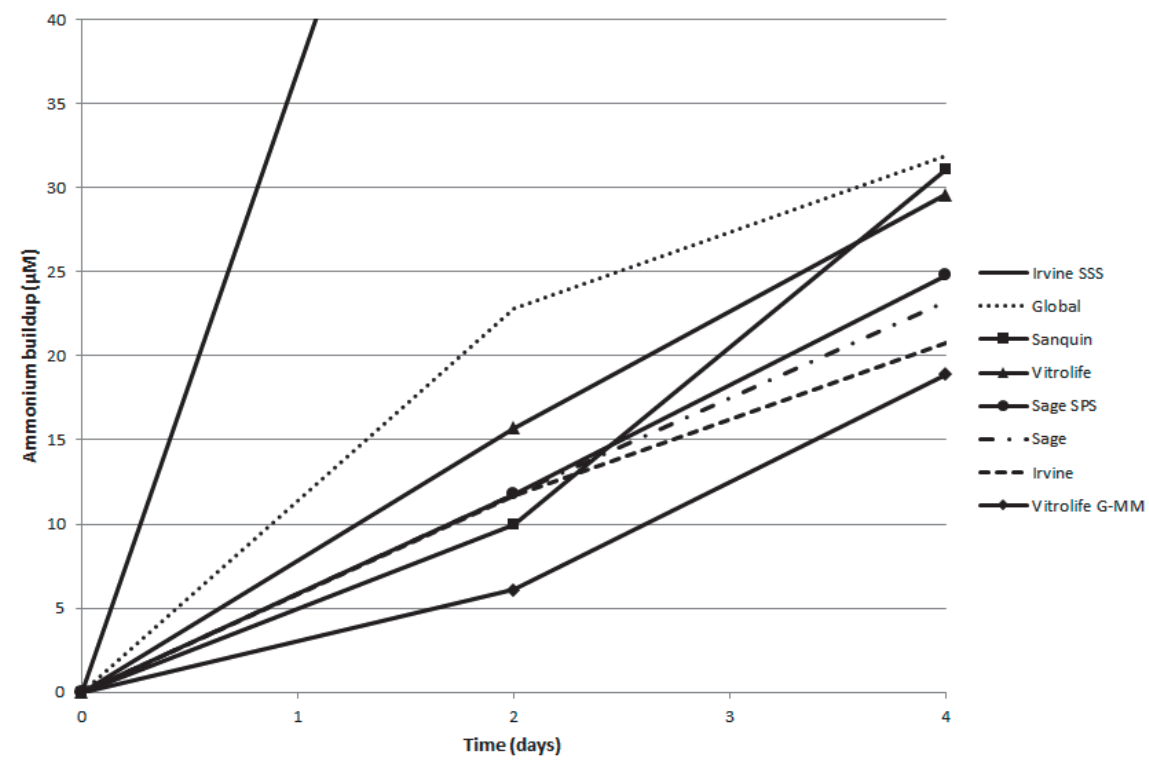

Figure 3. Average ammonium buildup in undiluted protein supplements from Global $(100 \mathrm{mg} / \mathrm{mL} H S A)$, Irvine (100 mg/mL HSA), Irvine SSS (50 mg/ mL HSA + $10 \mathrm{mg} / \mathrm{mL} \alpha$ - and $\beta$-globulins), Sage (100 mg/mL HSA), Sage SPS (44 mg/mL HSA $+6 \mathrm{mg} / \mathrm{mL} \alpha$ - and $\beta$-globulins), Sanquin (40 mg/mL HSA), Vitrolife $(100 \mathrm{mg} / \mathrm{mL} \mathrm{HSA})$ and Vitrolife G-MM $(50 \mathrm{mg} / \mathrm{mL}$ recombinant $\mathrm{HSA})$ during incubation at $37^{\circ} \mathrm{C}$. The average ammonium buildup of Irvine SSS was $73.9 \mu \mathrm{M}$ in 2 days and $144.7 \mu \mathrm{M}$ in 4 days (not displayed). No statistical comparisons between groups were performed.

\section{Discussion}

This study shows that ammonium accumulates in ready-to-use IVF culture media during storage at $2-8^{\circ} \mathrm{C}$ and during incubation at $37^{\circ} \mathrm{C}$. The main sources are unstable glutamine and the protein supplement.

In the early days of IVF, embryo culture media consisted of simple salt solutions (Lane and Gardner, 2007). Nowadays, most commercially available culture media consist of a mixture including salts, energy sources, proteins, antibiotics, amino acids and vitamins. The different components have been added to the media to support embryo development and implantation rates (Smith et al., 2012). However, it is known that amino acids may spontaneously break down into ammonium, which is toxic for the embryo (Gardner and Lane, 1993).

It was reported that free L-glutamine leads to more ammonium production compared with a dipeptide form of glutamine during incubation (Lane and Gardner, 2003; Tareq et al., 2007; Hashimoto et al., 2008). This study confirms that indeed a solution with L-glutamine results in more ammonium production 
than a glycyl-glutamine solution during incubation and storage. This might explain the significantly higher level of ammonium buildup in the Origio ISM1 medium, since this was the only medium in the present study that contained the unstable L-glutamine at a concentration of $778 \mu \mathrm{M}$ according to Morbeck et al. (2014a). The replacement of L-glutamine with a dipeptide form of glutamine might enhance embryo development (Biggers et al., 2004a; Summers et al., 2005; Hashimoto et al., 2008; Meintjes et al., 2009), although, this was not found by others (Gray et al., 1992).

The commercially available culture media that have been measured in the present study were ready-to-use media, which were already supplemented with protein, and protein-free media, which need to be supplemented with protein before use. We found that there was no detectable ammonium buildup in the protein-free media, while their ready-to-use counterparts showed ammonium buildup. Furthermore, all protein supplements, which can be used to supplement the protein-free media, showed ammonium buildup during incubation. This indicates that the ammonium buildup in the ready-to-use protein supplemented culture media might not only be a result of amino acid degradation, but also the degradation of proteins or of contaminants in the protein supplement (Gray et al., 1992; Chan et al., 1995; Meintjes et al., 2009; Meintjes, 2012; Xiao and Isaacs, 2012; Dyrlund et al., 2014; Morbeck et al., 2014b). Contamination of the protein supplement might play an important role. In a recent study by Morbeck et al. (2014b), it was shown that Irvine SSS contained high levels of both essential and non-essential amino acids, including glutamine, while other protein supplements such as HSA and Sage SPS contained no or very low levels of amino acids. In our study, ammonium buildup during incubation at $37^{\circ} \mathrm{C}$ for 4 days was relatively high in Irvine SSS $(144.7 \mu \mathrm{M})$ when compared with the other protein supplements (ranging from $18.9 \mu \mathrm{M}$ to $31.9 \mu \mathrm{M}$ ).

Apart from the unstable glutamine and protein supplements, also other free amino acids might contribute to the ammonium buildup (Moravek et al., 2012). We did not investigate the deterioration of other components in the media.

In this study, no additional ammonium buildup during incubation at $37^{\circ} \mathrm{C}$ was detected by the presence of an embryo in the medium droplet at Day 3 after ovum retrieval. This seems to be in contrast with a study by Gardner et al. (2001), in which they found an ammonium production up to 30 pmol per embryo per hour for human blastocysts. However, it is not unlikely that the ammonium production of a cleavage stage embryo (consisting of approximately 8 cells) is negligible compared with that of a blastocyst. 
Several studies showed that ammonium has a negative effect on embryo development, pregnancy rates and fetal development in animals (Gardner and Lane, 1993; Lane and Gardner, 1994; Hammon et al., 2000; Lane and Gardner, 2003; Sinawat et al., 2003; Zander et al., 2006; Tareq et al., 2007; Yuan and Krisher, 2010; Gardner et al., 2013) and in humans (Virant-Klun et al., 2006; Hashimoto et al., 2008; Gardner et al., 2013; Li et al., 2013). Most of these studies reported relatively high levels of ammonium in the culture media or added high levels of exogenous ammonium (up to $600 \mu \mathrm{M}$ ). The addition of high levels of exogenous ammonium to culture medium might provide a biased model as the embryos might encounter an artificially high stressful environment (Biggers et al., 2004b). However, in a study by Lane and Gardner (2003), it was found that low levels of exogenous ammonium addition, i.e. 18.8 and $75 \mu \mathrm{M}$, resulted in perturbed metabolism during preimplantation embryo development, lower ICM/total cells ratio and more apoptotic cells in blastocysts and abnormal fetuses. Gardner et al. (2013) also showed in human blastocysts that a gradient of ammonium in culture medium can result in the differential expression of 390 genes involved in metabolism, cell growth and/or maintenance, transcription, cell communication, transport, development and regulation of transcription. The ammonium levels of the ready-to-use embryo culture media from the present study can reach those relatively low levels of ammonium after incubation, especially when they have been stored for several weeks before use.

In line with this, we recently found an inverse association between the age of the embryo culture medium (G-1 PLUS v5) and birth weight of singletons born after fresh embryo transfer (Kleijkers et al., 2015b). It can be deduced that embryos faced a less than optimal environment when cultured in older medium, which might be a result of the ammonium accumulation in the embryo culture media during storage.

With the introduction of time-lapse imaging, new culture media have been developed to support uninterrupted culture of preimplantation embryos (Conaghan, 2014). One of the disadvantages of those continuous single-step media might be the accumulation of toxins, including ammonium (Biggers and Summers, 2008). In the present study, we included Irvine CSC medium and measured comparable levels of ammonium buildup during incubation at $37^{\circ} \mathrm{C}$ as reported by Gilbert et al. (2012). We found no significant differences in ammonium buildup during incubation at $37^{\circ} \mathrm{C}$ between Irvine CSC and the other media from this study, except for some of the cleavage stage media. Recently, in a study comparing the single-step time-lapse medium G-TL and the sequential medium G5 PLUS (G-1 PLUS/G-2 PLUS), low levels $(<35 \mu \mathrm{M})$ of ammonium buildup were measured in both G-TL and G5 PLUS (Hardarson et al., 2015). We did not investigate the ammonium accumulation in G-TL in our study, however, our 
findings for G-1 PLUS and G-2 PLUS were comparable with those from the study by Hardarson et al. (2015).

We have reported that media used for culturing human IVF embryos significantly affected embryo development, pregnancy rates, prenatal and postnatal growth (Dumoulin et al., 2010; Nelissen et al., 2012; Nelissen et al., 2013; Kleijkers et al., 2014). One notable difference, according to the product inserts of the media used during the study period (2003-2006), was the amino acid glutamine; Cook medium contained the free L-glutamine, while Vitrolife medium contained the more stable dipeptide alanyl-glutamine. From the results of the present study, it can be deduced that embryos that were cultured in the Cook medium (with the free L-glutamine), were exposed to higher ammonium levels. It is possible that this resulted in the lower birth weight and postnatal weight of children in the Cook group when compared with children in the Vitrolife group (Dumoulin et al., 2010; Nelissen et al., 2012; Kleijkers et al., 2014).

In conclusion, the main sources of ammonium buildup in ready-to-use culture media are unstable glutamine and the protein supplement. The breakdown of these components into ammonium is more pronounced during incubation at $37^{\circ} \mathrm{C}$, however, it is not negligible during storage at $2-8^{\circ} \mathrm{C}$. This results in increasing ammonium levels in these media over time. Therefore, it is important that the use of free L-glutamine in human embryo culture media is stopped and that the use of protein supplements is thoroughly evaluated. 


\section{CHAPTER 6}

Differences in gene expression profiles between human preimplantation embryos cultured in two different IVF culture media

Sander H.M. Kleijkers, Lars M.T. Eijssen, Edith Coonen, Josien G. Derhaag, Eleni Mantikou, Martijs J. Jonker, Sebastiaan Mastenbroek, Sjoerd Repping, Johannes L.H. Evers, John C.M. Dumoulin, Aafke P.A. van Montfoort

Human Reproduction 2015;30(10):2303-2311 


\section{Abstract}

Study question: Is gene expression in human preimplantation embryos affected by the medium used for embryo culture in vitro during an IVF treatment?

Summary answer: Six days of in vitro culture of human preimplantation embryos resulted in medium-dependent differences in expression level of genes involved in apoptosis, protein degradation, metabolism and cell-cycle regulation.

What is known already: Several human studies have shown an effect of culture medium on embryo development, pregnancy outcome and birth weight. However, the underlying mechanisms in human embryos are still unknown. In animal models of human development, it has been demonstrated that culture of preimplantation embryos in vitro affects gene expression. In humans, it has been found that culture medium affects gene expression of cryopreserved embryos that, after thawing, were cultured in two different media for 2 more days.

Study design, size, duration: In a multicenter trial, women were randomly assigned to two culture medium groups [G5 and human tubal fluid (HTF)]. Data on embryonic development were collected for all embryos. In one center, embryos originating from two pronuclei (2PN) zygotes that were not selected for transfer or cryopreservation on Day 2 or 3 because of lower morphological quality, were cultured until Day 6 and used in this study, if couples consented.

Participants/materials, setting, methods: Ten blastocysts each from the G5 and HTF study groups, matched for fertilization method, maternal age and blastocyst quality, were selected and their mRNA was isolated and amplified. Embryos were examined individually for genome-wide gene expression using Agilent microarrays and PathVisio was used to identify the pathways that showed a culture mediumdependent activity.

Main results and the role of chance: Expression of 951 genes differed significantly $(P<0.01)$ between the G5 and HTF groups. Eighteen pathways, involved in apoptosis, metabolism, protein processing and cell-cycle regulation, showed a significant overrepresentation of differentially expressed genes. The DNA replication, G1 to $\mathrm{S}$ cell-cycle control and oxidative phosphorylation pathways were upregulated in the G5 group compared with the HTF group. This is in agreement with the morphological assessment of the 1527 embryos (originating from 2PN zygotes), which showed that embryos consisted of more cells on Day 2 $(3.73 \pm 1.30$ versus $3.40 \pm 1.35, P<0.001)$ and Day $3(7.00 \pm 2.41$ versus $5.84 \pm$ 2.36, $P<0.001$ ) in the G5 group when compared with the HTF group. Furthermore, the implantation rate was significantly higher in the G5 group 
compared with the HTF group (26.7\% versus $14.7 \%, P=0.002)$ after transfer on the second or the third day after fertilization.

Limitations, reasons for caution: Despite careful matching of the embryos, it cannot be excluded that the differences observed between the study groups are caused by factors that we did not investigate. Extrapolation of these results to embryos used for transfer demands caution as in the present study embryos that were not selected for either embryo transfer or cryopreservation have been used for the culture experiment until Day 6.

Wider implications of the findings: This study shows that gene expression in human preimplantation embryos is altered by the culture medium used during IVF treatment and provides insight into the biological pathways that are affected. Whether these changes in gene expression have any long-term effects on children born after IVF remains unknown. However, it is possible that early adaptations of the preimplantation embryo to its environment persist during fetal and post-natal development.

Study funding/competing interest(s): No funding and no competing interests declared.

Trial registration number: Not applicable. 


\section{Introduction}

Assisted reproductive technology (ART) conceptions are associated with higher risks of obstetric and perinatal complications when compared with spontaneous conceptions (McDonald et al., 2009; McDonald et al., 2010; Pandey et al., 2012; Pinborg et al., 2013). Both subfertility and specific aspects of ART may be risk factors for the adverse perinatal outcome in IVF children (Henningsen and Pinborg, 2014).

The culture medium is one aspect of ART which is considered to be an important factor as it forms the direct environment of the developing preimplantation embryo. It is clear that culture media affect embryo development as well as pregnancy outcome (Mantikou et al., 2013b). Furthermore, our previous studies have demonstrated that culture medium also affects birth weight (Dumoulin et al., 2010; Nelissen et al., 2012). A difference in growth between fetuses derived from embryos cultured in two different culture media was already evident as early as the second trimester of pregnancy and persisted during the first 2 years of post-natal life (Nelissen et al., 2013; Kleijkers et al., 2014). The underlying mechanisms are still unknown, although it has been suggested that gametes and preimplantation embryos adapt to their environment, with long-term consequences (SteegersTheunissen et al., 2013). Although experimental data are virtually absent, these adaptations may act via changes in epigenetic regulation resulting in altered gene expression profiles, either directly or via differences in the proportions of specific cells [i.e. inner cell mass (ICM) and trophectoderm (TE) cells] in the embryo.

Several animal studies did indeed show that gene expression is altered by culture medium, supplementation used or oxygen tension (Khosla et al., 2001a; Fernandez-Gonzalez et al., 2004; Rinaudo and Schultz, 2004; Rinaudo et al., 2006; Saadeldin et al., 2011; Cagnone et al., 2012; Schwarzer et al., 2012; Arias et al., 2013). Furthermore, in an extensive study by Schwarzer et al. (2012), it was found that 13 different human ART culture protocols resulted in distinct cellular and molecular phenotypes in mouse blastocysts. It has been found that certain culture medium components can interfere with the epigenetic regulation of genes. For instance, a side-by-side comparison of five culture media showed that all media had a varying but compromised ability to maintain methylation levels of the imprinted genes H19, Peg3 and Snrpn in comparison with in vivo-derived mouse embryos (Market-Velker et al., 2010).

In humans, it has been reported that culture medium affects gene expression in cryopreserved embryos that, after thawing, were cultured in two different media for 2 more days (from Day 4 to Day 6) (Mantikou et al., 2013a). However, all of these embryos were cultured in the same culture medium before cryopreservation 
(from Day 0 to Day 4), a period during which the embryo is more sensitive to its environment than after compaction (Gardner and Lane, 2005). The aim of the present study was to compare gene expression profiles of human embryos cultured in two different culture media throughout the preimplantation period, thereby increasing our knowledge on pathways involved in human embryonic development in vitro.

\section{Materials and methods}

\section{Study design}

In a Dutch multicenter trial (NTR1979), women were randomly assigned to two culture media groups, with the primary aim to compare live birth rates after embryo transfer on Day 2 or 3 . In one group, all oocytes and resulting embryos were cultured in human tubal fluid (HTF) medium (Lonza, Verviers, Belgium), while in the other group, all oocytes and resulting embryos were cultured in G5 PLUS medium (Vitrolife AB, Göteborg, Sweden). For the present study, at one center (Maastricht University Medical Center) embryos [originating from two pronuclei (2PN) zygotes] not selected for transfer or cryopreservation on Day 2 or 3, were further cultured in G5 or HTF until Day 6 and those that had reached the blastocyst stage were collected.

All participating couples gave written informed consent for the inclusion of their embryos and the use of their data. The study protocol was approved by the Dutch Central Committee on Research involving Human Subjects (CCMO; NL35806. 000.11).

\section{IVF procedures}

Except for the different culture media that are used, all other procedures comprising an IVF treatment, such as ovarian hyperstimulation, follicular aspiration, oocyte fertilization and embryo morphology assessment procedures, were identical in both groups and have been described earlier (Kleijkers et al., 2014). Briefly, patients received long down-regulation with $\mathrm{GnRH}$ agonists and underwent ovarian stimulation with recombinant FSH. Thirty-six hours after hCG injection, ultrasound-guided oocyte retrieval was performed and progesterone was administered vaginally for luteal phase support. In case of a pregnancy, progesterone administration was extended for 3 weeks.

HTF medium is used in a monoculture system and consists of a balanced salt solution with added carbohydrates, glucose, pyruvate and lactate. HTF medium 
was supplemented with $10 \%$ human serum albumin (HSA) (Albuman: Sanquin, Amsterdam, the Netherlands) and embryos were cultured in a $5 \% \mathrm{CO}_{2}$ and $5 \% \mathrm{O}_{2}$ environment. G5 PLUS media are used in a sequential culture system (G-IVF PLUS, G-1 PLUS and G-2 PLUS), involving the sequential culture of embryos in media of different composition. G-IVF PLUS is used during IVF, G-1 PLUS is used for culture of embryos from the pronuclear stage (Day 1 ) to early Day 3, while G-2 PLUS is used for culture of embryos from Day 3 to the blastocyst stage. G-1 PLUS and G-2 PLUS are ready-to-use media already supplemented with $5 \%$ human serum albumin (HSA) and G5 PLUS embryos were cultured in a $6 \% \mathrm{CO}_{2}$ and $5 \% \mathrm{O}_{2}$ environment. Apart from the balanced salt solutions and energy sources, the G5 media also contain other components, including amino acids and vitamins. The complete list of components in the media used is shown in Supplementary Table S1 (available online). To align HTF and G5 methodology, embryos in the HTF group were transferred to a fresh culture medium droplet on Day 3. Embryos were cultured individually and culture droplets were covered by mineral oil (Irvine Scientific, Santa Ana, CA, USA).

Embryo development was recorded daily. Embryo transfer was performed on Day 2 or 3 after oocyte retrieval and our standard transfer policy consisted of single embryo transfer for women under 38 years old with at least one good quality embryo available. In all other cases, two embryos were transferred.

\section{Sample collection and selection}

On Day 6, individual embryos were transferred from the culture medium into a droplet of protease solution ( $500 \mathrm{U} / \mathrm{ml}$; Sigma-Aldrich, St. Louis, MO, USA) until the zona pellucida had dissolved and were then rinsed in two droplets of Dulbecco's phosphate-buffered saline (Life Technologies, Carlsbad, CA, USA). Each embryo was transferred into a separate microcentrifuge tube with $3 \mu$ of ice-cold lysis buffer [0.8\% Igepal (Sigma-Aldrich), 1 IU/ $\mu$ I SUPERase-In (Life Technologies), 5 $\mathrm{mmol} / \mathrm{l}$ dithiothreitol (Life Technologies)], flash-frozen in liquid nitrogen and stored at $-80^{\circ} \mathrm{C}$ until RNA isolation. Ten blastocysts from each study group, matched for fertilization method (IVF or ICSI), maternal age (age difference of $<3$ years) and blastocyst quality were selected for mRNA analysis. Blastocysts were scored according to the Gardner grading system (Gardner et al., 2004). Briefly, blastocysts were scored according to size (1-6: from early blastocyst to hatched blastocyst), ICM (A: tightly packed, many cells; B: loosely grouped, several cells; C: very few cells) and TE (A: many cells forming a tightly knit epithelium; $B$ : few cells; $\mathrm{C}$ : very few cells forming a loose epithelium). To prevent any genetic bias that might arise by adding multiple embryos from one couple into one group, only one blastocyst per couple was included in the study. 
Sample preparation was performed according to a recently developed protocol by the MicroArray Department of the University of Amsterdam, validated for analysis of very small amounts (picograms) of RNA (submitted data). RNA was isolated with the Arcturus PicoPure RNA Isolation Kit (Life Technologies) following the instructions of the manufacturer with minor adaptations. RNA extraction was performed with $50 \mu \mathrm{l}$ Extraction Buffer and the recommended DNase treatment was performed. RNA was amplified using the Arcturus RiboAmp HS Plus RNA Amplification Kit according to the manufacturer's instructions. The quality of the amplified RNA (aRNA) was verified using the 2100 Bioanalyzer System with the RNA Nano 6000 Kit (Agilent Technologies, Santa Clara, CA, USA) and the concentration was measured on a Nanodrop ND-1000 spectrophotometer (Thermo Fisher Scientific, Waltham, MA, USA).

\section{Microarray hybridization}

Five micrograms of aRNA was dried in a speedvac, then dissolved in $5 \mu \mathrm{l} 50 \mathrm{mM}$ Carbonate Buffer ( $\mathrm{pH} \mathrm{8.5)} \mathrm{and} \mathrm{incubated} \mathrm{at} 37^{\circ} \mathrm{C}$ for 10 minutes. Ten microliters of CyDye diluted in dimethylsulphoxide was added to each sample, followed by incubation in the dark at room temperature for 1 hour. Cy3 was used to label the individual embryo samples and Cy5 was used for a reference sample (comprising a pool of six samples). To quench the reactions, samples were incubated with $5 \mu$ of $4 \mathrm{M}$ hydroxylamine at room temperature for 15 minutes. The labeled RNA was purified using the MicroElute RNA Clean Up Kit (Omega Bio-Tek, Norcross, GA, USA) and finally eluted in $15 \mu \mathrm{l}$ RNase/DNase free water. The yield and CyDye incorporation of labeled aRNA were measured with a Nanodrop ND-1000 spectrophotometer.

Fragmentation and hybridization was performed according to the Two-Color Microarray-Based Gene Expression Analysis protocol (Agilent Technologies). A total of $825 \mathrm{ng}$ of Cy3 labeled sample aRNA and $825 \mathrm{ng}$ of Cy5 labeled reference aRNA were added to the fragmentation mix and samples were incubated at $60^{\circ} \mathrm{C}$ for 30 minutes. After addition of the hybridization mix, samples were hybridized to the $180 \mathrm{k}$ custom designed Agilent arrays (Agilent technologies; design ID:028004) for 17 hours at $65^{\circ} \mathrm{C}$ and $10 \mathrm{rpm}$. The $180 \mathrm{k}$ microarrays contained almost 42,000 probes in quadruplet and 11,200 control probes. The microarray slides were washed and scanned in an ozone-free room using a Microarray scanner GA2505C (Agilent Technologies). Slides were scanned at $3 \mu \mathrm{m}$ resolution and the microarray data were extracted with Feature Extraction software 10.7.1 (Agilent Technologies). 


\section{Data analysis}

The differences in embryo development and implantation rate between study groups were tested by Student's t-test and $\chi^{2}$ test, respectively. Analyses were conducted with IBM SPSS Statistics 20 (IBM, Armonk, NY, USA) and two-sided Pvalues of $<0.05$ were considered to reflect statistical significance. Microarray data analysis was performed using the R statistical software (http://cran.r-project.org/). All arrays were subjected to a variety of quality control checks (box and density plots of signals, MA plots, virtual 2D array representations, correlation plots, among others) related to sample quality, hybridization quality and signal comparability and data were normalized using LOESS (local regression smoothing) normalization. Low quality spots were discarded and values for replicate probes (each probe was present in quadruplicate) summarized by averaging. Unexpressed probes ( $<5$ expression value, ${ }^{2}$ log scale) and probes with too many missing values (>5 x 4 missing) were excluded from further analysis. Linear modeling using the Limma package (Gentleman et al., 2004; Smyth, 2005) was conducted to compute the genes that had significantly (as defined by a $P$-value $<0.01$ ) changed between study groups by fitting an interaction model with culture medium and fertilization method. For the biological interpretation of the gene expression data, PathVisio 3.1.3 (http://www.pathvisio.org/) with the identifier mapping Hs_Derby_20130701 bridge file was used to map the Ensembl IDs (Flicek et al., 2013) of measured genes to biological pathways from WikiPathways (van lersel et al., 2008) and to generate a list of Z-score ranked pathways based on the (over)representation of differentially expressed genes (DEGs) within each pathway. All pathways with a Zscore of 1.96 or higher were included in the biological interpretation, reflecting a significance level of 0.05 . Microarray data are available in the ArrayExpress database (Kolesnikov et al., 2015) under accession number E-MTAB-3405.

\section{Results}

\section{Sample matching and processing}

Twenty blastocysts, 10 from the G5 and 10 from the HTF group, were selected for microarray analysis (Table 1). They were matched based on blastocyst quality, fertilization method and maternal age. Blastocyst quality and fertilization method were identical within pairs and there was a maximal difference of 2.8 years in maternal age within matched pairs. Characteristics of the selected samples from both embryo culture medium groups (G5 and HTF) are presented in Table 2. Amplification was successful for all samples and all 20 arrays passed the quality control for sample quality, hybridization quality and signal comparability. Signals after LOESS normalization were very comparable. 


\section{Microarray data analysis}

The microarrays contained 42,405 probes covering 23,645 genes in total and overall 28,399 probes corresponding to 17,692 genes were detected after exclusion of unexpressed probes and probes with too many missing values. The expression of 951 genes differed significantly $(P<0.01)$ between human preimplantation embryos that had been cultured in G5 or HTF medium and those are presented in Supplementary Table S2 (available online). There were 544 (57.2\%) significantly upregulated and 407 (42.8\%) significantly downregulated genes in the G5 group compared with the HTF group. Of these 951 DEGs, 389 genes showed a fold change of $<-2$ or $>2$ and 81 genes showed a fold change of $<-5$ or $>5$.

Table 1. Characteristics of the matched human embryo samples from the two study groups.

\begin{tabular}{llll} 
Medium & Blastocyst quality ${ }^{1,2,3}$ & Fertilization method & Maternal age \\
\hline G5 & 4AA & IVF & 33.8 \\
HTF & & IVF & 33.4 \\
G5 & 4AA & & 33.5 \\
HTF & & IVF & 33.0 \\
G5 & 4BA & & 30.7 \\
HTF & & ICSI & 31.9 \\
G5 & & 27.8 \\
HTF & 4BA & 25.6 \\
G5 & & IVF & 33.6 \\
HTF & & 35.9 \\
G5 & 4BA & ICSI & 34.0 \\
HTF & & & 34.3 \\
G5 & 4CA & ICSI & 31.3 \\
HTF & & & 30.7 \\
G5 & 3AA & ICSI & 32.6 \\
HTF & & & 31.9 \\
G5 & 3BA & IVF & 34.8 \\
HTF & & & 37.6 \\
G5 & 3BA & & 33.6 \\
HTF & & & 32.2 \\
\hline
\end{tabular}

Blastocysts were scored according to the Gardner grading system.

${ }^{1}$ Size score: 1 = early blastocyst; 2 = blastocyst; 3 = full blastocyst; 4 = expanded blastocyst; 5 = hatching blastocyst; 6 = hatched blastocyst.

${ }^{2}$ ICM score: $A$ = tightly packed, many cells; $B=$ loosely grouped, several cells; $C=$ very few cells.

${ }^{3}$ TE score: $A=$ many cells forming a tightly knit epithelium; $B=$ few cells; $C=$ very few cells forming a loose epithelium.

For each of the 951 DEGs, the number of matched pairs of blastocysts that showed a change in level of mRNA expression in the same direction (up- or downregulation in G5) was calculated (Figure 1). For any one gene, there was 
always at least 6 pairs that changed in the same direction. Eighty-nine percent (849/951) of all DEGs showed a change in the same direction in at least 8 of the 10 matched pairs, while this was $90 \%$ (349/389) and 86\% (70/81) for genes with a fold change of at least 2 -fold and 5 -fold, respectively. The 70 DEGs that are similarly up- or downregulated in at least 8 matched pairs with a fold change of at least 5-fold are shown in Table 3.

Table 2. Characteristics of the selected samples for this study.

\begin{tabular}{lcc}
\hline & $\mathrm{G} 5(\mathrm{n}=10)$ & HTF $(\mathrm{n}=10)$ \\
Fertilization method & & $5(50)$ \\
IVF & $5(50)$ & $5(50)$ \\
ICSI & $5(50)$ & $32.7 \pm 1.0$ \\
Maternal age (years) & $32.6 \pm 0.7$ & $38.1 \pm 1.9$ \\
Paternal age (years) & $35.8 \pm 2.1$ & $3.0 \pm 0.5$ \\
Duration of subfertility (years) & $3.0 \pm 0.4$ & $2(20)$ \\
Primary indication for IVF/ICSI & & $1(10)$ \\
$\quad$ Tubal & $2(20)$ & $6(60)$ \\
$\quad$ Endometriosis & $2(20)$ & $1(10)$ \\
$\quad$ Male subfertility & $5(50)$ & $3.4 \pm 0.5$ \\
$\quad$ Unexplained & $1(10)$ & $17.5 \pm 3.8$ \\
Number of cells on Day 2 & $3.2 \pm 0.5$ & $7.4 \pm 0.6$ \\
Fragmentation on Day 2 (\%) & $11.0 \pm 3.8$ & $18.0 \pm 2.9$ \\
Number of cells on Day 3 & $7.6 \pm 0.6$ & \\
Fragmentation on Day 3 (\%) & $18.7 \pm 4.8$ & \\
\hline
\end{tabular}

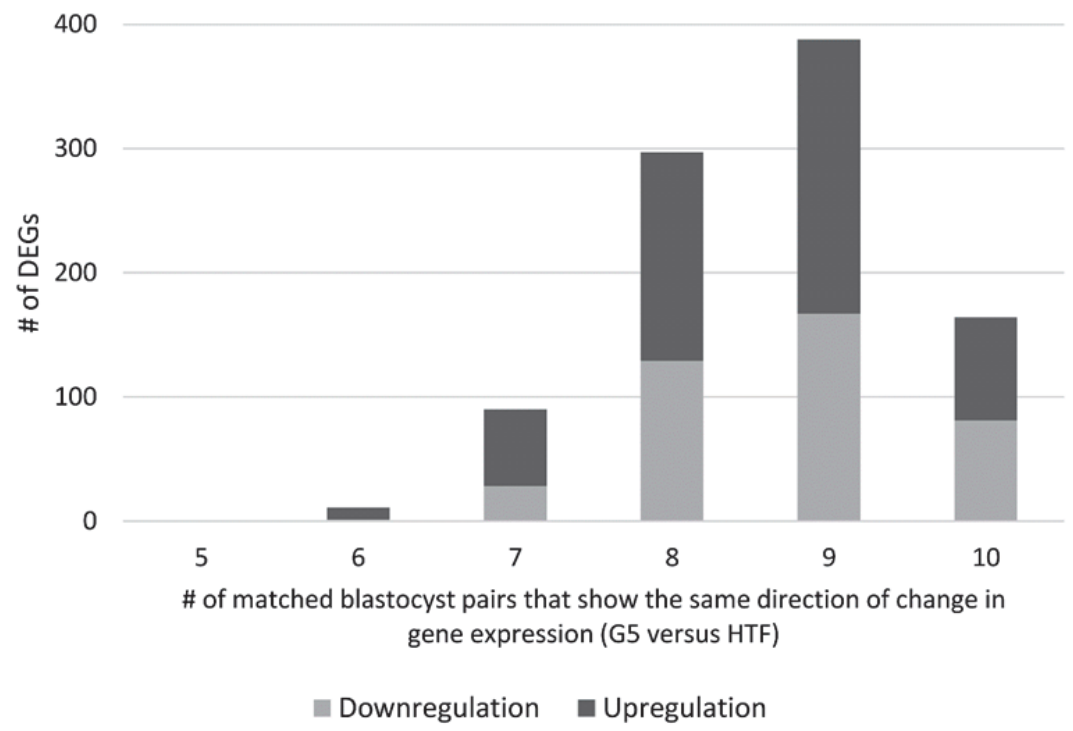

Figure 1. Number of differentially expressed genes (DEG) according to the number of matched blastocyst pairs that show the same direction of change in gene expression (up- or down-regulation in G5 when compared with HTF) for a gene. 
IVF culture medium and human embryo gene expression

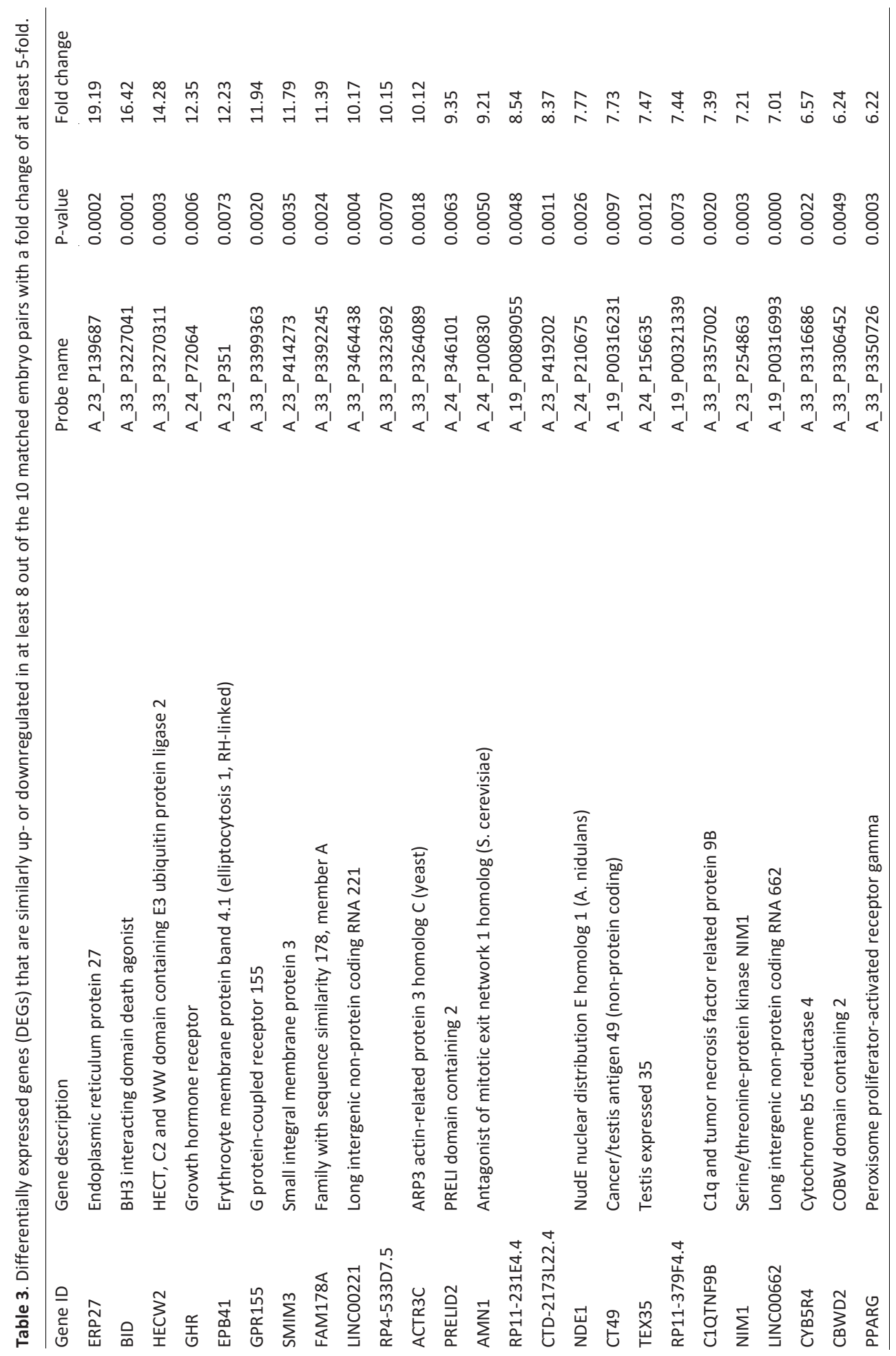




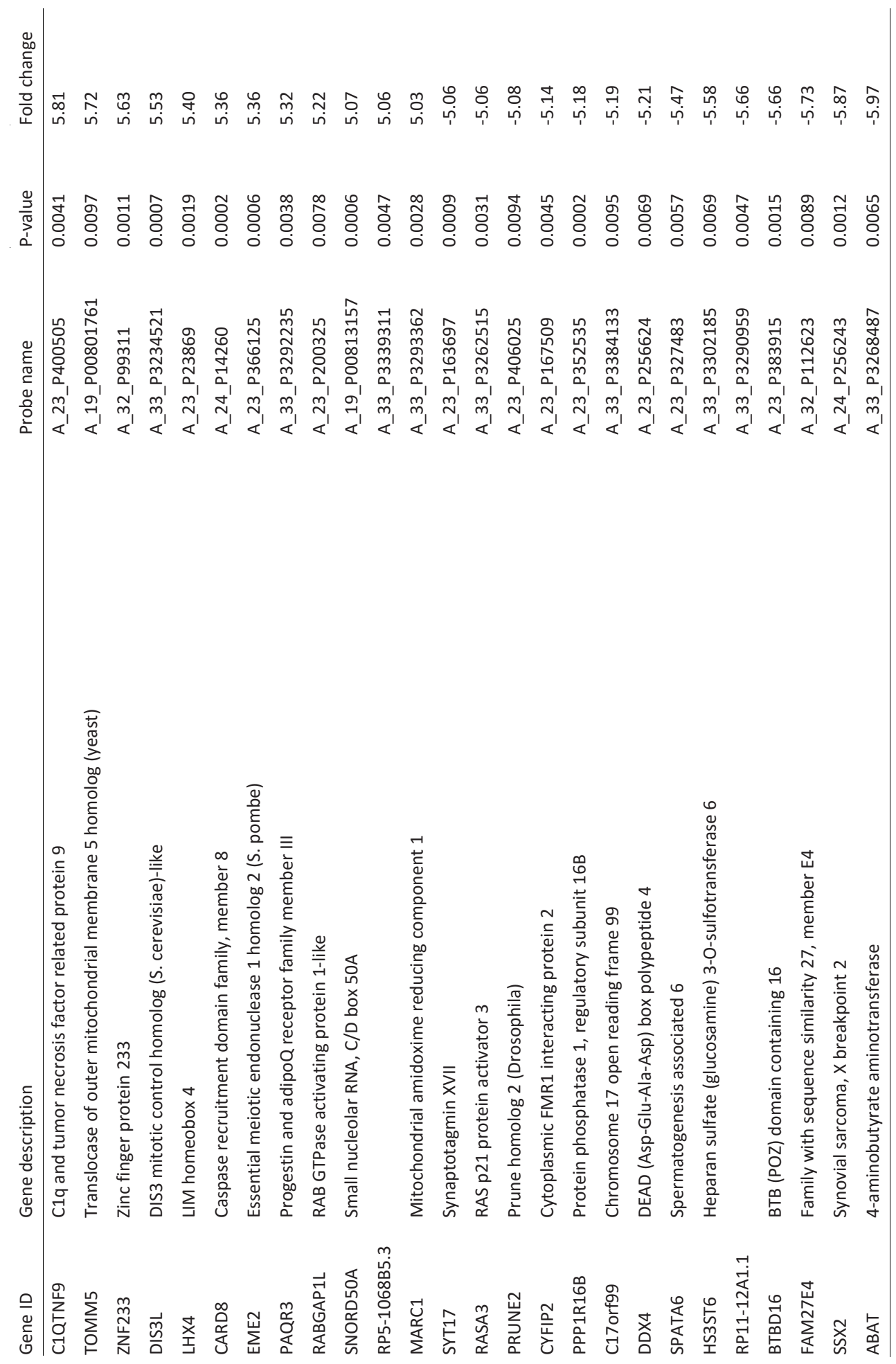


IVF culture medium and human embryo gene expression

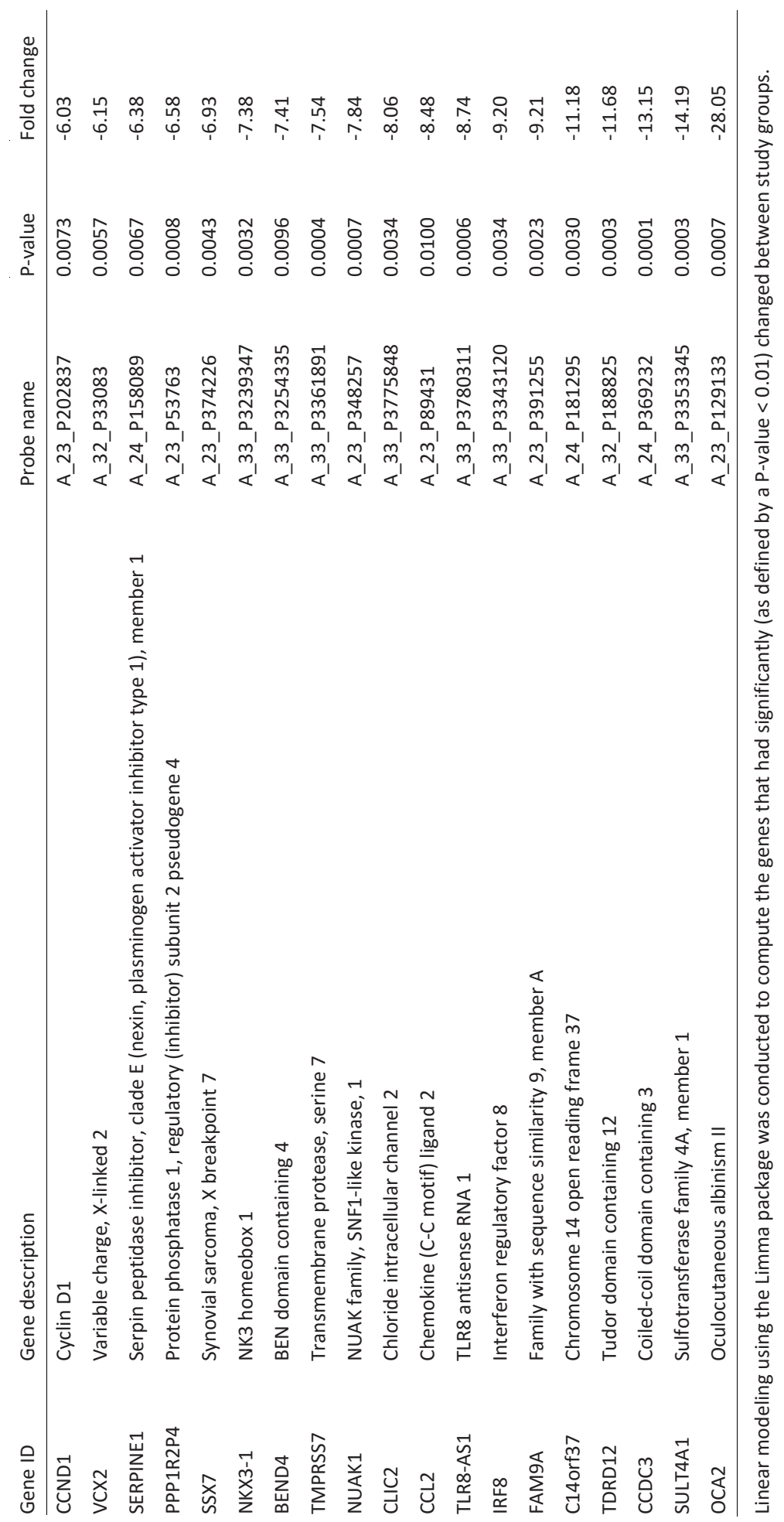


Pathway analysis revealed 18 biological pathways with overrepresentation of DEGs, involved in apoptosis, protein degradation, metabolism and cell cycle regulation pathways (Table 4). All DEGs of the DNA replication pathway, 7 out of 9 in the G1 to $S$ cell cycle control pathway and 6 of the 7 in the oxidative phosphorylation pathway were upregulated in the G5 group compared with the HTF group. Furthermore, all DEGs that code for subunits of the Ubiquitin, Proteasome $26 \mathrm{~S}$ and Proteasome $20 \mathrm{~S}$ complexes from the proteasome degradation pathway were upregulated in the G5 group compared with the HTF group. There was no uniform up- or downregulation of DEGs in the other biological pathways.

Table 4. Pathways with a significant overrepresentation of DEGs clustered per biological process.

\begin{tabular}{|c|c|c|c|c|c|}
\hline Pathway & $\begin{array}{l}\text { Positive } \\
\text { (r genes) }\end{array}$ & $\begin{array}{l}\text { Measured } \\
\text { (n genes) }\end{array}$ & $\begin{array}{l}\text { Total } \\
\text { genes }\end{array}$ & $\%$ & Z-score \\
\hline \multicolumn{6}{|l|}{ Cell cycle regulation } \\
\hline RB in Cancer & 20 & 95 & 104 & $21.05 \%$ & 5.66 \\
\hline Gastric cancer network 2 & 5 & 25 & 33 & $20.00 \%$ & 2.67 \\
\hline G1 to $\mathrm{S}$ cell cycle control & 9 & 62 & 69 & $14.52 \%$ & 2.48 \\
\hline DNA Replication & 6 & 38 & 50 & $15.79 \%$ & 2.25 \\
\hline \multicolumn{6}{|l|}{ Apoptosis } \\
\hline Apoptosis & 11 & 73 & 85 & $15.07 \%$ & 2.88 \\
\hline TP53 Network & 4 & 17 & 23 & $23.53 \%$ & 2.78 \\
\hline DNA Damage Response & 9 & 61 & 75 & $14.75 \%$ & 2.53 \\
\hline DNA Damage Response (only ATM dependent) & 11 & 85 & 101 & $12.94 \%$ & 2.33 \\
\hline miRNA Regulation of DNA Damage Response & 9 & 66 & 106 & $13.64 \%$ & 2.27 \\
\hline \multicolumn{6}{|l|}{ Protein processing } \\
\hline Parkin-Ubiquitin Proteasomal System pathway & 9 & 58 & 75 & $15.52 \%$ & 2.70 \\
\hline Proteasome Degradation & 8 & 54 & 67 & $14.81 \%$ & 2.40 \\
\hline \multicolumn{6}{|l|}{ Metabolism } \\
\hline Glycerophospholipid Biosynthetic Pathway & 6 & 23 & 94 & $26.09 \%$ & 3.73 \\
\hline Folate Metabolism & 8 & 50 & 139 & $16.00 \%$ & 2.65 \\
\hline Vitamin B12 Metabolism & 6 & 36 & 118 & $16.67 \%$ & 2.40 \\
\hline SREBF and miR33 in cholesterol and lipid homeostasis & 3 & 15 & 19 & $20.00 \%$ & 2.06 \\
\hline Nuclear Receptors in Lipid Metabolism and Toxicity & 4 & 23 & 45 & $17.39 \%$ & 2.06 \\
\hline Oxidative phosphorylation & 7 & 52 & 69 & $13.46 \%$ & 1.96 \\
\hline \multicolumn{6}{|l|}{ Development } \\
\hline Hedgehog Signaling Pathway & 3 & 13 & 17 & $23.08 \%$ & 2.37 \\
\hline
\end{tabular}

RB, retinoblastoma; TP53, tumor protein p53; ATM, ATM serine/threonine kinase; miRNA, microRNA; SREBF, sterol regulatory element-binding protein gene; miR33, microRNA 33. 
Sex of the 20 embryos were determined by evaluating the expression of six Yspecific genes (USP9Y; ubiquitin specific peptidase 9, Y-linked, DDX3Y; DEAD (AspGlu-Ala-Asp) box helicase 3, Y-linked, RPS4Y1; ribosomal protein S4, Y-linked 1, RPS4Y2; ribosomal protein S4, Y-linked 2, EIF1AY; eukaryotic translation initiation factor $1 A, Y$-linked and ZFY; zinc finger protein, $Y$-linked). Nine embryos were identified as female, while 11 embryos were identified as male. The interaction of culture medium and sex was investigated by linear modeling and only 137 genes had a significant interaction term $(P<0.01)$, which is lower than would be expected by chance. Pathway analysis showed no overrepresentation of those genes in specific pathways. These findings suggest no difference in effect of the culture medium on gene expression between the sexes.

\section{Embryo development and implantation}

To investigate whether the G5 upregulated pathways involved in cell cycle and metabolism were related to morphological differences, data on embryo development and implantation were collected. Morphological assessment of the 1527 embryos (originating from 2PN zygotes) showed that embryos consisted of more cells on Day $2(3.73 \pm 1.30$ versus $3.40 \pm 1.35, P<0.001)$ and Day $3(7.00 \pm$ 2.41 versus $5.84 \pm 2.36, P<0.001$ ) in the $\mathrm{G} 5$ group when compared with the HTF group. Furthermore, the implantation rate was significantly higher in the $\mathrm{G} 5$ group compared with the HTF group $(26.7 \%$ versus $14.7 \%, P=0.002)$ after transfers performed at either the second or the third day after fertilization.

\section{Discussion}

This study using embryos that were not transferred shows that culture of human preimplantation embryos for 6 days resulted in a medium-dependent changes in expression level of genes involved in apoptosis, protein degradation, metabolism and cell cycle regulation.

The material used in this study (i.e. human embryos) has a limited availability, restricting the number of analyses. Therefore, we mainly focused on investigating pathways that differed between the two study groups, instead of individual genes. Although quantitative PCR validation was not performed, combining the measurements from all the genes in a pathway and not focusing on exact fold changes of specific genes but on overall direction of change in the pathway is likely to make the analysis more robust and less sensitive for potential chance findings. Pathway analysis showed overrepresentation of upregulated DEGs in DNA replication, $\mathrm{G} 1$ to $\mathrm{S}$ cell cycle control and oxidative phosphorylation pathways in the G5 group compared with the HTF group. This indicates increased 
cell proliferation in embryos of the G5 group, which is in line with the observations from the morphological assessment of these embryos that they consist of relatively more cells on Day 2 and 3, compared with the HTF group. Furthermore, the upregulation of genes involved in oxidative phosphorylation is thought to positively affect human oocyte quality and embryo development (Hsieh et al., 2004). It is therefore not unlikely that the differences in gene expression profiles were already introduced during earlier stages of preimplantation development. All DEGs that code for subunits of the Ubiquitin, Proteasome 26S and Proteasome 20S complexes from the proteasome degradation pathway were upregulated in the G5 group compared with the HTF group. These complexes are involved in degradation, cellular relocation, activity and interactions of proteins (Fang and Weissman, 2004). Inhibition of the ubiquitin-proteasome pathway (UPP) reduces the implantation potential in mouse embryos (Wang et al., 2004). It is possible that the downregulation of the complexes involved in this pathway is indicative for the observed lower implantation rate in the HTF group.

Not only proliferation and differentiation are important for the development of the preimplantation embryo, but also apoptosis is essential as it mediates the elimination of misplaced, dysfunctional or unneeded cells (Huppertz and Herrler, 2005). In the present study, 11 genes that mapped to the apoptosis pathway were differently expressed between the G5 and HTF groups. However, there was no clear up- or downregulation of anti- and pro-apoptotic genes. This might be the result of the complex balanced regulation of both anti- and pro-apoptotic genes in this pathway (Wang et al., 2009).

Although the exact concentrations of the different components in G5 are unknown, the major differences in composition between G5 and HTF are the albumin source and the addition of amino acids, vitamins, hyaluronan and lipoic acid in G5. Although amino acids are not required for the development of zygotes into blastocysts, it is well established that the addition of amino acids has a beneficial effect on preimplantation embryo development in several species (Gardner et al., 2000; Biggers and Summers, 2008). It has been shown that the cellular concentration of amino acids can regulate the expression of numerous genes in different mammalian cell lines (Jousse et al., 2004). In a study by Rinaudo and Schultz (2004), the global gene expression patterns of mouse blastocysts that were cultured in either Whitten's medium (WM) or K-modified simplex optimized medium + amino acids (KSOM/AA) were compared with those of blastocysts that developed in vivo. Culture in KSOM/AA resulted in gene expression patterns that resembled the patterns of the in vivo counterparts more closely than culture in WM (which lacks amino acids). Affected biological and molecular processes after culture in WM included ribosome biogenesis and protein synthesis, cell 
organization and biogenesis, apoptosis, regulation of cell cycle and proliferation, and selenium binding. In the present study, that compared the expression profiles of human blastocysts after culture in medium with (G5) or without (HTF) amino acids, a differential expression of genes involved in apoptosis, protein processing, metabolism and cell-cycle regulation was found.

Patterns of gene expression change during human preimplantation embryo development from oocyte to blastocyst (Assou et al., 2011; Vassena et al., 2011; Mantikou et al., 2013a; Shaw et al., 2013; Xue et al., 2013). For example, in a recent study by Yan et al. (2013) in which global gene expression patterns were investigated during seven consecutive stages of human preimplantation development by single-cell RNA-Seq profiling, it was found that embryo gene expression profiles differ significantly at different stages of development. Factors related to the IVF treatment or patient can also have an effect on gene expression in preimplantation embryos. For example, maternal age affects gene expression in oocytes (Steuerwald et al., 2007; Grondahl et al., 2010), morulas and blastocysts in humans (Mantikou et al., 2013a). In mice, it was found that the method of fertilization (IVF or ICSI) affected gene expression in blastocysts (Giritharan et al., 2010; Bridges et al., 2011). To reduce any bias on the outcomes in our study caused by these factors, samples were matched for blastocyst quality, fertilization method and maternal age. Despite careful matching of the embryos, it cannot be ruled out that the observed differences between the G5 and HTF groups are caused or affected by factors not investigated in this study. Furthermore, the altered gene expression profiles might be a result of differences in the proportion of specific cells in the embryos from both groups (i.e. proportion of ICM and TE cells).

In the present study, we used embryos that were not selected for transfer or cryopreservation on Day 2 or 3 and these embryos were cultured until Day 6 . Those embryos were compromised at early developmental stages compared with the embryos that were selected for the IVF treatment. Therefore, it is appropriate to be cautious when extrapolating these results to good quality embryos. However, findings are in line with the results of a study in which gene expression profiles of frozen-thawed embryos that had been cultured in either G5 or HTF medium for 2 more days (from Day 4 to Day 6) were compared (Mantikou et al., 2013a). Analysis of these good quality embryos also showed differential expression of genes related to metabolism, cell-cycle processes and oxidative phosphorylation.

In conclusion, gene expression in spare human preimplantation embryos is changed by the culture medium used during an IVF treatment. Furthermore, several biological pathways showed a significant overrepresentation of DEGs and 
some pathways involved in proliferation were upregulated in G5 medium compared with HTF medium. These results correlate with the findings from the morphological assessment of all embryos, which showed that embryos in the G5 group consisted of more cells on Days 2 and 3 . Whether these observed differences are associated with long-term effects of culture media on fetuses and children remains as yet unknown. However, it is not unlikely that early adaptations of the preimplantation embryo to its environment persist during fetal and post-natal development. 


\section{CHAPTER 7}

General discussion and future prospects 


\section{General discussion}

The DOHaD hypothesis proposes that the origin of later health is programmed during early life (conception, pregnancy and infancy) under the influence of environmental factors. During IVF treatments, the environment of the gametes, embryos and fetuses is suboptimal and may result in the adverse perinatal outcome observed in IVF children (as described in the introduction). However, it is as yet unknown which aspects of ART are responsible for the poorer outcome. The goal of this thesis was to assess the effect of embryo culture media on perinatal and early postnatal outcome. Furthermore, we investigated which components of the culture media might play a role in determining the outcome and what the possible mode of action could be, by investigating gene expression profiles.

\section{Embryo culture media and early health outcome}

From studies in animal models, it is clear that culture medium constituents can have profound effects on the perinatal and postnatal outcome of the offspring. In previous studies in humans, some indicated that there was an effect of culture medium on birth weight (Dumoulin et al., 2010; Nelissen et al., 2012; Eskild et al., 2013; Hassani et al., 2013; Zhu et al., 2014a), while others found no such effect (Eaton et al., 2012; Vergouw et al., 2012; Carrasco et al., 2013; Lin et al., 2013; Ziebe et al., 2013; Lemmen et al., 2014; Wunder et al., 2014; De Vos et al., 2015; Yin et al., 2015; Bouillon et al., 2016) [see review by Zandstra et al. (2015)]. However, these (observational) studies were limited by several features, such as a non-randomized design, retrospective design, use of different culture media over consecutive periods of time, use of historical controls, and/or small sample sizes. The association between culture medium and outcome in human IVF offspring is thus far not conclusive. Therefore, we conducted a large multicenter, randomized, double-blind trial comparing live birth rates as well as pregnancy and perinatal outcomes after IVF using two culture media that have been used worldwide in IVF programs, HTF medium and Vitrolife G5 medium. We confirmed that embryo culture medium affects birth weight in humans and we found a significant mean difference of $158 \mathrm{~g}$ between the two culture medium groups. Furthermore, in our follow-up study, we investigated if the difference in weight at birth between the singletons from our previous studies (Dumoulin et al., 2010; Nelissen et al., 2012) would disappear, persist or increase during the first 2 years of their lives. We found that the difference in weight persisted; the in vitro culture of human embryos in medium from Cook resulted in singletons with a lower weight during the first 2 years of life compared with singletons born after embryo culture in medium from Vitrolife G3. Recently, a small study investigating the postnatal 
development of children after the use of either Global medium or Single Step Medium (SSM) in 371 IVF cycles was published (Bouillon et al., 2016). This randomized study was stopped prematurely as the pregnancy rates were significantly lower in the SSM group. Despite of the small sample size (Global: 31 children; SSM: 24 children), it was found that children from the Global group obtained better developmental scores (including social skills, self-help, gross and fine motor skills, expressive language, language comprehension, as well as letter and number knowledge) than those from the SSM group at the age of 5 years. Those human studies, together with the studies using animal models (as described in the introduction), imply that ART-procedure related factors may be, at least in part, the cause of the adverse perinatal and early postnatal outcome.

\section{Embryo culture media and suboptimal environment}

It is worthwhile to investigate which components are responsible for the differences in outcome. Nowadays, most embryo culture media share the same basic components such as a buffer system, mineral salts, carbohydrates and amino acids and most of them are supplemented with albumin and antioxidants. However, it is difficult to assign the observed effects to specific components in the embryo culture media, as comparisons between the studies is complicated by several methodological issues and by the differences in amino acids or carbohydrate compositions, addition of other components, and by the lack of information on the exact amount of each component in the culture media. The different components have been added to the media to support embryo development and implantation rates (Smith et al., 2012). However, our studies along with other studies have shown that current culture media used in mammalian ART remain suboptimal, as they play a role in the adverse outcome in ART singletons.

Amino acids play an important role in embryo development via homeostatic regulation and a wide variety in type of - and amount of - amino acids added is observed within the different culture media (Chronopoulou and Harper, 2015). Studies in human and animal models have shown that the in vitro development of embryos can be improved by the addition of amino acids (Liu and Foote, 1995; Devreker et al., 1998; Lane and Gardner, 1998; Summers and Biggers, 2003). Lane and Gardner (1994) have shown in a mouse model that fetal weight was affected by the composition of amino acids in the culture medium. In our RCT, one of the notable differences between the embryo culture media was that the Vitrolife G5 medium contains certain amino acids and that the HTF medium completely lacks amino acids. In a retrospective study by Vergouw et al. (2012), Sage Quinn's Advantage Protein Plus medium was compared with HTF medium. Sage Quinn's Advantage Protein Plus medium also contains amino acids and although no 
significant differences in birth weight between the groups were found, the same trend was seen with children from the HTF group being heavier than children from the other group.

It is known that amino acids, notably free L-glutamine, can spontaneously break down into ammonium, which is toxic for the embryo (Gardner and Lane, 1993). We confirmed that medium containing free L-glutamine results in more ammonium than a medium containing glycyl-glutamine, both during incubation and storage. According to the product inserts of the media used during our previous studies, there was a difference in the glutamine source between the culture media; Cook medium contained the free L-Glutamine, while Vitrolife medium contained the more stable dipeptide alanyl-glutamine. It can be deduced that embryos that were cultured in Cook medium (with the free L-Glutamine), were exposed to higher ammonium levels, which might result in the lower birth and postnatal weight of children in the Cook medium group when compared with children in the Vitrolife medium group. Eskild et al. (2013) have found a comparable difference, with children born after culture in Medicult (with the free L-Glutamine) having a lower birth weight than children cultured in Vitrolife (stabilized glutamine). An opposite effect, with Medicult resulting in a higher birth weight as compared with Vitrolife, was observed by Hassani et al. (2013). However, their study had several limitations such as no correction for any confounding factors.

We also identified the protein supplement as a source for the ammonium accumulation. It has been suggested that the protein source in embryo culture media affects birth weight, as differences in gestational age- and gender-adjusted birth weight and the proportion of large-for-gestational age babies have been observed between embryos cultured in G1 v5 media freshly supplemented with 5 $\mathrm{mg} / \mathrm{ml}$ pharmaceutical human serum albumin solution and those cultured in ready-to-use, pre-supplemented, G1-PLUS v5 media (Zhu et al., 2014a). In our $\mathrm{RCT}$, the source of the albumin supplements differed between the study groups, which might have resulted in the differences in birth weight. Furthermore, we found that birth weight of singletons born after IVF showed an inverse association with age of the embryo culture medium (G1-PLUS v5). This supports the contention that embryos face a less than optimal environment when cultured in older medium. This might be a result of the ammonium accumulation in the embryo culture media during storage as a result of deterioration of medium components. This study provides another indication that the embryonic environment affects fetal growth. 
The mechanisms by which 2-3 days of embryo culture might affect birth weight nine months later are not exactly known, but might be mediated through an effect on the epigenome of the developing preimplantation embryo (van Montfoort et al., 2012). In animal models, it has indeed been found that culture medium components can interfere with the epigenetic regulation of genes (Doherty et al., 2000; Khosla et al., 2001a; Mann et al., 2004; Market-Velker et al., 2010) and that gene expression is dependent on the embryo culture medium or supplementation used (Khosla et al., 2001a; Fernandez-Gonzalez et al., 2004; Rinaudo and Schultz, 2004; Saadeldin et al., 2011; Cagnone et al., 2012; Schwarzer et al., 2012; Arias et al., 2013). Aberrant fetal growth and development was found after culturing mouse embryos in medium supplemented with serum via the regulation of multiple growth-related imprinted genes in the fetuses (Khosla et al., 2001a). Exposure to low levels of ammonium generated morphologically normalappearing blastocysts that were compromised in the expression of the imprinted growth regulator gene $\mathrm{H} 19$ and future fetal development (Lane and Gardner, 2003). Altered insulin-like growth factor 1 receptor (IGF1R) expression in mouse embryos was observed after the exposure to palmitic acid, which resulted in smaller pups at delivery (Jungheim et al., 2011). We found that culture of human preimplantation embryos for 6 days resulted in medium-dependent changes in expression level of genes involved in apoptosis, protein degradation, metabolism and cell cycle regulation. Several biological pathways, including pathways involved in proliferation, were upregulated in Vitrolife G5 medium compared with HTF medium. These results correlate with the findings that embryos in the G5 group consisted of more cells on Day 2 and Day 3 and might be related to altered birth weight.

Clear differences in composition between Vitrolife $\mathrm{G} 5$ and HTF are the albumin source and the addition of amino acids, vitamins, hyaluronan and lipoic acid in G5. Little is known about the effects of specific components on the gene expression in embryos. However, the expression of numerous genes in different mammalian cell lines are regulated by the cellular concentration of amino acids (Jousse et al., 2004). Culture of mouse embryos in medium which lacks amino acids (Whitten's medium) affected biological and molecular processes involved in ribosome biogenesis and protein synthesis, cell organization and biogenesis, apoptosis, regulation of cell cycle and proliferation, and selenium binding when compared with embryos cultured in medium containing amino acids (K-modified simplex optimized medium + amino acids) and in vivo counterparts (Rinaudo and Schultz, 2004). In our study we also compared a medium without (HTF) and with (Vitrolife G5) amino acids and found differences in similar processes. 


\section{Other ART-procedure related factors}

Although subfertility itself plays a significant role in the adverse perinatal outcome, it has been found that even in the same mother an ART singleton has a poorer outcome than its non-ART sibling, indicating a risk attributable to ART (Pinborg et al., 2013). In this thesis we focused on the effect of embryo culture medium on the health outcome of IVF children. However, next to embryo culture media there are also other ART-procedure related factors that are at least partially responsible for the adverse outcome in IVF children. For example, the method of fertilization is such a factor, as a lower risk of preterm birth or low birth weight is seen in ICSI as compared to IVF singletons (Pinborg et al., 2013). However, this finding can be confounded by the fact that in ICSI the majority of the female partners are reproductively healthy. The transfer of frozen/thawed embryos is another factor that affects perinatal outcome. Although singletons born after frozen embryo transfer (FET) have a better perinatal outcome compared with singletons born after fresh embryo transfer regarding low birth weight and preterm birth, they seem to have an increased risk of being large for gestational age (LGA) (Pelkonen et al., 2010; Sazonova et al., 2012; Pinborg et al., 2014). The higher birth weight in FET singletons was also evident in a study in which one singleton sibling born after fresh transfer and the other after FET were compared, even after adjustment for maternal age and birth order (Henningsen et al., 2011). Another factor that might influence perinatal outcome in IVF children is the duration of culture (cleavage versus blastocyst transfers). In a large Swedish cohort study, it was shown that the risk of preterm birth among singletons was significantly greater after blastocyst transfer than after cleavage-stage transfer (Kallen et al., 2010). The study included an unknown proportion of FET cycles, which can confound the outcome. However, the findings were confirmed by other large studies in which FET cycles were excluded (Kalra et al., 2012; Dar et al., 2013). Several studies found a significant effect of extended embryo culture on the proportion of LGA babies and birth weight (Makinen et al., 2013; Zhu et al., 2014b; Kaartinen et al., 2015). These studies support our findings that embryo culture media affect perinatal outcome, however, the effects of extended culture might also be associated with other culture conditions such as $\mathrm{pH}$, mineral oil and oxygen level. The controlled ovarian stimulation (COS) regimens in ART also contribute to the adverse perinatal outcome in ART children. IVF singletons have a worse outcome than singletons after ovulation induction with IUI, which may be associated with the more aggressive ovarian stimulation or with a negative impact of the IVF laboratory procedures (Wang et al., 2002; De Sutter et al., 2005).

It is important to note that there are difficulties with the analysis in human studies. First, humans vary greatly in their genetic background when compared with genetically identical animal models, which makes it more difficult to detect 
effects in humans. Second, the possible association between different ARTprocedure related factors, such as FET cycles and COS, makes it difficult to isolate the contribution of specific ART-procedure related factors to the overall risk of an adverse perinatal outcome after ART. However, our studies together with other studies have shown that ART-procedure related factors - next to subfertility itself play an important role in the adverse perinatal outcome in ART singletons. The poorer outcome in ART singletons are caused by multiple factors with culture medium being at least one of the factors responsible.

\section{Wider implications}

The focus of attention in most studies on the perinatal outcome in ART children, including ours, is directed towards preterm birth and birth weight. The clinical relevance of the differences in birth weight and postnatal weight that we have found in our studies should be established in future studies. The extreme differences in birth weight that have been observed in animal studies, such as the large offspring syndrome, have not been observed in humans. However, according to the DOHaD hypothesis, exposure to a suboptimal environment during prenatal life can predispose the individual to chronic diseases later in life (Barker, 1997). It is thought that this is driven by epigenetic changes in the gametes, embryo and fetus via responses to environmental cues to optimize future development (Fleming et al., 2015). As described in the Introduction, in utero exposure to a period of famine has important effects on health in later life. It has been found that this exposure was associated with epigenetic differences in exposed individuals 60 years later (Heijmans et al., 2008). Evidence of embryo and periconceptional programming also comes from the animal studies in which the effect of the maternal periconceptional nutritional status has been evaluated (Fleming et al., 2015). For example, maternal low protein diet fed exclusively during the preimplantation period resulted in a gender-specific altered gene expression in rat fetuses (Kwong et al., 2007).

Regarding the still young age of human ART progeny, it is unknown what the longterm health effects will be. However, there are some similarities between the adverse cardiometabolic health outcomes identified in the studies from periconceptional and embryo exposures to poor maternal nutrition and that in ART children. Children born after ART are more likely to have elevated systolic and diastolic blood pressures, higher fasting glucose levels, vascular dysfunction and increased central adiposity (Ceelen et al., 2008; Sakka et al., 2010; Belva et al., 2012; Scherrer et al., 2012; Valenzuela-Alcaraz et al., 2013; Seggers et al., 2014; Pontesilli et al., 2015). Taken together, this may imply a serious health risk for ART offspring worldwide. 


\section{Conclusion and future prospects}

ART children may be predisposed for adult health risks via subtle epigenetic changes. With the growing number of children conceived after ART, it is important that the safety of ART is investigated. The development of embryo culture media has been driven by promoting embryo growth and improving pregnancy rates, however, full knowledge about the requirements of human embryos is lacking. Future embryo culture medium research should also focus on perinatal/postnatal outcome and infant, adolescent and adult health in ART progeny.

Currently, a list of the full composition of embryo culture media is available for only some culture media. Like drug companies, companies that produce embryo culture media should make the full composition of embryo culture media publicly available to enable comparison of culture media. There are limited constraints on introducing new culture media, even if they contain new growth factors of unknown effect on pregnancy rates, let alone the health of the children born. Introduction of new culture media or the addition of new supplements should be based on properly conducted RCTs. However, the need to consider the safety of future changes to culture media composition and laboratory protocols before introducing them into the clinical setting is also a responsibility of the clinical embryologists. They can also play an important role in delivering information on the effects of those media already introduced. Furthermore, long-term follow-up procedures should be an integral part of the treatment protocol in every IVF center worldwide. Currently, in our center we are involved in a study that investigates the effect of culture medium on the health of IVF children at the age of 9 years, with the focus on physiological (blood pressure and endothelial function), metabolic (lipid profile and glucose), and anthropometric (length, weight and skin fold thickness) examinations. Recently, also a new RCT started to compare pregnancy and perinatal outcome between the sequential culture medium Vitrolife G5 and Irvine's single step Continuous Single Culture medium. ART clinicians and embryologists should provide robust scientific evidence for new procedures they introduce in their clinics and labs. This evidence should include side effects and long-term health consequences for mother and child. 
References 


\section{References}

Alpha Scientists in Reproductive Medicine and ESHRE Special Interest Group of Embryology. The Istanbul consensus workshop on embryo assessment: proceedings of an expert meeting. Hum Reprod 2011; 26:1270-1283.

Arias ME, Ross PJ, Felmer RN. Culture medium composition affects the gene expression pattern and in vitro development potential of bovine somatic cell nuclear transfer (SCNT) embryos. Biol Res 2013; 46:452-462.

Assou S, Boumela I, Haouzi D, Anahory T, Dechaud H, De Vos J, Hamamah S. Dynamic changes in gene expression during human early embryo development: from fundamental aspects to clinical applications. Hum Reprod Update 2011; 17:272-290.

Balaban B, Urman B. Comparison of two sequential media for culturing cleavage-stage embryos and blastocysts: embryo characteristics and clinical outcome. Reprod Biomed Online 2005; 10:485-491.

Baltz JM. Media composition: salts and osmolality. Methods Mol Biol 2012; 912:61-80.

Barker DJ. Maternal nutrition, fetal nutrition, and disease in later life. Nutrition 1997; 13:807-813.

Basatemur E, Shevlin M, Sutcliffe A. Growth of children conceived by IVF and ICSI up to 12years of age. Reprod Biomed Online 2010; 20:144-149.

Bavister BD. Culture of preimplantation embryos: facts and artifacts. Hum Reprod Update 1995; 1:91-148.

Belva F, Painter R, Bonduelle M, Roelants M, Devroey P, De Schepper J. Are ICSI adolescents at risk for increased adiposity? Hum Reprod 2012; 27:257-264.

Berkey CS, Reed RB. A model for describing normal and abnormal growth in early childhood. Hum Biol 1987; 59:973-987.

Beydoun HA, Sicignano N, Beydoun MA, Matson DO, Bocca S, Stadtmauer L, Oehninger S. A crosssectional evaluation of the first cohort of young adults conceived by in vitro fertilization in the United States. Fertil Steril 2010; 94:2043-2049.

Biggers JD, McGinnis LK, Lawitts JA. Enhanced effect of glycyl-L-glutamine on mouse preimplantation embryos in vitro. Reprod Biomed Online 2004a; 9:59-69.

Biggers JD, McGinnis LK, Summers MC. Discrepancies between the effects of glutamine in cultures of preimplantation mouse embryos. Reprod Biomed Online 2004b; 9:70-73.

Biggers JD, Summers MC. Choosing a culture medium: making informed choices. Fertil Steril 2008; 90:473-483.

Bisioli C, Valcarcel A, Tiveron M, Quintana R, Young E, Sueldo C. Better embryo quality and reproductive performance using sequential media in day 3 transfers. Fertil Steril 2003; 80:S57-S57.

Blair PS, Drewett RF, Emmett PM, Ness A, Emond AM. Family, socioeconomic and prenatal factors associated with failure to thrive in the Avon Longitudinal Study of Parents and Children (ALSPAC). Int J Epidemiol 2004; 33:839-847.

Blickstein I, Kalish RB. Birth weight discordance in multiple pregnancy. Twin Res 2003; 6:526-531.

Bloise E, Feuer SK, Rinaudo PF. Comparative intrauterine development and placental function of ART concepti: implications for human reproductive medicine and animal breeding. Hum Reprod Update 2014; 20:822-839.

Boivin J, Bunting L, Collins JA, Nygren KG. International estimates of infertility prevalence and treatment-seeking: potential need and demand for infertility medical care. Hum Reprod 2007; 22:1506-1512.

Bonduelle M, Liebaers I, Deketelaere V, Derde MP, Camus M, Devroey P, Van Steirteghem A. Neonatal data on a cohort of 2889 infants born after ICSI (1991-1999) and of 2995 infants born after IVF (1983-1999). Hum Reprod 2002; 17:671-694. 
Bontekoe S, Heineman MJ, Johnson N, Blake D. Adherence compounds in embryo transfer media for assisted reproductive technologies. Cochrane Database Syst Rev 2014; 2:CD007421.

Bouillon C, Leandri R, Desch L, Ernst A, Bruno C, Cerf C, Chiron A, Souchay C, Burguet A, Jimenez C et al. Does Embryo Culture Medium Influence the Health and Development of Children Born after In Vitro Fertilization? PloS one 2016; 11:e0150857.

Bowman P, McLaren A. Viability and growth of mouse embryos after in vitro culture and fusion. $J$ Embryol Exp Morphol 1970; 23:693-704.

Braakhekke M, Kamphuis EI, Dancet EA, Mol F, van der Veen F, Mol BW. Ongoing pregnancy qualifies best as the primary outcome measure of choice in trials in reproductive medicine: an opinion paper. Fertil Steril 2014; 101:1203-1204.

Bridges PJ, Jeoung M, Kim H, Kim JH, Lee DR, Ko C, Baker DJ. Methodology matters: IVF versus ICSI and embryonic gene expression. Reprod Biomed Online 2011; 23:234-244.

Brinster RL. A Method for in Vitro Cultivation of Mouse Ova from Two-Cell to Blastocyst. Exp Cell Res 1963; 32:205-208.

Cagnone GL, Dufort I, Vigneault C, Sirard MA. Differential gene expression profile in bovine blastocysts resulting from hyperglycemia exposure during early cleavage stages. Biol Reprod 2012; 86:50.

Carone BR, Fauquier L, Habib N, Shea JM, Hart CE, Li R, Bock C, Li C, Gu H, Zamore PD et al. Paternally induced transgenerational environmental reprogramming of metabolic gene expression in mammals. Cell 2010; 143:1084-1096.

Carrasco B, Boada M, Rodriguez I, Coroleu B, Barri PN, Veiga A. Does culture medium influence offspring birth weight? Fertil Steril 2013; 100:1283-1288.

Ceelen M, van Weissenbruch MM, Prein J, Smit JJ, Vermeiden JP, Spreeuwenberg M, van Leeuwen FE, Delemarre-van de Waal HA. Growth during infancy and early childhood in relation to blood pressure and body fat measures at age 8-18 years of IVF children and spontaneously conceived controls born to subfertile parents. Hum Reprod 2009; 24:2788-2795.

Ceelen M, van Weissenbruch MM, Vermeiden JP, van Leeuwen FE, Delemarre-van de Waal HA. Cardiometabolic differences in children born after in vitro fertilization: follow-up study. $J$ Clin Endocrinol Metab 2008; 93:1682-1688.

Centers for Disease Control and Prevention, American Society for Reproductive Medicine, Society for Assisted Reproductive Technology. 2010 Assisted Reproductive Technology National Summary Report. Atlanta: U.S. Department of Health and Human Services, 2012.

Centers for Disease Control and Prevention, American Society for Reproductive Medicine, Society for Assisted Reproductive Technology. 2011 Assisted Reproductive Technology National Summary Report. Atlanta (GA): U.S. Department of Health and Human Services, 2013.

Chan B, Dodsworth N, Woodrow J, Tucker A, Harris R. Site-specific N-terminal auto-degradation of human serum albumin. Eur J Biochem 1995; 227:524-528.

Chatot CL, Ziomek CA, Bavister BD, Lewis JL, Torres I. An improved culture medium supports development of random-bred 1-cell mouse embryos in vitro. J Reprod Fertil 1989; 86:679688.

Choi SJ, Kim SK, Chun KW, Koong MK, Kang IS, Yang KM, Byun HK. Efficacy of sequential culture media system in the frozen-thawed embryo transfer of human pronuclear zygotes. Hum Reprod 2004; 19 Suppl 1:i108.

Chronopoulou E, Harper JC. IVF culture media: past, present and future. Hum Reprod Update 2015; 21:39-55.

Conaghan J. Time-lapse imaging of preimplantation embryos. Semin Reprod Med 2014; 32:134-140.

Cossiello RD, Aggelis A, Faundes D, Petta CA. Morphological differences in human zygotes and embryos cultured in different media. Zygote 2012; 20:399-405. 
Dar S, Librach CL, Gunby J, Bissonnette F, Cowan L, Fertility IVFDGoC, Andrology S. Increased risk of preterm birth in singleton pregnancies after blastocyst versus Day 3 embryo transfer: Canadian ART Register (CARTR) analysis. Hum Reprod 2013; 28:924-928.

De Silva M. Effect of storage of Ham's F-10 medium on one-cell mouse embryo development in vitro. J Assist Reprod Genet 1993; 10:238-241.

De Sutter P, Delbaere I, Gerris J, Verstraelen H, Goetgeluk S, Van der Elst J, Temmerman M, Dhont $M$. Birth weight of singletons after assisted reproduction is higher after single- than after double-embryo transfer. Hum Reprod 2006; 21:2633-2637.

De Sutter P, Veldeman L, Kok P, Szymczak N, Van der Elst J, Dhont M. Comparison of outcome of pregnancy after intra-uterine insemination (IUI) and IVF. Hum Reprod 2005; 20:1642-1646.

De Vos A, Janssens R, Van de Velde H, Haentjens P, Bonduelle M, Tournaye H, Verheyen G. The type of culture medium and the duration of in vitro culture do not influence birth weight of ART singletons. Hum Reprod 2015; 30:20-27.

Delle Piane L, Lin W, Liu X, Donjacour A, Minasi P, Revelli A, Maltepe E, Rinaudo PF. Effect of the method of conception and embryo transfer procedure on mid-gestation placenta and fetal development in an IVF mouse model. Hum Reprod 2010; 25:2039-2046.

Devreker F, Winston RM, Hardy K. Glutamine improves human preimplantation development in vitro. Fertil Steril 1998; 69:293-299.

Doherty AS, Mann MR, Tremblay KD, Bartolomei MS, Schultz RM. Differential effects of culture on imprinted $\mathrm{H} 19$ expression in the preimplantation mouse embryo. Biol Reprod 2000; 62:1526-1535.

Dondorp W, de Wert G. Innovative reproductive technologies: risks and responsibilities. Hum Reprod 2011; 26:1604-1608.

Donjacour A, Liu X, Lin W, Simbulan R, Rinaudo PF. In vitro fertilization affects growth and glucose metabolism in a sex-specific manner in an outbred mouse model. Biol Reprod 2014; 90:80.

Dumoulin JC, Land JA, Van Montfoort AP, Nelissen EC, Coonen E, Derhaag JG, Schreurs IL, Dunselman GA, Kester AD, Geraedts JP et al. Effect of in vitro culture of human embryos on birth weight of newborns. Hum Reprod 2010; 25:605-612.

Duranthon V, Watson AJ, Lonergan P. Preimplantation embryo programming: transcription, epigenetics, and culture environment. Reproduction 2008; 135:141-150.

Dyrlund TF, Kirkegaard K, Poulsen ET, Sanggaard KW, Hindkjaer JJ, Kjems J, Enghild JJ, Ingerslev HJ. Unconditioned commercial embryo culture media contain a large variety of non-declared proteins: a comprehensive proteomics analysis. Hum Reprod 2014; 29:2421-2430.

Eaton JL, Lieberman ES, Stearns C, Chinchilla M, Racowsky C. Embryo culture media and neonatal birth weight following IVF. Hum Reprod 2012; 27:375-379.

Ecker DJ, Stein P, Xu Z, Williams CJ, Kopf GS, Bilker WB, Abel T, Schultz RM. Long-term effects of culture of preimplantation mouse embryos on behavior. Proc Natl Acad Sci U S A 2004; 101:1595-1600.

Erbach GT, Lawitts JA, Papaioannou VE, Biggers JD. Differential growth of the mouse preimplantation embryo in chemically defined media. Biol Reprod 1994; 50:1027-1033.

ESHRE, European Society of Human Reproduction and Embryology. 2014. ART fact sheet (July 2014): http://www.eshre.eu/Guidelines-and-Legal/ART-fact-sheet.aspx

Eskild A, Monkerud L, Tanbo T. Birth weight and placental weight; do changes in culture media used for IVF matter? Comparisons with spontaneous pregnancies in the corresponding time periods. Hum Reprod 2013; 28:3207-3214.

Evers JL. Female subfertility. Lancet 2002; 360:151-159.

Fang S, Weissman AM. A field guide to ubiquitylation. Cell Mol Life Sci 2004; 61:1546-1561.

Fauser BC, Devroey P, Diedrich K, Balaban B, Bonduelle M, Delemarre-van de Waal HA, Estella C, Ezcurra D, Geraedts JP, Howles CM et al. Health outcomes of children born after IVF/ICSI: a review of current expert opinion and literature. Reprod Biomed Online 2014; 28:162-182. 
Fernandez-Gonzalez R, Moreira P, Bilbao A, Jimenez A, Perez-Crespo M, Ramirez MA, Rodriguez De Fonseca F, Pintado B, Gutierrez-Adan A. Long-term effect of in vitro culture of mouse embryos with serum on mRNA expression of imprinting genes, development, and behavior. Proc Natl Acad Sci U S A 2004; 101:5880-5885.

Ferraretti AP, Goossens V, Kupka M, Bhattacharya S, de Mouzon J, Castilla JA, Erb K, Korsak V, Nyboe Andersen A, European IVFMCftESoHR et al. Assisted reproductive technology in Europe, 2009: results generated from European registers by ESHRE. Hum Reprod 2013; 28:23182331.

Fleming TP, Velazquez MA, Eckert JJ. Embryos, DOHaD and David Barker. J Dev Orig Health Dis 2015; 6:377-383.

Fleming TP, Velazquez MA, Eckert JJ, Lucas ES, Watkins AJ. Nutrition of females during the periconceptional period and effects on foetal programming and health of offspring. Anim Reprod Sci 2012; 130:193-197.

Flicek P, Ahmed I, Amode MR, Barrell D, Beal K, Brent S, Carvalho-Silva D, Clapham P, Coates G, Fairley S et al. Ensembl 2013. Nucleic Acids Res 2013; 41:D48-55.

Gardner DK. Changes in requirements and utilization of nutrients during mammalian preimplantation embryo development and their significance in embryo culture. Theriogenology 1998; 49:83-102.

Gardner DK. In vitro fertilization: a practical approach, 2007. Informa Healthcare.

Gardner DK, Hamilton R, McCallie B, Schoolcraft WB, Katz-Jaffe MG. Human and mouse embryonic development, metabolism and gene expression are altered by an ammonium gradient in vitro. Reproduction 2013; 146:49-61.

Gardner DK, Lane M. Amino acids and ammonium regulate mouse embryo development in culture. Biol Reprod 1993; 48:377-385.

Gardner DK, Lane M. Culture and selection of viable blastocysts: a feasible proposition for human IVF? Hum Reprod Update 1997; 3:367-382.

Gardner DK, Lane M. Culture of the mammalian preimplantation embryo. 1st edn, 2004. Oxford University Press, Oxford, UK.

Gardner DK, Lane M. Ex vivo early embryo development and effects on gene expression and imprinting. Reprod Fertil Dev 2005; 17:361-370.

Gardner DK, Lane M, Stevens J, Schoolcraft WB. Noninvasive assessment of human embryo nutrient consumption as a measure of developmental potential. Fertil Steril 2001; 76:1175-1180.

Gardner DK, Pool TB, Lane M. Embryo nutrition and energy metabolism and its relationship to embryo growth, differentiation, and viability. Semin Reprod Med 2000; 18:205-218.

Gardner DK, Surrey E, Minjarez D, Leitz A, Stevens J, Schoolcraft WB. Single blastocyst transfer: a prospective randomized trial. Fertil Steril 2004; 81:551-555.

Gentleman RC, Carey VJ, Bates DM, Bolstad B, Dettling M, Dudoit S, Ellis B, Gautier L, Ge Y, Gentry J et al. Bioconductor: open software development for computational biology and bioinformatics. Genome Biol 2004; 5:R80.

Gilbert R, Hwan S, Vermilyea M, Graham J, Tucker M. Low levels of ammonium detected in spent media from mouse or human embryos cultured in continuous single culture ${ }^{\mathrm{TM}}$ (CSC) medium are not detrimental to development. Fertil Steril 2012; 98:S169.

Giritharan G, Li MW, Di Sebastiano F, Esteban FJ, Horcajadas JA, Lloyd KC, Donjacour A, Maltepe E, Rinaudo PF. Effect of ICSI on gene expression and development of mouse preimplantation embryos. Hum Reprod 2010; 25:3012-3024.

Gray CW, Morgan PM, Kane MT. Purification of an embryotrophic factor from commercial bovine serum albumin and its identification as citrate. J Reprod Fertil 1992; 94:471-480.

Griffiths LJ, Dezateux C, Cole TJ. Differential parental weight and height contributions to offspring birth weight and weight gain in infancy. Int J Epidemiol 2007; 36:104-107. 
Grondahl ML, Yding Andersen C, Bogstad J, Nielsen FC, Meinertz H, Borup R. Gene expression profiles of single human mature oocytes in relation to age. Hum Reprod 2010; 25:957-968.

Hadlock FP, Harrist RB, Sharman RS, Deter RL, Park SK. Estimation of fetal weight with the use of head, body, and femur measurements--a prospective study. Am J Obstet Gynecol 1985; 151:333-337.

Hadlock FP, Shah YP, Kanon DJ, Lindsey JV. Fetal crown-rump length: reevaluation of relation to menstrual age (5-18 weeks) with high-resolution real-time US. Radiology 1992; 182:501505.

Hammon DS, Wang S, Holyoak GR. Effects of ammonia during different stages of culture on development of in vitro produced bovine embryos. Anim Reprod Sci 2000; 59:23-30.

Hardarson T, Bungum M, Conaghan J, Meintjes M, Chantilis SJ, Molnar L, Gunnarsson K, Wikland M. Noninferiority, randomized, controlled trial comparing embryo development using media developed for sequential or undisturbed culture in a time-lapse setup. Fertil Steril 2015; 104:1452-1459 e1454.

Hart R, Norman RJ. The longer-term health outcomes for children born as a result of IVF treatment: Part I--General health outcomes. Hum Reprod Update 2013a; 19:232-243.

Hart R, Norman RJ. The longer-term health outcomes for children born as a result of IVF treatment: Part II--Mental health and development outcomes. Hum Reprod Update 2013b; 19:244250.

Hashimoto S, Nishihara T, Murata Y, Oku H, Nakaoka Y, Fukuda A, Morimoto Y. Medium without ammonium accumulation supports the developmental competence of human embryos. $J$ Reprod Dev 2008; 54:370-374.

Hassani F, Eftekhari-Yazdi P, Karimian L, Rezazadeh Valojerdi M, Movaghar B, Fazel M, Fouladi HR, Shabani F, Johansson L. The Effects of ISM1 Medium on Embryo Quality and Outcomes of IVF/ICSI Cycles. Int J Fertil Steril 2013; 7:108-115.

Hauspie RC, Molinari L. Parametric models for postnatal growth. In Hauspie RC, Cameron N and Molinari L (eds) Methods in Human Growth Research. 2004. Cambridge University Press, New York, pp. 205-233.

Heijmans BT, Tobi EW, Stein AD, Putter H, Blauw GJ, Susser ES, Slagboom PE, Lumey LH. Persistent epigenetic differences associated with prenatal exposure to famine in humans. Proc Natl Acad Sci U S A 2008; 105:17046-17049.

Helmerhorst FM, Perquin DA, Donker D, Keirse MJ. Perinatal outcome of singletons and twins after assisted conception: a systematic review of controlled studies. BMJ 2004; 328:261.

Henningsen AK, Pinborg A. Birth and perinatal outcomes and complications for babies conceived following ART. Semin Fetal Neonatal Med 2014; 19:234-238.

Henningsen AK, Pinborg A, Lidegaard O, Vestergaard C, Forman JL, Andersen AN. Perinatal outcome of singleton siblings born after assisted reproductive technology and spontaneous conception: Danish national sibling-cohort study. Fertil Steril 2011; 95:959-963.

Hindmarsh PC, Geary MP, Rodeck CH, Kingdom JC, Cole TJ. Factors predicting ante- and postnatal growth. Pediatr Res 2008; 63:99-102.

Hoogendijk CF, Kruger TF, de Beer ML, Siebert TI, Henkel R. A study of two sequential culture media - impact on embryo quality and pregnancy rates. Sajog-S Afr J Obstet 2007; 13:52-58.

Hoopmann M, Abele H, Wagner N, Wallwiener D, Kagan KO. Performance of 36 different weight estimation formulae in fetuses with macrosomia. Fetal Diagn Ther 2010; 27:204-213.

Hossain A, Osuamkpe C, Hossain S, Phelps J. Shelf life of embryo culture media: Buffering potential of media apparently not the determining factor. Middle East Fertil Soc J 2010; 15:179-182.

Hsieh RH, Au HK, Yeh TS, Chang SJ, Cheng YF, Tzeng CR. Decreased expression of mitochondrial genes in human unfertilized oocytes and arrested embryos. Fertil Steril 2004; 81 Suppl 1:912-918. 
Huppertz B, Herrler A. Regulation of proliferation and apoptosis during development of the preimplantation embryo and the placenta. Birth Defects Res C Embryo Today 2005; 75:249-261.

Jackson RA, Gibson KA, Wu YW, Croughan MS. Perinatal outcomes in singletons following in vitro fertilization: a meta-analysis. Obstet Gynecol 2004; 103:551-563.

Jenss RM, Bayley N. A mathematical method for studying the growth of a child. Hum Biol 1937; 9:556-563.

Jousse C, Averous J, Bruhat A, Carraro V, Mordier S, Fafournoux P. Amino acids as regulators of gene expression: molecular mechanisms. Biochem Biophys Res Commun 2004; 313:447-452.

Jungheim ES, Louden ED, Chi MM, Frolova Al, Riley JK, Moley KH. Preimplantation exposure of mouse embryos to palmitic acid results in fetal growth restriction followed by catch-up growth in the offspring. Biol Reprod 2011; 85:678-683.

Kaartinen NM, Kananen KM, Rodriguez-Wallberg KA, Tomas CM, Huhtala HS, Tinkanen HI. Male gender explains increased birth weight in children born after transfer of blastocysts. Hum Reprod 2015; 30:2312-2320.

Kallen B, Finnstrom O, Lindam A, Nilsson E, Nygren KG, Olausson PO. Blastocyst versus cleavage stage transfer in in vitro fertilization: differences in neonatal outcome? Fertil Steril 2010; 94:1680-1683.

Kalra SK, Ratcliffe SJ, Barnhart KT, Coutifaris C. Extended embryo culture and an increased risk of preterm delivery. Obstet Gynecol 2012; 120:69-75.

Khosla S, Dean W, Brown D, Reik W, Feil R. Culture of preimplantation mouse embryos affects fetal development and the expression of imprinted genes. Biol Reprod 2001a; 64:918-926.

Khosla S, Dean W, Reik W, Feil R. Culture of preimplantation embryos and its long-term effects on gene expression and phenotype. Hum Reprod Update 2001b; 7:419-427.

Kleijkers SH, Eijssen LM, Coonen E, Derhaag JG, Mantikou E, Jonker MJ, Mastenbroek S, Repping S, Evers JL, Dumoulin JC et al. Differences in gene expression profiles between human preimplantation embryos cultured in two different IVF culture media. Hum Reprod 2015a; 30:2303-2311.

Kleijkers SH, van Montfoort AP, Smits L, Coonen E, Derhaag JG, Evers JL, Dumoulin JC. Age of G-1 PLUS v5 embryo culture medium is inversely associated with birth weight of the newborn. Hum Reprod 2015b; 30:1352-1357.

Kleijkers SH, van Montfoort AP, Smits L, Viechtbauer W, Roseboom TJ, Nelissen EC, Coonen E, Derhaag JG, Bastings L, Schreurs IE et al. IVF culture medium affects post-natal weight in humans during the first 2 years of life. Hum Reprod 2014; 29:661-669.

Kolesnikov N, Hastings E, Keays M, Melnichuk O, Tang YA, Williams E, Dylag M, Kurbatova N, Brandizi $\mathrm{M}$, Burdett $\mathrm{T}$ et al. ArrayExpress update--simplifying data submissions. Nucleic Acids Res 2015; 43:D1113-1116.

Kramer MS. Determinants of low birth weight: methodological assessment and meta-analysis. Bull World Health Organ 1987; 65:663-737.

Kupka M, D'Hooghe T, Ferraretti A, de Mouzon J, Erb K, Castilla J, Calhaz-Jorge C, De Geyter C, Goossens V, European IVF-Monitoring Consortium. Assisted reproductive technology in Europe, 2011: results generated from European registers by ESHRE. Hum Reprod 2016; 31:233-248.

Kupka MS, Ferraretti AP, de Mouzon J, Erb K, D'Hooghe T, Castilla JA, Calhaz-Jorge C, De Geyter C, Goossens V, European Ivf-Monitoring Consortium ftESoHR et al. Assisted reproductive technology in Europe, 2010: results generated from European registers by ESHREdagger. Hum Reprod 2014; 29:2099-2113.

Kwong WY, Miller DJ, Wilkins AP, Dear MS, Wright JN, Osmond C, Zhang J, Fleming TP. Maternal low protein diet restricted to the preimplantation period induces a gender-specific change on hepatic gene expression in rat fetuses. Mol Reprod Dev 2007; 74:48-56. 
Kwong WY, Wild AE, Roberts P, Willis AC, Fleming TP. Maternal undernutrition during the preimplantation period of rat development causes blastocyst abnormalities and programming of postnatal hypertension. Development 2000; 127:4195-4202.

Land JA, Evers JL. Risks and complications in assisted reproduction techniques: Report of an ESHRE consensus meeting. Hum Reprod 2003; 18:455-457.

Lane M, Gardner DK. Increase in postimplantation development of cultured mouse embryos by amino acids and induction of fetal retardation and exencephaly by ammonium ions. $J$ Reprod Fertil 1994; 102:305-312.

Lane M, Gardner DK. Amino acids and vitamins prevent culture-induced metabolic perturbations and associated loss of viability of mouse blastocysts. Hum Reprod 1998; 13:991-997.

Lane M, Gardner DK. Ammonium induces aberrant blastocyst differentiation, metabolism, pH regulation, gene expression and subsequently alters fetal development in the mouse. Biol Reprod 2003; 69:1109-1117.

Lane M, Gardner DK. Embryo culture medium: which is the best? Best Pract Res Clin Obstet Gynaecol 2007; 21:83-100.

Lawitts J, Biggers J. Optimization of mouse embryo culture media using simplex methods. J Reprod Fertil 1991; 91:543-556.

Le F, Wang LY, Wang N, Li L, Li le J, Zheng YM, Lou HY, Liu XZ, Xu XR, Sheng JZ et al. In vitro fertilization alters growth and expression of Igf2/H19 and their epigenetic mechanisms in the liver and skeletal muscle of newborn and elder mice. Biol Reprod 2013; 88:75.

Lee SH, Lee MY, Chiang TL, Lee MS, Lee MC. Child growth from birth to 18 months old born after assisted reproductive technology--results of a national birth cohort study. Int J Nurs Stud 2010; 47:1159-1166.

Lemmen JG, Pinborg A, Rasmussen S, Ziebe S. Birth weight distribution in ART singletons resulting from embryo culture in two different culture media compared with the national population. Hum Reprod 2014; 29:2326-2332.

Li Q, Wang WJ, Zhang NF, Ouyang NY, Li RQ, Mai MQ, Lao WS, Yang DZ, Zhang QX. Ammonium concentration of spent medium provides a noninvasive assessment of embryonic developmental potential in IVF. Reprod Sci 2013; 20:1316-1320.

Lin S, Li M, Lian Y, Chen L, Liu P. No effect of embryo culture media on birth weight and length of newborns. Hum Reprod 2013; 28:1762-1767.

Liu Z, Foote RH. Effects of amino acids on the development of in-vitro matured/in-vitro fertilization bovine embryos in a simple protein-free medium. Hum Reprod 1995; 10:2985-2991.

Lucas E. Epigenetic effects on the embryo as a result of periconceptional environment and assisted reproduction technology. Reprod Biomed Online 2013; 27:477-485.

MacLaughlin SM, Walker SK, Roberts CT, Kleemann DO, McMillen IC. Periconceptional nutrition and the relationship between maternal body weight changes in the periconceptional period and feto-placental growth in the sheep. J Physiol 2005; 565:111-124.

Makinen S, Soderstrom-Anttila V, Vainio J, Suikkari AM, Tuuri T. Does long in vitro culture promote large for gestational age babies? Hum Reprod 2013; 28:828-834.

Mann MR, Lee SS, Doherty AS, Verona RI, Nolen LD, Schultz RM, Bartolomei MS. Selective loss of imprinting in the placenta following preimplantation development in culture. Development 2004; 131:3727-3735.

Mantikou E, Jonker MJ, de Jong M, Wong KM, van Montfoort APA, Breit TM, Repping S, Mastenbroek S. Factors affecting the transcriptome of human preimplantation embryos. Hum Reprod 2013a; 28 Suppl 1:i202-i202.

Mantikou E, Jonker MJ, Wong KM, van Montfoort AP, de Jong M, Breit TM, Repping S, Mastenbroek $S$. Factors affecting the gene expression of in vitro cultured human preimplantation embryos. Hum Reprod 2016; 31:298-311. 
Mantikou E, Youssef MA, van Wely M, van der Veen F, Al-Inany HG, Repping S, Mastenbroek S. Embryo culture media and IVF/ICSI success rates: a systematic review. Hum Reprod Update 2013b; 19:210-220.

Market-Velker BA, Fernandes AD, Mann MR. Side-by-side comparison of five commercial media systems in a mouse model: suboptimal in vitro culture interferes with imprint maintenance. Biol Reprod 2010; 83:938-950.

McDonald SD, Han Z, Mulla S, Murphy KE, Beyene J, Ohlsson A, Knowledge Synthesis G. Preterm birth and low birth weight among in vitro fertilization singletons: a systematic review and meta-analyses. Eur J Obstet Gynecol Reprod Biol 2009; 146:138-148.

McDonald SD, Han Z, Mulla S, Ohlsson A, Beyene J, Murphy KE, Knowledge Synthesis G. Preterm birth and low birth weight among in vitro fertilization twins: a systematic review and metaanalyses. Eur J Obstet Gynecol Reprod Biol 2010; 148:105-113.

Meintjes M. Media composition: macromolecules and embryo growth. Methods Mol Biol 2012; 912:107-127.

Meintjes M, Chantilis SJ, Ward DC, Douglas JD, Rodriguez AJ, Guerami AR, Bookout DM, Barnett BD, Madden JD. A randomized controlled study of human serum albumin and serum substitute supplement as protein supplements for IVF culture and the effect on live birth rates. Hum Reprod 2009; 24:782-789.

Mesman I, Roseboom TJ, Bonsel GJ, Gemke RJ, van der Wal MF, Vrijkotte TG. Maternal prepregnancy body mass index explains infant's weight and BMI at 14 months: results from a multi-ethnic birth cohort study. Arch Dis Child 2009; 94:587-595.

Moravek M, Fisseha S, Swain JE. Dipeptide forms of glycine support mouse preimplantation embryo development in vitro and provide protection against high media osmolality. J Assist Reprod Genet 2012; 29:283-290.

Morbeck DE, Krisher RL, Herrick JR, Baumann NA, Matern D, Moyer T. Composition of commercial media used for human embryo culture. Fertil Steril 2014a; 102:759-766 e759.

Morbeck DE, Paczkowski M, Fredrickson JR, Krisher RL, Hoff HS, Baumann NA, Moyer T, Matern D. Composition of protein supplements used for human embryo culture. J Assist Reprod Genet 2014b; 31:1703-1711.

Mul T, Mongelli M, Gardosi J. A comparative analysis of second-trimester ultrasound dating formulae in pregnancies conceived with artificial reproductive techniques. Ultrasound Obstet Gynecol 1996; 8:397-402.

Naz RK, Janousek JT, Moody T, Stillman RJ. Factors influencing murine embryo bioassay: effects of proteins, aging of medium, and surgical glove coatings. Fertil Steril 1986; 46:914-919.

Nelissen EC, Van Montfoort AP, Coonen E, Derhaag JG, Geraedts JP, Smits LJ, Land JA, Evers JL, Dumoulin JC. Further evidence that culture media affect perinatal outcome: findings after transfer of fresh and cryopreserved embryos. Hum Reprod 2012; 27:1966-1976.

Nelissen EC, Van Montfoort AP, Smits L, Menheere PP, Evers JL, Coonen E, Derhaag JG, Peeters LL, Coumans AB, Dumoulin JC. IVF culture medium affects human intrauterine growth as early as the second trimester of pregnancy. Hum Reprod 2013; 28:2067-2074.

NVOG, Nederlandse Vereniging voor Obstetrie en Gynaecologie. IVF-resultaten: http://www.nvog.nl/voorlichting/IVF-resultaten/default.aspx

Oken E, Kleinman KP, Rich-Edwards J, Gillman MW. A nearly continuous measure of birth weight for gestational age using a United States national reference. BMC Pediatr 2003; 3:6.

Pandey S, Shetty A, Hamilton M, Bhattacharya S, Maheshwari A. Obstetric and perinatal outcomes in singleton pregnancies resulting from IVF/ICSI: a systematic review and meta-analysis. Hum Reprod Update 2012; 18:485-503.

Pelkonen S, Koivunen R, Gissler M, Nuojua-Huttunen S, Suikkari AM, Hyden-Granskog C, Martikainen $H$, Tiitinen A, Hartikainen AL. Perinatal outcome of children born after frozen and fresh embryo transfer: the Finnish cohort study 1995-2006. Hum Reprod 2010; 25:914-923. 
Pinborg A, Henningsen AA, Loft A, Malchau SS, Forman J, Andersen AN. Large baby syndrome in singletons born after frozen embryo transfer (FET): is it due to maternal factors or the cryotechnique? Hum Reprod 2014; 29:618-627.

Pinborg A, Loft A, Aaris Henningsen AK, Rasmussen S, Andersen AN. Infant outcome of 957 singletons born after frozen embryo replacement: the Danish National Cohort Study 19952006. Fertil Steril 2010; 94:1320-1327.

Pinborg A, Wennerholm UB, Romundstad LB, Loft A, Aittomaki K, Soderstrom-Anttila V, Nygren KG, Hazekamp J, Bergh C. Why do singletons conceived after assisted reproduction technology have adverse perinatal outcome? Systematic review and meta-analysis. Hum Reprod Update 2013; 19:87-104.

Pinheiro JC, Bates DM. Mixed-effects models in S and S-PLUS, 2000. Springer, New York.

Pontesilli M, Painter RC, Grooten IJ, van der Post JA, Mol BW, Vrijkotte TG, Repping S, Roseboom TJ. Subfertility and assisted reproduction techniques are associated with poorer cardiometabolic profiles in childhood. Reprod Biomed Online 2015; 30:258-267.

Quinn P. Media used in the assisted reproductive technologies laboratories. A Color Atlas for Human Assisted Reproduction: Laboratory and Clinical Insights 2003:241.

Quinn P, Kerin JF, Warnes GM. Improved pregnancy rate in human in vitro fertilization with the use of a medium based on the composition of human tubal fluid. Fertil Steril 1985; 44:493-498.

Reik W, Dean W, Walter J. Epigenetic reprogramming in mammalian development. Science 2001; 293:1089-1093.

Rimm AA, Katayama AC, Diaz M, Katayama KP. A meta-analysis of controlled studies comparing major malformation rates in IVF and ICSI infants with naturally conceived children. J Assist Reprod Genet 2004; 21:437-443.

Rinaudo P, Schultz RM. Effects of embryo culture on global pattern of gene expression in preimplantation mouse embryos. Reproduction 2004; 128:301-311.

Rinaudo PF, Giritharan G, Talbi S, Dobson AT, Schultz RM. Effects of oxygen tension on gene expression in preimplantation mouse embryos. Fertil Steril 2006; 86:1252-1265, 1265 e1251-1236.

Rooke JA, McEvoy TG, Ashworth CJ, Robinson JJ, Wilmut I, Young LE, Sinclair KD. Ovine fetal development is more sensitive to perturbation by the presence of serum in embryo culture before rather than after compaction. Theriogenology 2007; 67:639-647.

Roseboom T, de Rooij S, Painter R. The Dutch famine and its long-term consequences for adult health. Early Hum Dev 2006; 82:485-491.

Roseboom TJ, Painter RC, van Abeelen AF, Veenendaal MV, de Rooij SR. Hungry in the womb: what are the consequences? Lessons from the Dutch famine. Maturitas 2011; 70:141-145.

Saadeldin IM, Kim B, Lee B, Jang G. Effect of different culture media on the temporal gene expression in the bovine developing embryos. Theriogenology 2011; 75:995-1004.

Sakka SD, Loutradis D, Kanaka-Gantenbein C, Margeli A, Papastamataki M, Papassotiriou I, Chrousos GP. Absence of insulin resistance and low-grade inflammation despite early metabolic syndrome manifestations in children born after in vitro fertilization. Fertil Steril 2010; 94:1693-1699.

Saltvedt S, Almstrom H, Kublickas M, Reilly M, Valentin L, Grunewald C. Ultrasound dating at 12-14 or 15-20 weeks of gestation? A prospective cross-validation of established dating formulae in a population of in-vitro fertilized pregnancies randomized to early or late dating scan. Ultrasound Obstet Gynecol 2004; 24:42-50.

Sazonova A, Kallen K, Thurin-Kjellberg A, Wennerholm UB, Bergh C. Obstetric outcome in singletons after in vitro fertilization with cryopreserved/thawed embryos. Hum Reprod 2012; 27:1343-1350. 
Scherrer U, Rimoldi SF, Rexhaj E, Stuber T, Duplain H, Garcin S, de Marchi SF, Nicod P, Germond M, Allemann $Y$ et al. Systemic and pulmonary vascular dysfunction in children conceived by assisted reproductive technologies. Circulation 2012; 125:1890-1896.

Schwarzer C, Esteves TC, Arauzo-Bravo MJ, Le Gac S, Nordhoff V, Schlatt S, Boiani M. ART culture conditions change the probability of mouse embryo gestation through defined cellular and molecular responses. Hum Reprod 2012; 27:2627-2640.

Scott KA, Yamazaki Y, Yamamoto M, Lin Y, Melhorn SJ, Krause EG, Woods SC, Yanagimachi R, Sakai RR, Tamashiro KL. Glucose parameters are altered in mouse offspring produced by assisted reproductive technologies and somatic cell nuclear transfer. Biol Reprod 2010; 83:220-227.

Seggers J, Haadsma ML, La Bastide-Van Gemert S, Heineman MJ, Middelburg KJ, Roseboom TJ, Schendelaar P, Van den Heuvel ER, Hadders-Algra M. Is ovarian hyperstimulation associated with higher blood pressure in 4-year-old IVF offspring? Part I: multivariable regression analysis. Hum Reprod 2014; 29:502-509.

Selbing A, Kjessler B. Conceptual dating by ultrasonic measurement of the fetal biparietal diameter in early pregnancy. Acta Obstet Gynecol Scand 1985; 64:593-597.

Shaw L, Sneddon SF, Zeef L, Kimber SJ, Brison DR. Global gene expression profiling of individual human oocytes and embryos demonstrates heterogeneity in early development. PloS one 2013; 8:e64192.

Shioda Y, Tsunoda Y. Effect of storage period of the culture medium (M16) on the development of mouse zygotes in vitro and in vivo. J Mamm Ova Res 1986; 3:91-96.

Sinawat S, Hsaio WC, Flockhart JH, Kaufman MH, Keith J, West JD. Fetal abnormalities produced after preimplantation exposure of mouse embryos to ammonium chloride. Hum Reprod 2003; 18:2157-2165.

Sjoblom C, Roberts CT, Wikland M, Robertson SA. Granulocyte-macrophage colony-stimulating factor alleviates adverse consequences of embryo culture on fetal growth trajectory and placental morphogenesis. Endocrinology 2005; 146:2142-2153.

Smith GD, Takayama S, Swain JE. Rethinking in vitro embryo culture: new developments in culture platforms and potential to improve assisted reproductive technologies. Biol Reprod 2012; 86:62.

Smyth GK. Limma: linear models for microarray data Bioinformatics and computational biology solutions using R and Bioconductor. 2005. Springer, New York, pp. 397-420.

Steegers-Theunissen RP, Twigt J, Pestinger V, Sinclair KD. The periconceptional period, reproduction and long-term health of offspring: the importance of one-carbon metabolism. Hum Reprod Update 2013; 19:640-655.

Steptoe PC, Edwards RG. Birth after the reimplantation of a human embryo. Lancet 1978; 2:366.

Steuerwald NM, Bermudez MG, Wells D, Munne S, Cohen J. Maternal age-related differential global expression profiles observed in human oocytes. Reprod Biomed Online 2007; 14:700-708.

Stewart-Savage J, Bavister BD. Deterioration of stored culture media as monitored by a sperm motility bioassay. J In Vitro Fert Embryo Transf 1988; 5:76-80.

Summers MC, Biggers JD. Chemically defined media and the culture of mammalian preimplantation embryos: historical perspective and current issues. Hum Reprod Update 2003; 9:557-582.

Summers MC, McGinnis LK, Lawitts JA, Biggers JD. Mouse embryo development following IVF in media containing either L-glutamine or glycyl-L-glutamine. Hum Reprod 2005; 20:13641371.

Summers MC, McGinnis LK, Lawitts JA, Raffin M, Biggers JD. IVF of mouse ova in a simplex optimized medium supplemented with amino acids. Hum Reprod 2000; 15:1791-1801.

Sunde A, Balaban B. The assisted reproductive technology laboratory: toward evidence-based practice? Fertil Steril 2013; 100:310-318. 
Talebi A, Zavareh S, Kashani MH, Lashgarbluki T, Karimi I. The effect of alpha lipoic acid on the developmental competence of mouse isolated preantral follicles. J Assist Reprod Genet 2012; 29:175-183.

Tareq K, Miah AG, Salma U, Yoshida M, Tsujii H. Effect of amino acids and dipeptides on accumulation of ammonia in the medium during in vitro maturation and fertilization of porcine oocytes. Reprod Med Biol 2007; 6:165-170.

Thompson JG, Gardner DK, Pugh PA, McMillan WH, Tervit HR. Lamb birth weight is affected by culture system utilized during in vitro pre-elongation development of ovine embryos. Biol Reprod 1995; 53:1385-1391.

TNO, Dutch Organisation for Applied Scientific Research. 2010. TNO Groeicalculator: http://groeiweb.pgdata.nl/calculator.asp

Utsunomiya T, Naitou T, Nagaki M. A prospective trial of blastocyst culture and transfer. Hum Reprod 2002; 17:1846-1851.

Valenzuela-Alcaraz B, Crispi F, Bijnens B, Cruz-Lemini M, Creus M, Sitges M, Bartrons J, Civico S, Balasch J, Gratacos E. Assisted reproductive technologies are associated with cardiovascular remodeling in utero that persists postnatally. Circulation 2013; 128:14421450.

van lersel MP, Kelder T, Pico AR, Hanspers K, Coort S, Conklin BR, Evelo C. Presenting and exploring biological pathways with PathVisio. BMC Bioinformatics 2008; 9:399.

van Montfoort AP, Fiddelers AA, Janssen JM, Derhaag JG, Dirksen CD, Dunselman GA, Land JA, Geraedts JP, Evers JL, Dumoulin JC. In unselected patients, elective single embryo transfer prevents all multiples, but results in significantly lower pregnancy rates compared with double embryo transfer: a randomized controlled trial. Hum Reprod 2006; 21:338-343.

van Montfoort AP, Hanssen LL, de Sutter P, Viville S, Geraedts JP, de Boer P. Assisted reproduction treatment and epigenetic inheritance. Hum Reprod Update 2012; 18:171-197.

Vassena R, Boue S, Gonzalez-Roca E, Aran B, Auer H, Veiga A, Izpisua Belmonte JC. Waves of early transcriptional activation and pluripotency program initiation during human preimplantation development. Development 2011; 138:3699-3709.

Vergouw CG, Kostelijk EH, Doejaaren E, Hompes PG, Lambalk CB, Schats R. The influence of the type of embryo culture medium on neonatal birth weight after single embryo transfer in IVF. Hum Reprod 2012; 27:2619-2626.

Vilska S, Tiitinen A, Hyden-Granskog C, Hovatta O. Elective transfer of one embryo results in an acceptable pregnancy rate and eliminates the risk of multiple birth. Hum Reprod 1999; 14:2392-2395.

Virant-Klun I, Tomazevic T, Vrtacnik-Bokal E, Vogler A, Krsnik M, Meden-Vrtovec H. Increased ammonium in culture medium reduces the development of human embryos to the blastocyst stage. Fertil Steril 2006; 85:526-528.

Wales RG, Whittingham DG. Decomposition of sodium pyruvate in culture media stored at 5 degrees $\mathrm{C}$ and its effects on the development of the preimplantation mouse embryo. J Reprod Fertil 1971; 24:126.

Wang H, Rodriguez-Osorio N, Feugang JM, Jung S-Y, Garrison K, Wolgemuth C, Greer L, Crenshaw M, Memili E. Effects of culture media and inhibitors on biology of porcine early embryonic development in vitro. Livest Sci 2009; 121:102-107.

Wang HM, Zhang X, Qian D, Lin HY, Li QL, Liu DL, Liu GY, Yu XD, Zhu C. Effect of ubiquitin-proteasome pathway on mouse blastocyst implantation and expression of matrix metalloproteinases-2 and -9. Biol Reprod 2004; 70:481-487.

Wang J, Norman R, Kristiansson P. The effect of various infertility treatments on the risk of preterm birth. Hum Reprod 2002; 17:945-949.

Watkins AJ, Fleming TP. Blastocyst environment and its influence on offspring cardiovascular health: the heart of the matter. J Anat 2009; 215:52-59. 
Watkins AJ, Lucas ES, Torrens C, Cleal JK, Green L, Osmond C, Eckert JJ, Gray WP, Hanson MA, Fleming TP. Maternal low-protein diet during mouse pre-implantation development induces vascular dysfunction and altered renin-angiotensin-system homeostasis in the offspring. Br J Nutr 2010; 103:1762-1770.

Watkins AJ, Lucas ES, Wilkins A, Cagampang FR, Fleming TP. Maternal periconceptional and gestational low protein diet affects mouse offspring growth, cardiovascular and adipose phenotype at 1 year of age. PloS one 2011; 6:e28745.

Watkins AJ, Platt D, Papenbrock T, Wilkins A, Eckert JJ, Kwong WY, Osmond C, Hanson M, Fleming TP. Mouse embryo culture induces changes in postnatal phenotype including raised systolic blood pressure. Proc Natl Acad Sci U S A 2007; 104:5449-5454.

Watkins AJ, Ursell E, Panton R, Papenbrock T, Hollis L, Cunningham C, Wilkins A, Perry VH, Sheth B, Kwong WY et al. Adaptive responses by mouse early embryos to maternal diet protect fetal growth but predispose to adult onset disease. Biol Reprod 2008; 78:299-306.

Weathersbee PS, Francis MM, Macaso TM, Sauer MV, Paulson RJ. A new long shelf life formulation of modified Ham's F-10 medium: biochemical and clinical evaluation. J Assist Reprod Genet 1995; 12:175-179.

Whitten WK. Culture of tubal mouse ova. Nature 1956; 177:96.

Whittingham DG. Culture of mouse ova. J Reprod Fertil Suppl 1971; 14:7-21.

Woldringh GH, Hendriks JC, van Klingeren J, van Buuren S, Kollee LA, Zielhuis GA, Kremer JA. Weight of in vitro fertilization and intracytoplasmic sperm injection singletons in early childhood. Fertil Steril 2011; 95:2775-2777.

Wunder D, Ballabeni P, Roth-Kleiner M, Primi MP, Senn A, Chanson A, Germond M, Leyvraz C. Effect of embryo culture media on birth weight and length in singleton term infants after IVFICSI. Swiss Med Wkly 2014; 144:w14038.

Xiao $Y$, Isaacs SN. Enzyme-linked immunosorbent assay (ELISA) and blocking with bovine serum albumin (BSA)--not all BSAs are alike. J Immunol Methods 2012; 384:148-151.

Xue Z, Huang K, Cai C, Cai L, Jiang CY, Feng Y, Liu Z, Zeng Q, Cheng L, Sun YE et al. Genetic programs in human and mouse early embryos revealed by single-cell RNA sequencing. Nature 2013; 500:593-597.

Yan L, Yang M, Guo H, Yang L, Wu J, Li R, Liu P, Lian Y, Zheng X, Yan J et al. Single-cell RNA-Seq profiling of human preimplantation embryos and embryonic stem cells. Nat Struct Mol Biol 2013; 20:1131-1139.

Yin TL, Zhang Y, Li SJ, Zhao M, Ding JL, Xu WM, Yang J. Culture media influenced laboratory outcomes but not neonatal birth weight in assisted reproductive technology. J Huazhong Univ Sci Technolog Med Sci 2015; 35:932-937.

Young LE, Sinclair KD, Wilmut I. Large offspring syndrome in cattle and sheep. Rev Reprod 1998; 3:155-163.

Youssef MM, Mantikou E, van Wely M, Van der Veen F, Al-Inany HG, Repping S, Mastenbroek S. Culture media for human pre-implantation embryos in assisted reproductive technology cycles. Cochrane Database Syst Rev 2015; 11:CD007876.

Yuan Y, Krisher RL. Effect of ammonium during in vitro maturation on oocyte nuclear maturation and subsequent embryonic development in pigs. Anim Reprod Sci 2010; 117:302-307.

Zander DL, Thompson JG, Lane M. Perturbations in mouse embryo development and viability caused by ammonium are more severe after exposure at the cleavage stages. Biol Reprod 2006; 74:288-294.

Zandstra H, Van Montfoort AP, Dumoulin JC. Does the type of culture medium used influence birth weight of children born after IVF? Hum Reprod 2015; 30:530-542.

Zeger SL, Liang KY. Longitudinal data analysis for discrete and continuous outcomes. Biometrics 1986; 42:121-130. 
Zhu J, Li M, Chen L, Liu P, Qiao J. The protein source in embryo culture media influences birth weight: a comparative study between G1 v5 and G1-PLUS v5. Hum Reprod 2014a; 29:13871392.

Zhu J, Lin S, Li M, Chen L, Lian Y, Liu P, Qiao J. Effect of in vitro culture period on birth weight of singleton newborns. Hum Reprod 2014b; 29:448-454.

Ziebe S, Loft A, Povlsen BB, Erb K, Agerholm I, Aasted M, Gabrielsen A, Hnida C, Zobel DP, Munding B et al. A randomized clinical trial to evaluate the effect of granulocyte-macrophage colonystimulating factor (GM-CSF) in embryo culture medium for in vitro fertilization. Fertil Steril 2013; 99:1600-1609. 
Valorization 


\section{Valorization}

In this Valorization paragraph the potential impact of the research described in this thesis is considered.

\section{Relevance}

During the last decade, the use of ART has increased significantly worldwide and thereby the number of children born after ART too. The etiology of the adverse perinatal outcome of ART children is still incompletely understood. The extreme differences in birth weight that have been observed in animal studies, such as the large offspring syndrome, have not been observed in humans. However, ART children may be predisposed for adult health risks. With the growing number of children conceived after ART, it is important that the safety of ART is investigated. We have shown that culture media have a significant effect on fetal development, birth weight and early postnatal weight. Moreover, the gene expression in human preimplantation embryos is affected by the type of culture medium used.

ART has turned into a multi-million dollar industry and in most countries it is mainly provided in the private health care sector. Delivery rates are an important factor for IVF clinics, as IVF patients use these for choosing their clinic of treatment. This could increase the pressure on IVF clinics to use the latest technology in hope to achieve the best results. New technologies might get introduced based on expected benefits, which might not have been proven by randomized controlled trials. In ART there are several examples of new technologies and methods which have been introduced in clinical practice without appropriate evidence-based practice showing that the procedure is safe and beneficial to the patient, that it is cost-effective, and that its benefits outweigh its potential harms. Examples are assisted hatching, blastocyst transfer, endometrial 'scratching', in vitro maturation, time-lapse monitoring of embryo development and vitrification. Furthermore, changes in culture media composition, stimulation regimens and laboratory protocols are often established worldwide without adequate validation.

\section{Target groups}

The results of this thesis are interesting for IVF professionals, culture medium manufacturers, health economists and health care providers/insurers, politicians and the European Society of Human Reproduction and Embryology (ESHRE). Furthermore, our research results are of interest to couples who are planning to undergo an IVF treatment. Are they aware that they might receive treatments that are not validated by RCTs and are they aware of the adverse outcome after 
ART or of the possible long-term health effects? Couples undergoing an IVF treatment belong to a very vulnerable group, as they will do everything to get their own baby.

\section{Activities/innovation}

All papers have been published in a high-ranking scientific research journal. We have discussed our findings at national and international conferences to gain more attention for this topic. This has led to more research groups worldwide investigating the effect of culture medium and other ART related factors on human perinatal outcome. Currently, in our center we are involved in a study that investigates the effect of culture medium on the health of IVF children at the age of 9 years, with the focus on physiological (blood pressure and endothelial function), metabolic (lipid profile and glucose), and anthropometric (length, weight and skin fold thickness) examinations. Recently, in the Netherlands a new multicenter RCT started to compare pregnancy and perinatal outcome between the sequential culture medium Vitrolife G5 and Irvine's single step Continuous Single Culture medium. The ESHRE appointed a working group on culture media with members from the special interest groups Embryology, Safety and Quality in ART, and Genetics. This working group encourages constructive co-operation with IVF culture media manufacturers over transparency, composition and quality control parameters. Recently, Oxford University Press published a press release together with the RCT from this thesis and a review from the working group, which received worldwide attention.

The possibility that IVF culture media and other culture conditions are partly responsible for an adverse perinatal outcome in IVF children should not be ignored. However, the extend of the adverse outcome is still uncertain and large randomized studies are required to investigate the etiology of the adverse perinatal outcome. In addition, the full composition of embryo culture media should be made publically available by the companies that produce them. Companies should also report what studies have been performed to test these media and which endpoints have been analyzed.

\section{Schedule and implementation}

Before introducing changes to culture media composition, stimulation regimens and laboratory protocols into the clinical setting, IVF professionals need to consider their safety. New technologies should be evaluated for safety, effectiveness and cost-effectiveness. The use of the mouse embryo assay for preclinical testing should be discussed and the need for a more appropriate test should be recognized. Subsequently, human embryos should be made available 
for research, and research should be performed on human eggs/sperm or embryos donated for research. Large clinical trials with follow-up of IVF pregnancies and children should become the standard. 
Summary 


\section{Summary}

Chapter 1 provides a general introduction and the outline of this thesis. Children born after assisted reproductive technology (ART) are at increased risk of adverse perinatal outcomes such as preterm birth (PTB) and low birth weight (LBW) when compared with naturally conceived children. The adverse outcome in IVF children may be related to patient-related factors such as the age of the mother and the infertility of the parents, and to specific aspects of the ART technique such as the hormonal stimulation and culture conditions. From animal studies, it is clear that culture medium constituents can have profound effects on the perinatal outcome of the offspring, however, human studies are limited so far. This thesis focuses on the effect of embryo culture media on perinatal and postnatal outcome of ART children. Furthermore, this thesis assesses which components of the culture media might play an important role in this, and what the mode of action may be.

In Chapter 2, we evaluated the effect of in vitro culture medium on pregnancy and perinatal outcome by conducting a multicenter, double-blind randomized controlled trial comparing the use of HTF and G5 embryo culture media in IVF. Between July 2010 and May 2012, 836 couples (419 in the HTF group and 417 in the G5 group) were included. The live birth rate was higher, albeit nonsignificantly, in couples assigned to G5 than in couples assigned to HTF (44.1\% (184/417) vs. $37.9 \%$ (159/419); RR: 1.2 ; $95 \%$ Cl: 0.99 to 1.37). Number of utilizable embryos, implantation rate and clinical pregnancy rate were significantly higher for couples assigned to G5. Of the 383 live born children in this trial, birth weight data from 380 children (300 singletons (G5: 163, HTF: 137) and 80 twin children (G5: 38, HTF: 42)) were retrieved. Birth weight was significantly lower in the G5 group compared with the HTF group, with a mean difference of $158 \mathrm{~g}(95 \% \mathrm{Cl}: 42-$ $275 \mathrm{~g} ; \mathrm{P}=0.008)$. More singletons were born preterm in the G5 group (8.6\% $(14 / 163)$ vs. $2.2 \%$ (3/137), but singleton birth weight adjusted for gestational age and gender ( $z$-score) was also lower in the $G 5$ than HTF group $(-0.13 \pm 0.08$ vs. $0.17 \pm 0.08 ; P=0.008)$. Our findings indicate that embryo culture media used in IVF not only affect treatment efficacy but also the perinatal outcome. This suggests that the millions of human embryos that are cultured in vitro each year are sensitive to their environment.

To study the effect of embryo culture medium on postnatal growth, we investigated if the difference in weight of singletons at birth between two different culture media groups (Cook and Vitrolife G3) would disappear, persist or increase postnatally in Chapter 3. From July 2003 to December 2006, a total of 1432 IVF treatment cycles with fresh embryo transfer were randomly allocated to have all embryos cultured in Vitrolife G3 medium $(n=715)$ or Cook medium $(n=$ 717). Two years after delivery, questionnaires were sent to the parents of all 
children to collect data about weight, height and head circumference around 1, 2, $3,4,6,7.5,9,11,14,18$, and 24 months of age. From the 294 live born singletons that fulfilled our inclusion criteria, 29 were lost to follow-up. The remaining 265 singletons (Cook group: 117, Vitrolife group: 148) were included in the analysis. Singletons in the Vitrolife group were heavier during the first 2 years of life compared with singletons in the Cook group. Cross-sectional analyses showed that adjusted weight SDS differed between groups at $1(0.35 \pm 0.14, P=0.010), 2$ (0.39 $\pm 0.14, P=0.006), 3(0.35 \pm 0.14, P=0.011), 4(0.30 \pm 0.13, P=0.020), 11$ $(0.28 \pm 0.13, P=0.036), 14(0.32 \pm 0.13, P=0.014)$, and $24(0.39 \pm 0.15, P=0.011)$ months of age, while adjusted height SDS was only significantly different at 1 $(0.21 \pm 0.11, P=0.048)$ month of age. Head circumference was similar between the two groups at all ages. Longitudinal analyses showed that both postnatal weight $(P=0.005)$ and height $(P=0.031)$ differed between the groups throughout the first 2 years of life, while the growth velocity was not significantly different between the two groups. This study indicates that the effect of culture medium during the first few days after fertilization on prenatal growth and birth weight persists at least during the first 2 years of life.

In Chapter 4, we investigated the effect of age of Vitrolife G5 embryo culture medium on embryo development, ongoing pregnancy and birth weight of the newborns. Data from 1832 IVF/ICSI cycles with fresh embryo transfer, performed in the period 2008-2012 was used. Age of the culture medium was not associated with fertilization rate $(P=0.543)$, early cleavage rate $(P=0.155)$, percentage of embryos containing four or more cells on Day $2(P=0.401)$, percentage of embryos containing eight or more cells on Day $3(P=0.175)$, percentage of embryos with multinucleated blastomeres $(P=0.527)$, or ongoing pregnancy $(P=$ 0.729). However, birth weight of the newborn was inversely associated with age of the medium ( $\beta=-3.6 \mathrm{~g}, \mathrm{SE}: 1.5 \mathrm{~g}, \mathrm{P}=0.021)$, after controlling for possible confounders (day of embryo transfer, number of transferred embryos, child's gender, gestational age at birth, parity, pregnancy complications, maternal smoking, height and weight, and paternal height and weight) and the association was not biased by year of treatment, time since first opening of the bottle or batch variations. This indicates a difference of 234 grams in birth weight of newborns for media with an age difference of 65 days. It is not unlikely that culture media lose their ability to properly support embryo development over time by deterioration of components.

One example of deterioration is the spontaneous break down of amino acids into ammonium (as one of the substances produced) during incubation at $37^{\circ} \mathrm{C}$. Ammonium has been found to be detrimental for embryo development, therefore, we evaluated the ammonium accumulation during storage at $2-8^{\circ} \mathrm{C}$ and incubation at $37^{\circ} \mathrm{C}$ in commercially available culture media and protein 
supplements used for in vitro development of human preimplantation embryos (Chapter 5). Ammonium accumulation was investigated in 15 ready-to-use media, 11 protein-free media and 8 protein supplements. All ready-to-use, i.e. protein pre-supplemented, culture media showed ammonium accumulation during storage for 6 weeks (ranging from 9.2 to $99.8 \mu \mathrm{M}$ ) and during incubation for 4 days (ranging from 8.4 to $138.6 \mu \mathrm{M}$ ), resulting in levels that might affect embryo development. The protein supplements also showed ammonium accumulation, while the culture media without protein supplementation did not. The main sources of ammonium buildup in ready-to-use culture media were unstable glutamine and the protein supplements. No additional ammonium buildup was found during incubation when using an oil overlay or with the presence of an embryo in the culture droplet. Breakdown of components into ammonium is more pronounced during incubation at $37^{\circ} \mathrm{C}$, however, it is not negligible during storage at $2-8^{\circ} \mathrm{C}$. This results in increasing ammonium levels in culture media over time that may affect embryo development.

To explore the effect of embryo culture medium (G5 and HTF) on the epigenetic regulation in human preimplantation embryos, in Chapter 6 we carried out a microarray analysis ( $\mathrm{n}=10$ in each group) to investigate genome-wide which pathways are differently affected by the culture media. Expression of 951 genes differed significantly $(P<0.01)$ between the $G 5$ and HTF groups. Eighteen pathways, involved in apoptosis, metabolism, protein processing and cell-cycle regulation, showed a significant overrepresentation of differentially expressed genes. The DNA replication, $G 1$ to $S$ cell-cycle control and oxidative phosphorylation pathways were upregulated in the G5 group compared with the HTF group. This is in agreement with the morphological assessment of the 1527 embryos (originating from 2PN zygotes), which showed that embryos consisted of more cells on Day $2(3.73 \pm 1.30$ versus $3.40 \pm 1.35, \mathrm{P}<0.001)$ and Day $3(7.00 \pm$ 2.41 versus $5.84 \pm 2.36, \mathrm{P}<0.001$ ) in the $\mathrm{G} 5$ group when compared with the HTF group. Furthermore, the implantation rate was significantly higher in the $\mathrm{G} 5$ group compared with the HTF group ( $26.7 \%$ versus $14.7 \%, P=0.002)$ after transfer on the second or the third day after fertilization. This study shows that gene expression in human preimplantation embryos is altered by the culture medium used during IVF treatment and provides insight into the biological pathways that are affected.

Chapter 7 presents a discussion of the findings of this thesis. In short, the studies in this thesis, together with other studies, have shown that ART-procedure related factors such as embryo culture media - next to subfertility itself - play an important role in the adverse perinatal outcome in ART singletons. It is unknown what the long-term health effects will be and long-term follow-up studies should be conducted to evaluate the health of ART offspring worldwide. 
Samenvatting 


\section{Samenvatting}

Hoofdstuk 1 geeft een algemene inleiding en een overzicht van dit proefschrift. Kinderen verwekt via geassisteerde voortplanting (ART) hebben een slechtere perinatale uitkomst, zoals vroeggeboorte en laag geboortegewicht, in vergelijking met kinderen geboren na natuurlijke verwekking. De slechtere perinatale uitkomst bij IVF kinderen kan komen door patiënt-gerelateerde factoren en specifieke aspecten van de ART techniek. Uit dierstudies is duidelijk gebleken dat de perinatale uitkomst van nakomelingen mede bepaald wordt door het kweekmedium, echter is dit verband bij de mens nog onduidelijk. Dit proefschrift richt zich op het effect van embryo kweekmedia op de perinatale en postnatale uitkomst van ART kinderen. Daarnaast wordt in dit proefschrift bekeken welke componenten van de kweekmedia hierbij een belangrijke rol kunnen spelen en via welk mechanisme dit verloopt.

In Hoofdstuk 2 evalueren we het effect van in vitro kweekmedium op zwangerschaps- en perinatale uitkomsten via een multicenter, dubbel geblindeerde, gerandomiseerde studie, waarbij het gebruik van G5 medium en HTF medium wordt vergeleken. Van juli 2010 tot en met mei 2012 werden 836 koppels (419 in de HTF groep en 417 in de G5 groep) geïncludeerd. De proportie levendgeborenen was hoger, echter niet significant, voor koppels uit de G5 groep in vergelijking met koppels uit de HTF groep (44.1\% (184/417) versus $37.9 \%$ (159/419); RR: $1.2 ; 95 \% \mathrm{Cl}: 0.99$ tot 1.37). Het aantal te gebruiken embryo's, de implantatie ratio en klinische zwangerschapskansen waren significant hoger voor koppels uit de G5 groep. Van de 383 levend geboren kinderen in deze studie, is het geboortegewicht van 380 kinderen (300 eenlingen (G5: 163, HTF: 137) en 80 tweelingen (G5: 38, HTF: 42)) geanalyseerd. Het geboortegewicht was significant lager in de G5 groep in vergelijking met de HTF groep, met een gemiddeld verschil van $158 \mathrm{~g}$ (95\% Cl: 42 tot $275 \mathrm{~g} ; \mathrm{P}=0.008)$. In de $\mathrm{G} 5$ groep waren er meer vroeggeboortes $(8.6 \%(14 / 163)$ versus $2.2 \%$ (3/137), echter was ook het geboortegewicht voor eenlingen na correctie voor zwangerschapsduur en geslacht (Z-score) lager in de G5 groep ten opzichte van de HTF groep (-0.13 \pm 0.08 versus $0.17 \pm 0.08 ; P=0.008$ ). Onze bevindingen geven aan dat embryo kweekmedia zowel de efficiëntie van de behandeling als de perinatale uitkomst beïnvloeden. Dit zou betekenen dat de miljoenen menselijke embryo's die jaarlijks gekweekt worden, gevoelig zijn voor hun omgeving.

Om het effect van embryo kweekmedia op de postnatale groei te onderzoeken, hebben we in Hoofdstuk 3 gekeken of het verschil in gewicht van eenlingen (uit een voorgaande studie), dat was ontstaan na embryokweek in Cook of Vitrolife G3, zou verdwijnen, aanhouden of toenemen gedurende de eerste twee levensjaren. Van juli 2003 tot en met december 2006 werd bij 1432 IVF cycli met 
verse embryo terugplaatsing afwisselend gebruik gemaakt van Cook medium ( $\mathrm{n}=$ 717) of Vitrolife G3 medium ( $n=715$ ). Twee jaar na de geboorte van de kinderen werden naar de ouders vragenlijsten gestuurd om de gegevens over het gewicht, lengte en hoofdomtrek van de kinderen op te vragen aan de hand van het Groeiboekje van het consultatiebureau. Van de 294 kinderen die in deze studie geïncludeerd waren, konden van 29 kinderen geen gegevens verzameld worden. De gegevens van de overgebleven 265 kinderen (Cook: 117, Vitrolife: 148) werden voor de analyse gebruikt. Kinderen uit de Vitrolife groep waren gedurende de eerste twee levensjaren zwaarder dan kinderen in de Cook groep. Crosssectionele analyses toonden een verschil in gecorrigeerd SDS gewicht bij $1(0.35 \pm$ $0.14, P=0.010), 2(0.39 \pm 0.14, P=0.006), 3(0.35 \pm 0.14, P=0.011), 4(0.30 \pm$ $0.13, P=0.020), 11(0.28 \pm 0.13, P=0.036), 14(0.32 \pm 0.13, P=0.014)$ en 24 (0.39 $\pm 0.15, P=0.011$ ) maanden, terwijl de gecorrigeerde SDS lengte alleen verschilde bij 1 maand $(0.21 \pm 0.11, P=0.048)$. Hoofdomtrek was gelijk tussen beide groepen op alle tijdstippen. Longitudinale analyses toonden een verschil in postnataal gewicht $(P=0.005)$ en lengte $(P=0.031)$ gedurende de eerste twee levensjaren, terwijl de groeisnelheid niet significant verschilde tussen de groepen. Deze studie toont aan dat het effect van embryokweekmedium gedurende de eerste dagen na bevruchting op de prenatale groei aanhoudt, ten minste gedurende de eerste twee levensjaren.

In Hoofdstuk 4 onderzochten we het effect van de ouderdom van Vitrolife G5 embryo kweekmedium op embryonale ontwikkeling, zwangerschapskansen en het geboortegewicht van de pasgeborenen. Gegevens van 1832 IVF/ICSI cycli met verse embryo terugplaatsingen, uit de periode 2008 tot en met 2012, werden gebruikt. De ouderdom van het kweekmedium was niet geassocieerd met de kans op bevruchting $(P=0.543)$, kans op "early cleavage" $(P=0.155)$, het percentage embryo's dat uit vier of meer cellen bestond op Dag $2(P=0.401)$, het percentage embryo's dat uit 8 of meer cellen bestond op Dag $3(P=0.175)$, het percentage embryo's dat blastomeren met meerdere kernen bevatte $(P=0.527)$ en de kans op een doorgaande zwangerschap $(P=0.729)$. Het geboortegewicht van de pasgeborenen was omgekeerd geassocieerd met de ouderdom van het kweekmedium ( $\beta=-3.6 \mathrm{~g}, \mathrm{SE}: 1.5 \mathrm{~g}, \mathrm{P}=0.021$ ), na correctie voor mogelijke confounders (aantal dagen kweek, aantal teruggeplaatste embryo's, geslacht van de pasgeborene, zwangerschapsduur, pariteit, zwangerschapscomplicaties, rookgedrag van de moeder, lengte en gewicht van de ouders), en werd niet beïnvloed door jaar van behandeling, tijd tussen gebruik en openen van het flesje kweekmedium en variaties in batches. Dit duidt op een afname van $234 \mathrm{~g}$ in geboortegewicht bij medium dat 65 dagen ouder is. Embryo kweekmedia verliezen wellicht hun vermogen om de embryonale ontwikkeling te ondersteunen door afbraak van bepaalde ingrediënten. 
Een voorbeeld hiervan is de spontane afbraak van aminozuren, waarbij onder andere ammonium vrijkomt. Ammonium is slecht voor de ontwikkeling van het embryo en daarom hebben we in Hoofdstuk 5 de ammoniumtoename in commercieel verkrijgbare embryo kweekmedia en eiwit supplementen, gedurende opslag bij $2-8^{\circ} \mathrm{C}$ en gedurende incubatie bij $37^{\circ} \mathrm{C}$, onderzocht. In alle kant-en-klare, i.e. eiwit bevattende, kweekmedia was er ammoniumtoename gedurende opslag voor 6 weken (variërend tussen 9.2 en $99.8 \mu \mathrm{M}$ ) en gedurende incubatie voor 4 dagen (variërend tussen 8.4 en $138.6 \mu \mathrm{M}$ ), wat kan resulteren in ammoniumconcentraties welke de embryonale ontwikkeling negatief beïnvloeden. De eiwit supplementen vertoonden ook ammoniumtoename, terwijl de eiwit-vrije kweekmedia geen aantoonbare toename in ammonium lieten zien. De belangrijkste bronnen voor de ammoniumtoename in de kant-en-klare media waren het instabiele aminozuur glutamine en de eiwit supplementen. Er werd geen extra ammoniumtoename waargenomen tijdens incubatie als de kweekdruppels werden bedekt met minerale olie of bij aanwezigheid van een embryo in de druppel. Ondanks dat de toename van ammonium in kweekmedia door afbraak van bepaalde ingrediënten beduidend groter is tijdens incubatie bij $37^{\circ} \mathrm{C}$, is de ammoniumtoename tijdens opslag bij $2-8^{\circ} \mathrm{C}$ niet te verwaarlozen. Dit resulteert in toenemende ammonium waardes in de kweekmedia na verloop van tijd, wat de embryonale ontwikkeling negatief kan beïnvloeden.

Om het effect van kweekmedia (G5 en HTF) op de epigenetische regulatie in menselijke pre-implantatie embryo's te bekijken, hebben we in Hoofdstuk 6 een microarray analyse ( $n=10$ in elke groep) uitgevoerd om genoom breed te onderzoeken welke pathways verschillend waren tussen de twee groepen. Expressie van 951 genen verschilden significant $(P<0.01)$ tussen de $G 5$ en HTF groepen. Achttien pathways betrokken bij apoptose, metabolisme, eiwitverwerking en controle van de celcyclus, toonden een significante overrepresentatie van genen die verschillend tot expressie kwamen tussen de twee groepen. De DNA replicatie, G1 naar S celcyclus controle en oxidatieve fosforylering pathways waren up-gereguleerd in de G5 groep ten opzichte van de HTF groep. Dit is in overeenstemming met de morfologische scoring van 1527 embryo's, waaruit bleek dat embryo's uit de G5 groep uit meer cellen op Dag 2 $(3.73 \pm 1.30$ versus $3.40 \pm 1.35, P<0.001)$ en op Dag $3(7.00 \pm 2.41$ versus $5.84 \pm$ 2.36, $\mathrm{P}<0.001)$ bestaan ten opzichte van embryo's uit de HTF groep. Bovendien was de implantatie significant hoger in de G5 groep dan in de HTF groep $(26.7 \%$ versus $14.7 \%, \mathrm{P}=0.002$ ) bij terugplaatsingen op de tweede of derde dag na bevruchting. Deze studie toont aan dat de gen expressie in humane preimplantatie embryo's wordt beïnvloed door het kweekmedium dat wordt gebruikt tijdens de IVF behandeling. Daarbij verschaft het inzicht in de biologische pathways die beïnvloed worden. 
Hoofdstuk 7 geeft een discussie van de bevindingen uit dit proefschrift. Samenvattend, tonen de studies uit dit proefschrift, samen met studies van anderen, aan dat ART-gerelateerde factoren zoals embryo kweekmedia een belangrijke rol spelen bij de slechtere perinatale uitkomst in ART kinderen. Het is nog onbekend wat de langetermijneffecten zullen zijn en deze moeten onderzocht worden in follow-up studies om wereldwijd de gezondheid van ART nakomelingen te evalueren. 

Dankwoord 


\section{Dankwoord}

Promoveren is geen individuele bezigheid. Graag wil ik iedereen bedanken die heeft bijgedragen aan het succesvol afronden van mijn promotie-traject.

Prof. dr. Evers, beste Hans, bedankt dat u mij wilde begeleiden bij mijn promotietraject. Bij het schrijven van de artikelen kon ik altijd rekenen op een snelle reactie van $u$, waarbij uw waardevolle opmerkingen positief bijdroegen aan de kwaliteit van de artikelen. Ook was $u$ altijd aanwezig bij mijn presentaties tijdens de jaarlijkse ESHRE congressen, ondanks dat u deze al herhaaldelijk had gehoord tijdens de voorbereidingen.

Geachte dr. Dumoulin, beste John, bedankt voor het mooie promotie-traject. Jouw artikel uit 2010 vormde de basis voor de onderzoeken uit dit proefschrift. Ik ben blij dat Aafke en jij mij de kans hebben gegeven om deze onderzoeken uit te voeren. Een enorme waardering heb ik voor jouw 'dBase' (waar afgelopen jaar afscheid van is genomen bij het ingebruikname gaan van een nieuw systeem). Succes in het nieuwe lab!

Geachte dr. van Montfoort, beste Aafke, toen ik bij jou op gesprek kwam voor een stageplek, leerde ik direct jouw enthousiasme en positieve instelling kennen. Wij vormden tijdens mijn stages en promotie-traject het onderzoeksteam binnen het IVF-lab. Het was geweldig om met jou samen te zitten om voor een bepaald vraagstuk tot een oplossing te komen. Ik waardeer jouw kennis van het onderzoek en van de verschillende technieken waarmee we gewerkt hebben. Ik kon altijd bij jou terecht en heb veel van je geleerd. Ontzettend bedankt!

Mijn collega's van het IVF-lab (naast Aafke en John), Danielle, Edith, Edith, Inge, Josien, Marij, Marijke, Marion, Myriam en Sabina. Bedankt voor de gezellige en leerzame tijd! Naast jullie reguliere werkzaamheden waren jullie altijd bereid om iets voor het onderzoek te doen. Het was fantastisch om deel uit te maken van zo'n hecht team.

De overige collega's van de voortplantingsgeneeskunde; Carla, Celine, Danielle, Desiree, Erna, Gerard, Germaine, Guusje, Heleen, Janneke, Katinka, Nienke, Ricky, Ron, Sylvia, Tonny en Trudy. Bedankt voor de gezellige tijd, er was altijd wat te lachen met jullie. Bedankt voor de waardevolle inbreng bij de wetenschappelijke besprekingen.

Graag bedank ik alle medeauteurs voor hun bijdrage aan de onderzoeken en publicaties: Aafke, Alex, Dimitri, Edith, Eleni, Els, Hans, Inge, Jannie, John, Josien, Lars, Lobke, Luc, Madelon, Martijs, Otto, Sebastiaan, Sjoerd, Tessa en Wolfgang. 
Dank aan alle onderzoeksmedewerkers, gynaecologen en verloskundigen voor het opzoeken en aanleveren van de data voor de RCT.

Geachte leden van de beoordelingscommissie, ik dank $u$ hartelijk voor het lezen en beoordelen van het proefschrift. (Dear members of the assessment committee, thank you for the assessment of my PhD thesis.)

Dank aan alle vrouwen en hun partners die hebben deelgenomen aan de onderzoeken uit dit proefschrift. Ik hoop dat jullie droom is uitgekomen!

Mijn vriendengroep (grotendeels) van de middelbare school: Ivo, Joost, Marco, René, Ron, Sander, Sander en Tom. Het is altijd weer een gezellige boel als we allemaal samen zijn. Joost en Natasja, fijn dat onze vriendschap nog steeds hecht is, ondanks de grote afstand. Bedankt voor jullie gastvrijheid, iedere keer weer, als wij jullie in Zwitserland komen opzoeken.

Math en Tineke, bedankt dat jullie altijd voor ons klaar staan en voor de oprechte interesse die jullie immer hebben getoond.

Stefan, Remko en Meta, wat hebben we samen een mooie jeugd gehad. Bedankt!

Pap en mam, ik ben ontzettend dankbaar voor jullie onvoorwaardelijke liefde en steun! Zowel vroeger als nu. Bedankt voor de mooie jeugd die wij aan jullie te danken hebben. Ik hoop dat Caroline en ik onze kinderen ook zo'n goede basis kunnen geven.

Lieve Feline, Rosalie en Casper, mijn schatjes! Wat is het een genot om jullie te zien opgroeien! Mama en ik hebben geprobeerd om jullie een goede start te geven (denk onder andere aan de epigenetische regulatie tijdens jullie vroege bestaan) en ik hoop dat we jullie een geweldige toekomst kunnen geven!

Lieve Caroline, wat ben ik ontzettend blij met jou! Wat zorg jij goed voor mij en de kinderen! Wat boffen wij toch met jou! Ik ben enorm trots op jou en ik hou heel veel van jou. Bedankt voor alles! 

Curriculum Vitae 


\section{Curriculum Vitae}

Sander Kleijkers werd op 15 juli 1982 te Heerlen geboren. In 2000 behaalde hij het VWO-diploma aan Scholengemeenschap Trevianum (locatie Serviam) te Sittard. Vervolgens begon hij met de studie Psychologie aan de Universiteit Maastricht. Twee jaar later stapte hij over naar de studie Moleculaire Levenswetenschappen (MLW)/Biomedische Wetenschappen aan de transnationale Universiteit Limburg (een samenwerking tussen Universiteit Maastricht en Universiteit Hasselt). In de laatste twee jaren van zijn opleiding, liep hij stages bij het IVF-lab van het Maastricht UMC+. Na het behalen van zijn masterdiploma MLW is hij in 2010 begonnen als promovendus binnen het IVF-lab, waar hij het in dit proefschrift beschreven onderzoek heeft uitgevoerd.

Sander is getrouwd met Caroline en zij hebben drie kinderen: Feline, Rosalie en Casper.

\section{Publicaties}

Kleijkers SHM, Mantikou E, Slappendel E, Consten D, van Echten-Arends J, Wetzels AM, van Wely M, Smits LJM, van Montfoort APA, Repping S, Dumoulin JCM, Mastenbroek S. Influence of embryo culture medium (G5 and HTF) on pregnancy and perinatal outcome after IVF: a multicenter RCT. Human Reproduction 2016, in press.

Kleijkers SHM, van Montfoort APA, Bekers O, Coonen E, Derhaag JG, Evers JLH, Dumoulin JCM. Ammonium accumulation in commercially available embryo culture media and protein supplements during storage at $2-8^{\circ} \mathrm{C}$ and during incubation at $37^{\circ} \mathrm{C}$. Human Reproduction 2016;31(6):1192-1199.

Kleijkers SHM, Eijssen LMT, Coonen E, Derhaag JG, Mantikou E, Jonker MJ, Mastenbroek S, Repping S, Evers JLH, Dumoulin JCM, van Montfoort APA. Differences in gene expression profiles between human preimplantation embryos cultured in two different IVF culture media. Human Reproduction 2015;30(10):2303-2311.

Kleijkers SHM, van Montfoort APA, Smits LJM, Coonen E, Derhaag JG, Evers JLH, Dumoulin JCM. Age of G-1 PLUS v5 embryo culture medium is inversely associated with birth weight of the newborn. Human Reproduction 2015;30(6):1352-1357.

Kleijkers SHM, van Montfoort APA, Smits LM, Viechtbauer W, Roseboom TJ, Nelissen ECM, Coonen E, Derhaag JG, Bastings L, Schreurs IEL, Evers JLH, Dumoulin JCM. IVF culture medium affects post-natal weight in humans during the first 2 years of life. Human Reproduction 2014;29(4):661-669. 
